The Astrophysical Journal Supplement Series, 58:493-531, 1985 July

(1) 1985. The American Astronomical Society. All rights reserved. Printed in U.S.A.

\title{
THERMODYNAMICS OF CLATHRATE HYDRATE AT LOW AND HIGH PRESSURES WITH APPLICATION TO THE OUTER SOLAR SYSTEM ${ }^{1}$
}

\author{
JoNAThan I. LUNINE ${ }^{2}$ AND DAVID J. STEVENSON \\ Division of Geological and Planetary Sciences, California Institute of Technology \\ Received 1984 June 29; accepted 1985 January 10
}

\begin{abstract}
The thermodynamic stability of clathrate hydrate is calculated under a wide range of temperature and pressure conditions applicable to solar system problems, using a statistical mechanical theory developed by van der Waals and Platteeuw (1959) and existing experimental data on properties of clathrate hydrates and their components. At low pressure, dissociation pressures and partition functions (Langmuir constants) for CO clathrate (hydrate) have been predicted, using the properties of clathrate containing, as guests, molecules similar to $\mathrm{CO}$. The comparable or higher propensity of $\mathrm{CO}$ to incorporate in clathrate relative to $\mathrm{N}_{2}$ is used to argue for high CO-to- $\mathrm{N}_{2}$ ratios in primordial Titan if $\mathrm{N}_{2}$ was accreted as clathrate. The relative incorporation of noble gases in clathrate from a solar composition gas at low temperatures is calculated and applied to the case of giant-planet atmospheres and icy satellites. It is argued that nonsolar but well-constrained noble gas abundances will be measured by Galileo in the Jovian atmosphere if the observed carbon enhancement is due to bombardment of the atmosphere by clathrate-bearing planetesimals sometime after planetary formation. The noble gas abundances in Titan's atmosphere are also predicted under the hypothesis that much of the satellite's methane accreted as clathrate. Double occupancy of clathrate cages by $\mathrm{H}_{2}$ and $\mathrm{CH}_{4}$ in contact with a solar composition gas is examined, and it is concluded that potentially important amounts of $\mathrm{H}_{2}$ may have incorporated in satellites as clathrate. The kinetics of clathrate formation is also examined, and it is suggested that, under thermodynamically appropriate conditions, essentially complete clathration of water ice could have occurred in high-pressure nebulae around giant planets but probably not in the outer solar nebula; comets probably did not aggregate as clathrate. At moderate pressures, the phase diagram for methane clathrate hydrate in the presence of $15 \%$ ammonia (relative to water) is constructed, and application to the early Titan atmospheric composition is described. The high-pressure stability of $\mathrm{CH}_{4}, \mathrm{~N}_{2}$, and mixed $\mathrm{CH}_{4}-\mathrm{N}_{2}$ clathrate hydrate is calculated; conversion back to water and $\mathrm{CH}_{4}$ and/or $\mathrm{N}_{2}$ fluids or solids is predicted for pressures $\gtrsim 12$ kilobars (independent of temperature) and temperatures $\gtrsim 320 \mathrm{~K}$ (independent of pressure). The effect of ammonia is to shrink the $T-P$ stability field of clathrate with increasing ammonia concentration. These results imply that (1) clathrate is stable throughout the interior of Oberon- and Rhea-sized icy satellites, and (2) clathrate incorporated in the innermost icy regions of Titan would have decomposed, perhaps allowing buoyant methane to rise. Brief speculation on the implications of this conclusion for the origin of surficial methane on Titan is given. A list of suggested experiments and observations to test the theory and its predictions is presented.
\end{abstract}

Subject headings: molecular processes - planets: atmospheres - planets: satellites

\section{INTRODUCTION}

Clathrate hydrates are water-ice compounds in which a distinctive open lattice structure of the ice forms cages stabilized by the inclusion of molecules of other chemical species. As a means of incorporating large quantities of volatile gases in solid bodies under conditions well outside the stability field of the condensed phases of the volatiles, they have been of great interest in terrestrial and solar system studies. Increasing interest in the possible importance of clathrates in the outer solar system has arisen for two reasons. First, volatiles such as methane may owe their existence on Titan, Triton, and perhaps Pluto in part because of clathrates. Second, refined

\footnotetext{
${ }^{1}$ Contribution number 4068 of the Division of Geological and Planetary Sciences, California Institute of Technology, Pasadena, California.

${ }^{2}$ Now at Lunar and Planetary Laboratory, University of Arizona, Tucson, Arizona.
}

observational techniques have, for the first time, made direct observation of these compounds on outer solar system bodies a possibility, renewing interest in their thermodynamic properties.

In this paper we extend a statistical mechanical model of clathrate formation originally developed by van der Waals and Platteeuw (1959) to predict the formation conditions and composition of clathrate hydrate under a wide range of situations of interest to solar system studies. These include the low-pressure $\left(10^{-12}\right.$ to $\left.1 \mathrm{bar}\right)$ regimes of gaseous nebulae, in which the outer planets and their satellites may have formed, intermediate pressures ( 1 to $10^{2}$ bars) obtained in the presentday atmosphere of Titan and plausible models for primordial atmospheres, and high ( $\geq 10^{3}$ bars) pressure relevant to the interiors of large icy satellites. The goal of this paper is to treat all of these cases with a single, physically reasonable model of clathrate formation which is tied as much as possible directly 
to laboratory data on the stability of the various clathrate compounds or the thermodynamic properties of the pure components themselves. The model allows us to make a prediction for the conditions of formation of clathrate hydrate of $\mathrm{CO}$, an important molecule cosmochemically and one for which no laboratory data on the clathrate yet exist. We also predict substantial double occupancy of clathrate cages by $\mathrm{CH}_{4}$ and $\mathrm{H}_{2}$, the latter by itself being a poor clathrate hydrate former. This may have implications for satellite outgassing processes. We also deal, for the first time, with the stability of clathrates at high pressure and the formation of clathrates in the presence of an ammonia-water solution (a probable primordial environment in large icy satellites). The difficult problem of clathrate formation kinetics in a gaseous environment is also analyzed. We find that disequilibrium is likely in many circumstances, including the formation conditions of comets, but that approach to full equilibrium may occur if ice particles or planetesimals undergo extensive collisional gardening. Approach to equilibrium is more probable in the higher-density, higher-temperature nebulae around proto-giant planets than in the primordial solar nebula. A study of this scope has not been previously attempted in the literature; two areas for which few or no data exist, the high-pressure ( $>5$ kilobars) clathrate stability and formation of clathrate in the presence of ammonia-water solution, are dealt with for the first time here. Application to relevant solar system objects will be given to make the results more concrete; however, our results are intended to be incorporated into models for the properties and evolution of bodies for which additional considerations and constraints must be applied beyond the scope of the present paper. We are working on several of these applications; we hope, also, that the present effort will be useful to other workers in the field who desire to incorporate clathrate thermodynamics in their models.

The plan of the paper is as follows. Section II reviews pertinent literature on clathrate properties and occurrence as well as previous applications to solar system problems. Section III presents the statistical mechanical model used to predict clathrate formation. In § IV we calculate the phase diagram of clathrate hydrate in the presence of ammonia at moderate pressures. Section V uses the results given in $\S$ III to calculate the complete stability fields of both methane and molecular nitrogen clathrate hydrate up to tens of kilobars of pressure. Although almost no laboratory data are available at these pressures, the regime is directly relevant to the interiors of large satellites such as Titan and Triton, and perhaps the planet Pluto. Section VI addresses the problem of kinetics of clathrate formation. Section VII applies the results of previous sections to calculate the abundance of volatiles incorporated in clathrate formed from a solar composition gas and explores implications for the current composition and evolution of satellites and giant-planet atmospheres. Section VIII reviews our important results and lists the outstanding uncertainties in clathrate thermodymamics and kinetics which have yet to be resolved by experiment and theory.

Because the nomenclature used in the clathrate literature is confusing and not always consistent, we establish some conventions for the paper. We will deal almost exclusively with clathrate compounds for which water ice forms the lattice structure (i.e., the "host" molecule). Hence, the term clathrate is here understood to mean clathrate hydrate. The gas molecule occupying a cage site is the "guest" molecule. The term methane clathrate is used to denote a clathrate hydrate in which methane is the primary guest molecule. Occasional discussion of clathrates in which a substance other than water ice is the host, for example, quinol, will employ the term quinol clathrate, as in the literature, but care will be taken to be sufficiently explicit to avoid confusing the guest and host molecules in the minds of the readers. Finally, a stoichiometric compound of water ice, in which molecules bond chemically with the water molecule and do not form a clathrate, will be denoted as a hydrate, e.g., ammonia hydrate. Hence, the use of the term gas hydrate to denote a clathrate hydrate (employed in the geological and geophysical literature) is avoided here.

\section{BACKGROUND}

The history of synthesis of clathrate hydrate compounds extends back to the preparation of chlorine clathrate hydrate by Davy in 1811 and Faraday in 1823 (Jeffrey and McMullan 1967). The clathrate hydrate structure consists of open, cagelike voids, each formed by 20-28 hydrogen-bonded water molecules. Two structural types of clathrate hydrate are known, referred to as I and II; the size of the included guest molecule determines which is formed. Figure 1, from Miller (1973), illustrates the cage geometry of the structure I clathrate, first determined by Pauling and Marsh (1952), which is organized into cells with two small cages and six large. Structure II possesses cells with 16 small and 8 large cages, the latter about $10 \%$ bigger in radius than the structure I large cages. Data on the geometry of both types of clathrate hydrate structures are tabulated in Davidson (1971). Although previous workers have assumed that all molecules smaller than $5.8 \AA$ form structure I clathrate, very recent neutron diffraction results of Davidson et al. (1984) demonstrate that argon and krypton preferentially form structure II clathrate, while somewhat larger molecules such as $\mathrm{CH}_{4}$ form structure I.

Several good reviews of the literature up to the late sixties are Davidson (1973), Jeffrey and McMullan (1967), Miller (1974), Hagan (1962), and van der Waals and Platteeuw (1959). An interesting review from the Russian perspective is Byk and Fomina (1968). Of particular importance to the present study are the van der Waals and Platteeuw (1959) treatment of the statistical mechanics of clathrate formation, laboratory studies by Barrer and Edge (1967) on inert gas clathrates including dissociation pressures and heats of formation data, and work by Marshall, Saito, and Kobayashi (1964) on clathrate stability at pressures up to 4 kilobars.

Clathrates can be "pure" (only one kind of guest molecule) or "mixed" (more than one kind of guest molecule). In the pure case, a clathrate can be characterized by two parameters: the dissociation pressure, defined empirically as the minimum gas pressure of the guest molecule at a given temperature for which the corresponding clathrate is stable, and the degree of occupancy, defined as the fraction of available cage sites occupied by a guest molecule. In the mixed case, a single dissociation pressure still exists, but there are as many parameters characterizing relative occupancy as there are guest molecules. The chemical formula for the structure I clathrate in the usual notation is $\left(\underset{i}{\pi} \mathrm{X}_{i\left(y_{i}\right)}\right) \times 5 \frac{3}{4} \mathrm{H}_{2} \mathrm{O}$, where $y_{i}$ is the 


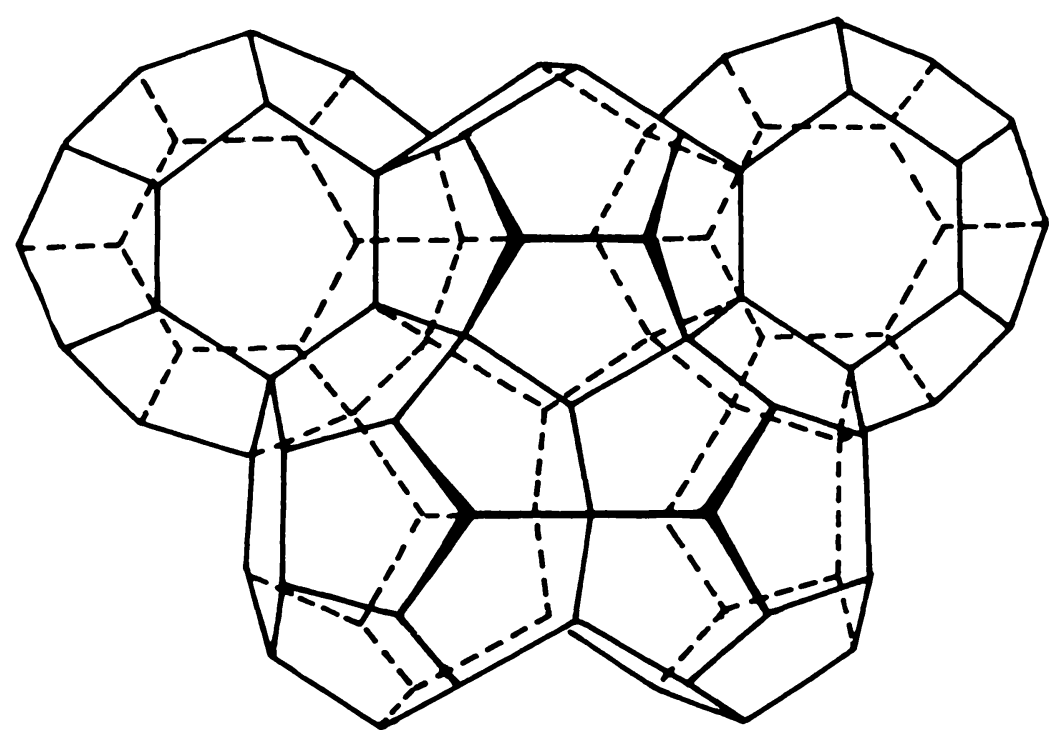

FIG. 1. - Structure I clathrate hydrate lattice (after Miller 1973). Oxygen atoms are located at vertices; hydrogen atoms are considered to lie on line between two vertices. The small cage (upper center) is a pentagonal dodecahedron with 20 water molecules; 24 water molecules make up the large-cage tetrakaidecahedron.

number fraction of all guests that are species $\mathrm{X}_{i}$. For structure II the $\mathrm{H}_{2} \mathrm{O}$ prefactor is $5 \frac{2}{3}$. As implied above, not all cages need be filled, and one of the guest species can be thought of as a hole, which enters the partition functions appropriately but otherwise does not contribute to the energy. Since the early 1960s much work has centered on using the van der Waals and Platteeuw model to predict these parameters (McKoy and Sinanoglu 1963; Saito, Marshall, and Kobayashi 1964; Nagata and Kobayashi 1966; Parrish and Prausnitz 1972; Holder, Corbin, and Papadopoulos 1980). Some of that work is now outdated since $\mathrm{Ar}$ and $\mathrm{Kr}$ were erroneously assumed to form structure I clathrate (Davidson et al. 1984). This type of modeling is employed in § III to calculate dissociation pressures and occupancies for guest molecules of cosmochemical interest; details of the calculation will be deferred to that section.

Some work has also been done in understanding explicitly the interaction between guest molecule and the surrounding host cage, theoretically by Davidson (1971) and experimentally, using infrared spectroscopy and dielectric measurements, by Bertie and Jacobs $(1982,1978,1977)$ and Davidson and Wilson (1963). Other studies involving computer simulation of guest and cage molecule motions have been undertaken by Tester, Bivins, and Herrick (1972), Plummer and Chen (1983), and Tse, Klein, and McDonald (1983). These studies are important in determining the degree of rotational inhibition of the encaged guest molecule, the magnitude of electric fields within the cage, and degree of interaction between guest molecules in adjacent cages (see § III).

Much less attention has been given to the kinetics of clathrate formation, most likely because of the long duration of experiments required to measure diffusion of gas molecules within grains and along grain boundaries in water ice. Both Barrer and Edge (1967) and Barrer and Ruzicka (1962) achieved nearly complete clathration in a system of water ice and noble gases by agitating the system, apparently exposing fresh ice to the gas. In the absence of shaking, an initially rapid uptake of gas by the ice to form clathrate was followed by a very slow uptake, suggesting a diffusional process. The problem of kinetics of clathrate formation will be explored in detail in § VI.

A number of studies have been done on physical properties of clathrate which are relevant to identifying clathrate in terrestrial ocean sediments as well as processes in solar system bodies. The thermal conductivity at low-to-moderate pressures was measured by Cook and Leaist (1983) and Stoll and Bryan (1979); the latter also measured acoustic wave velocity. Dharma-Wardana (1983) attempted to explain the low thermal conductivity of clathrate (one-fifth that of ice $I_{H}$ ) in terms of the large number of molecules per unit clathrate cell. Thermal conductivity and heat capacity studies were undertaken by Ross and Andersson (1982) to explore high-pressure solid compounds of water and tetrahydrofuran (THF), including THF clathrate. Results suggest the possibility of a high-density clathrate hydrate phase existing above 11 kilobars; implications for satellites as well as our theoretical high-pressure stability calculations will be presented below. A unique study by Pinder (1964) focused on the time-dependent rheology of a clathrate hydrate slurry, with THF and hydrogen sulfide as guest molecules. Some data on the density of clathrate compounds are available in Byk and Fomina (1968) and Kvenvolden and McDonald (1982).

We turn now to literature describing the predicted or observed occurrence of clathrates in natural environments. Reviews of the terrestrial occurrence of clathrate have been given by Claypool and Kvenvolden (1983) and Kvenvolden and McMenamin (1980). A recent review of properties of natural clathrate deposits is that of Pearson et al. (1983). Although evidence exists for clathrate in permafrost in western Siberia, Canada, and Alaska, and the existence of an $\mathrm{N}_{2}-\mathrm{O}_{2}$ clathrate in Antarctic ice has been proposed (Miller 1969) and tentatively detected (Shoji and Langway 1982), the best evidence 
for the existence of naturally formed clathrate comes from bottom-simulating reflectors in a number of ocean sediment areas around the world. The reflectors are apparently due to an abrupt decrease in sound velocity caused by trapped methane gas. The association of the gas with methane clathrate is supported by $(a)$ calculations showing that the reflectors are in a temperature-pressure regime stable for methane clathrate (Shipley et al. 1979) and (b) the retrieval in drill cores of frozen sediments and water ice evolving primarily methane gas (Shipley and Didyk 1982; Kvenvolden and McDonald 1982). The methane is likely of biogenic origin; the existence of the clathrate demonstrates that, in the presence of liquid water at least, clathrate compounds form spontaneously under the appropriate gas pressure and temperature conditions.

Application of clathrate hydrate properties to solar system objects extends over a 30 year period in the literature. The first direct application to solar system studies was probably that of Delsemme and Swings (1952), who proposed the existence of clathrate hydrate in cometary nuclei. This was followed by more detailed work by Delsemme and Wenger (1970) and Delsemme and Miller (1970). The former produced methane clathrate at temperatures as low as $82 \mathrm{~K}$ by condensing water vapor onto a cold plate in the presence of methane gas. The stripping of ice grains during dissociation was suggested as a mechanism for cometary halo production. The latter paper modeled clathrate formation as a special case of gas adsorption and suggested that radicals observed spectroscopically in comets could be emitted from clathrate cages. Although more detailed observations of comets are now available, the existence of clathrate in comets is still hypothetical.

In a pioneering paper, Stanley Miller (1961) utilized the van der Waals and Platteeuw model and his own experimental data to determine the likelihood of occurrence of clathrate hydrate in a wide range of solar system objects, including the envelopes of the outer planets, Saturn's rings, satellite interiors, terrestrial planet atmospheres, Mars's polar caps, comets, as well as interstellar grains. An updated discussion was later presented by Miller (1973). A somewhat more detailed analysis of the stability of carbon dioxide clathrate in the Martian ice caps was given by Miller and Smythe (1970). Somewhat later, Smythe (1975) produced a set of laboratory reflectance spectra of methane and carbon dioxide clathrate frost to determine the detectability of clathrate on surfaces of the outer planet satellites.

The application of clathrate formation in primordial gaseous disks to the present atmospheres of planets and satellites has received sporadic attention over the past decade. A prescient paper by Lewis (1971) suggested incorporation of methane clathrate in Titan and predicted the resulting argonto-methane ratio in Titan's atmosphere derived from the clathrate composition. Hunten (1978) also suggested an atmosphere in equilibrium with a surface of methane clathrate, which at the time was not ruled out by the data. Sill and Wilkening (1978) used existing laboratory data on clathrate dissociation pressures for a range of guest molecules of cosmochemical interest to calculate the gas composition in clathrate hydrate derived from a solar composition gas. They concluded that addition of one part per million clathrate gases (in comets) to Earth's atmosphere could explain the observed terrestrial noble gas ratios. The above studies did not, however, incorporate the recent experimental result that pure argon and krypton clathrates possess structure II (Davidson et al. 1984).

More recently, spacecraft missions to the outer solar system have renewed interest in understanding the origin and effect of volatiles in icy satellites. Stevenson $(1982 a)$ proposed clathrate hydrate dissociation as a driver for explosive ammoniawater volcanism on Rhea-sized satellites in the Saturn system. Much recent work on clathrate applications has centered on Titan. Owen (1982) renewed the suggestion that Titan's atmosphere is derived from clathrate and suggested that the $\mathrm{N}_{2}$ making up most of the present atmosphere was accreted in clathrate. Lunine and Stevenson (1982a) calculated gas composition in clathrate derived from a circum-Saturnian nebula and concluded that although $\mathrm{CH}_{4}$ in Titan is likely derived from primordial clathrate, $\mathrm{N}_{2}$ would not be substantially incorporated and may have been photochemically produced from $\mathrm{NH}_{3}$ later (Atreya, Donahue, and Kuhn 1978).

It is important to note that no direct evidence exists for clathrate anywhere aside from Earth. In part this may be because clathrate "hides" itself very well as ordinary water ice I in reflection spectra (Smythe 1975). The utility of studying clathrate thermodynamics rests more on indirect evidence that clathrates play a role in determining the composition and evolution of surfaces and atmospheres of satellites (and perhaps giant-planet envelopes) in the outer solar system. Striking improvements in spectral resolution in ground-based reflection spectroscopy now raise the possibility of distinguishing between water ice I (and its high-pressure polymorphs-Gaffney and Matson 1980) and clathrate hydrate on surfaces in the outer solar system in the near future.

\section{STATISTICAL MECHANICAL MODEL FOR CLATHRATE FORMATION}

In this section we describe the thermodynamic model used to predict the stability regimes of clathrate hydrate and the composition of the guest molecule component.

\section{a) Thermodynamic and Structural Properties}

Clathrate hydrate is a distinct phase in a multicomponent system consisting of water and any number of nonpolar, weakly polar, and, more rarely, strongly polar chemical species. It is nonstoichiometric since, although the number of cages is precisely determined, their fractional occupancy is a function of temperature, pressure, and relative abundance of the species. Although the clathrate structure is distinguished from that of other ice phases by containing large open cage structures, the water molecule lattice bonding mechanism is the usual hydrogen bonding (Jeffrey and McMullan 1967). The guest molecule-cage interaction is primarily the sum of core overlap effects (a strongly repulsive term) and an attractive van der Waals-like (induced dipole-induced dipole) interaction. The included guest molecule must stabilize the cage structure because of the attractive term, since the empty cage structure is energetically unfavorable relative to the usual (more compact) forms of water ice. 
The phase boundary in temperature pressure space of clathrate hydrate is defined by the equality of chemical potentials (or Gibbs energies $g$, where $d g=-S d T+V d P$ ) of each component (guest and water host) in the coexisting phases. The coexisting phases are $(a)$ clathrate hydrate with incorporated guest molecule, and $(b)$ water ice or liquid (with some dissolved molecules of the guest species) plus the pure phase of the guest species stable under ambient conditions $T, P$. We consider only a single guest species in the present discussion. Figure 2 plots qualitatively the phase boundary as a function of $P$ and $T$. Consider, first, the low-pressure regime in which the system pressure is essentially the vapor pressure of the guest molecule (since the vapor pressure of water is invariably much lower). With increasing gas pressure at a given temperature, the Gibbs energy of the gas is increased by decreasing its entropy. The clathrate Gibbs energy is negligibly affected by pressure, since the pressure is much less than the bulk modulus, so it follows that an increase in pressure favors clathrate formation. In this ideal gas regime, the net volume change and entropy change accompanying clathrate formation are clearly both negative, and the Clausius Clapeyron equation predicts a positive value of $d T / d P$. The phase boundary pressure, at a given temperature, for clathrate formation has been referred to in the literature as the dissociation pressure of the clathrate. We use this term only in the low-pressure

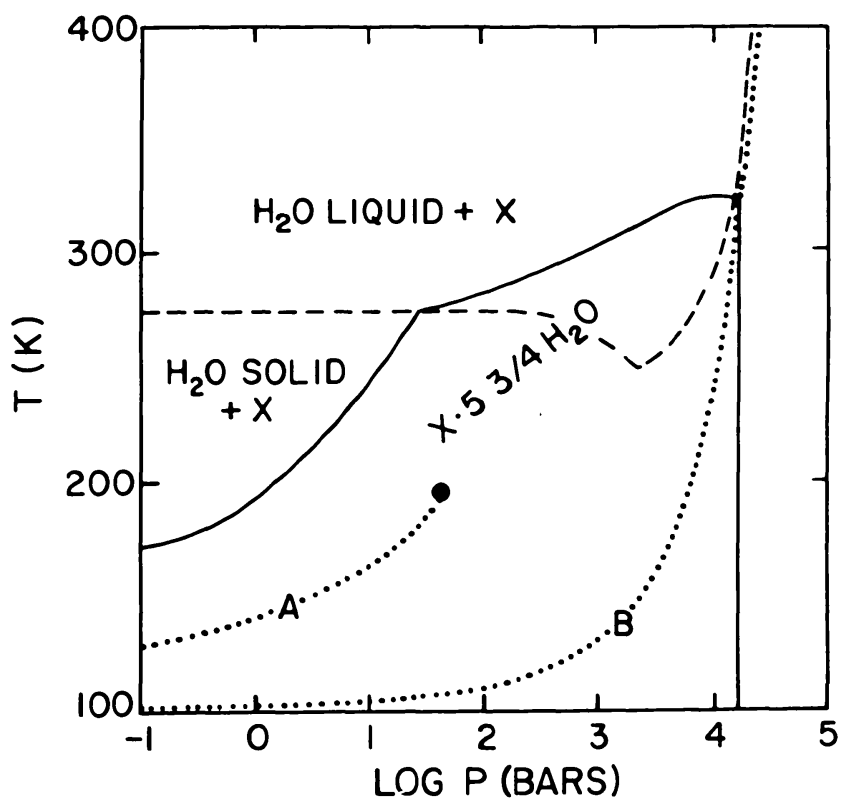

FIG. 2.-Schematic diagram of stability fields of clathrate hydrate, occupied by guest molecule $\mathrm{x}$, plotted as $\log P$ (in bars) vs. temperature in degrees kelvin. Solid line delineates region in which clathrate is thermodynamically preferred relative to water ice or liquid plus coexisting component $x$. Dashed line is water ice-liquid phase boundary; within the clathrate stable region the existence of water phase separate from clathrate is contingent on the relative amounts of water and $x$. Superposed are dotted lines representing the vapor-liquid (A) and liquid-solid (B) phase boundaries of pure component $\mathrm{x}$. The axis values are for $\mathrm{x}=$ methane; the diagram is qualitatively similar for $\mathrm{N}_{2}, \mathrm{CO}$, and other volatile, mainly nonpolar species with small molecular diameters. Note change in slope of clathrate stability field in crossing water ice-liquid boundary. regime. As pressure is increased and the guest is no longer in the ideal gas regime, compressive effects primarily on the guest molecule (and secondarily on the water) become important, and the volume change from phase $\mathrm{A}$ (guest $+\mathrm{H}_{2} \mathrm{O}$ ) to $\mathrm{B}$ (clathrate) decreases. At a given temperature, then, a higher system pressure is required to stabilize the clathrate than would be expected in the ideal gas regime, and $d T / d P$ decreases as seen in Figure 2. Eventually the volume change $\mathrm{A} \rightarrow \mathrm{B}$ becomes zero and $d T / d P=0$, defining a maximum temperature at which the clathrate is stable. This "critical point" $T_{c}$ is a function of the thermodynamic properties of the particular guest and, hence, is different for each species. At each temperature below $T_{c}$, there exist both maximum and minimum pressures defining the domain of clathrate stability. The minimum pressure exists because at lower pressures the gas entropy is very large (i.e., more favorable), and the maximum pressure exists because the coexisting pure guest phase has reached a low enough specific volume that the volume change (clathrate $\rightarrow$ guest $+\mathrm{H}_{2} \mathrm{O}$ ) is energetically favorable. The precise sign and value of $d T / d P$ at the high-pressure end of the clathrate stability field are sensitive functions of the compressibility of the clathrate, water, and guest molecule components, and are calculated for $\mathrm{CH}_{4}$ and $\mathrm{N}_{2}$ in $\S \mathrm{V} . P-T$ data in the literature are restricted to the low-pressure end of Figure 2 ( $\lesssim 4$ kilobars); studies by Tammann and Krige (1925) and van Berkum and Diepen (1979) appear to have located the critical point for $\mathrm{SO}_{2}$ clathrate.

Considering in more detail the nature of the guest-host interaction, it is inaccurate to think of the guest molecules as impurities occupying imperfections or substituting for water molecules in the lattice structure. X-ray diffraction studies of clathrates (see Jeffrey and McMullan 1967 for a review of the substantial literature on this subject) demonstrate a welldefined structure in which cages of diameter $\sim 10 \AA$ are present. Calculations by Davidson (1971) indicate substantial cancellation of lattice water molecule dipolar fields within the cage volume. Theoretical and experimental studies of infrared spectra (e.g., Davidson 1971; Bertie and Jacobs 1978) and physical properties of clathrate such as sound velocity (Pearson et al. 1983) suggest that these properties are to first order determined by the water molecules and their lattice structure, crudely independent of particular guest species, and the interaction between the guest molecule and host lattice is extremely weak. Even the very low thermal conductivity of the clathrate relative to water ice may be a result of the form of the lattice structure and not due to coupling to the guest molecule (Dharma-Wardana 1983), although this is controversial. The weak interaction is consistent with London dispersion forces (Fowler and Guggenheim 1960, p. 278) being responsible for the attractive part of the guest-host interaction, with lesser contribution from dipole-induced-dipole and dipole-dipole interactions with residual fields in the cage. This has long been recognized (van der Waals and Platteeuw 1959), since the noble gases are observed as encaged guest molecules; we wish to emphasize the point here since the predominantly icelike properties are potentially important for evolution models of icy satellites. Strongly dipolar molecules apparently can occupy cage sites (Jeffrey and McMullan 1967); the possible incorporation of ammonia in clathrate cages is deferred to a later section. 


\section{b) Model of Clathrate Formation}

The statistical mechanical model for clathrate formation developed by van der Waals and Platteeuw (1959) assumes that the guest molecule incorporation into clathrate cages is physically similar to ideal adsorption onto fixed sites, generalized to three dimensions. Their explicit assumptions are adopted here and are as follows:

1. The free energy of the $\mathrm{H}_{2} \mathrm{O}$ lattice structure is independent of the occupation (and mode of occupation) of the guest molecule.

2. (a) The guest molecules are confined to the cage volume with one guest molecule per cage. (b) Guest molecules rotate freely within the cage.

3. Guest molecules do not interact with each other, so that the partition function describing the guest molecule motion is independent of the presence and type of other guest molecules.

4. Classical statistics applies.

Assumption 1 says that the cage structure is relatively undistorted by the presence of the guest molecule, which as noted above seems to be valid based on spectroscopic and other studies for small guest molecules such as $\mathrm{CH}_{4}$. It is undoubtably in error for large molecules such as $\mathrm{CO}_{2}$; however, we will see below that our qualitative conclusions regarding the incorporation of this molecule in ice are probably not sensitive to this assumption. A very interesting possible violation of assumption $2 a$, with astrophysical implications, is the occupancy of single cages by more than one molecule. Our calculations indicate a finite number of cages occupied by both $\mathrm{CH}_{4}$ and $\mathrm{H}_{2}$ when both gases are present. In this circumstance, the "dimer" $\left(\mathrm{CH}_{4}, \mathrm{H}_{2}\right)$ must be thought of as a separate species from $\mathrm{CH}_{4}$ and $\mathrm{H}_{2}$ in the statistical mechanical accounting. Assumption $2 b$ is valid for small spherical molecules but could be in error at low temperatures for rodlike molecules, even small ones such as $\mathrm{N}_{2}$ and $\mathrm{CO}$. We address this problem further in the context of the calculations. Assumption 3 is verified for moderate-sized guest molecules in Davidson (1971). Assumption 4 is not valid for $\mathrm{H}_{2}, \mathrm{He}$, and $\mathrm{Ne}$, but for larger molecules on which our quantitative analysis is concentrated, classical statistics is generally valid.

Van der Waals (1956) and van der Waals and Platteeuw (1959) outline the construction of the configuration partition function for clathrate hydrate in which a fraction $y$ of the cages are occupied by a guest molecule. The condition is then imposed that the chemical potentials of both guest and host molecules in the coexisting phases be equal. The result is the set of equations

$$
\begin{aligned}
\frac{\mu_{\mathrm{H}_{2} \mathrm{O}}^{i}-\mu_{\mathrm{H}_{2} \mathrm{O}}^{\beta}}{k T} & \equiv-\frac{\Delta \mu^{\beta}}{k T} \\
& =\nu_{1} \ln \left(1-\sum_{j} y_{1 j}\right)+\nu_{2} \ln \left(1-\sum_{j} y_{2 j}\right), \\
y_{i j}(T, P) & =\frac{C_{i j}(T, P) f_{j}(T, P)}{1+\sum_{k} C_{i k}(T, P) f_{k}(T, P)} \\
C_{i j}(T, P) & =\frac{z_{i j}}{k_{\mathrm{B}} T} \int_{0}^{a_{i} / 2} \exp \left[-\frac{\omega_{i j}(r)}{k_{\mathrm{B}} T}\right] 4 \pi r^{2} d r
\end{aligned}
$$

where $\mu_{\mathrm{H}_{2} \mathrm{O}}^{\beta}=$ chemical potential of unoccupied water-ice clathrate structure, $\mu_{\mathrm{H}_{2} \mathrm{O}}^{i}=$ chemical potential of water in coexisting phase, $y_{i j}=$ fractional occupancy of cage sites $i=$ 1,2 by molecule of species $j, f_{j}=$ fugacity of guest molecule $j$ in its pure phase (i.e., $\mu_{j}=k T \ln f_{j}$ ), $a_{i}=$ cage radius, $\omega_{i j}(r)$ $=$ spherically averaged potential energy of the guest molecule located a distance $r$ from the cage center, $k_{\mathrm{B}}=$ Boltzmann's constant, and $k=$ gas constant. The factors $\nu_{1}$ and $\nu_{2}$ are the number of cage sites per water molecule for the large and small cages, respectively. For structure I, $\nu_{1}=1 / 23$ and $\nu_{2}=$ $3 / 23$; for structure II, $\nu_{1}=2 / 17$ and $\nu_{2}=1 / 17$ (see, for example, Davidson 1971). The numerical factor $z$ in equation (3) is the ratio of the rotational and intramolecular vibrational partition function (volume factor removed) of the guest molecule in the cage to that of the free molecule at the same temperature. If the rotational and intramolecular vibrational degrees of freedom of the encaged molecule are the same as for the free molecule, $z_{i j}=1$. In what follows we assume, based on the above discussion, that $z_{i j}=1$; exceptions are discussed as they occur.

In general we may compute the fugacity for a single guest species $j$ as

$$
f_{j}(T, P)=\phi_{j}\left(T, P_{0}\right) P_{0} \exp \frac{1}{k T} \int_{P_{0}}^{P} V\left(T, P^{\prime}\right) d P^{\prime},
$$

where $V$ is the volume of the coexisting guest species phase at system temperature $T$ and pressure $P, P_{0}$ is a reference pressure, and $\phi\left(T, P_{0}\right)$ is the fugacity coefficient (Prausnitz 1969, p. 5) at reference conditions.

The quantity $\Delta \mu^{\beta}$ is computed using an expression from Holder, Corbin, and Papadopoulos (1980):

$$
\begin{aligned}
\frac{\Delta \mu^{\beta}(T, P)}{k T}= & \frac{\Delta \mu^{\beta}\left(T_{0}, P_{0}\right)}{k T_{0}}-\int_{T_{0}}^{T} \frac{\Delta h^{\beta}\left(T^{\prime}, P_{0}\right)}{k T^{\prime 2}} d T^{\prime} \\
& +\frac{1}{k T} \int_{P_{0}}^{P} \Delta V^{\beta}\left(T, P^{\prime}\right) d P^{\prime}
\end{aligned}
$$

where $\Delta h^{\beta}, \Delta V^{\beta}$ are the enthalpy and volume difference between the empty hydrate and coexisting water phase, and $T_{0}$ is a reference temperature. The pressure dependence of $C_{i j}$ is discussed in $\S \mathrm{V}$. Equations (1)-(5) are the basic equations we will use to determine clathrate stability throughout the paper. In the low-pressure ( $\ll 1$ kilobar) regime considered in $\S \S$ III and IV, three simplifications are made: $C_{i j}(T, P)=C_{i j}(T)$, the last term in equation (5) is neglected, and $f_{j}=P_{j}=$ pressure exerted by the guest species vapor (or supercritical fluid) phase.

The $C_{i j}$ are called Langmuir constants and depend on the molecular properties through $\omega(r)$, the spherically averaged interaction between guest and cage. This effective potential depends on the shape as well as size of the molecule. Equation (3) explicitly assumes a spherical cage; this is adequate for all cages. The largest deviation from sphericity is in structure I, where the vertices of the large cage as defined by water oxygen atoms vary in distance to center by $\$ 14 \%$ (Davidson 1971).

McKoy and Sinanoglu (1963) and Parrish and Prausnitz (1972) derived expressions for the spherically averaged potentials for spherical and rodlike guest molecules using the Kihara 
potential appropriate for dispersion interactions between guest and host:

$$
\Gamma(r)=\varepsilon\left[\left(\frac{\rho_{m}}{\rho}\right)^{12}-2\left(\frac{\rho_{m}}{\rho}\right)^{6}\right], \quad \rho>0,
$$

for a single guest molecule interaction, where $\rho=$ shortest distance between molecular cores, $\varepsilon=$ potential minimum, and $\rho_{m}$ is separation at which $\Gamma=-\varepsilon$ is attained. Note that when the core radius is zero (pointlike molecules), we recover the Lennard-Jones 12-6 potential (used in van der Waals and Platteeuw 1959), and that $\rho /(2)^{1 / 6}=\sigma$, the distance of closest approach of two molecules which collide with zero initial relative kinetic energy (Hirschfelder, Curtiss, and Bird 1954, p. 23).

The derivation of the spherically averaged potential is formally the same as in Fowler and Guggenheim (1960, pp. 338-339) for models of liquids using the Lennard-Jones (L-J) potential, and consists of keeping the guest molecule fixed at distance $r$ from the center while the host water molecule is moved about a spherical surface of radius $a$ centered on the origin. We will not reproduce the derivation here; rather we give the results from McKoy and Sinanoglu (1963):

$$
\begin{aligned}
\omega(r) & =\frac{Z \varepsilon}{2}\left[\frac{\rho_{m}^{12}}{a^{11} r}\left(\delta^{10}+\frac{c}{a} \delta^{11}\right)-\frac{2 \rho_{m}^{6}}{a^{5} r}\left(\delta^{4}+\frac{c}{a} \delta^{5}\right)\right], \\
\delta^{N} & =\frac{1}{N}\left[\left(1-\frac{r}{a}-\frac{c}{a}\right)^{-N}-\left(1+\frac{r}{a}-\frac{c}{a}\right)^{-N}\right],
\end{aligned}
$$

for spherical guest molecules, and

$$
\begin{gathered}
\omega(r)=\frac{Z \varepsilon}{2} \sum_{n=0}^{\infty}\left(-\frac{d}{a}\right)^{n}\left[f_{n} \frac{\mu_{n}(y)}{\alpha^{4}}-\frac{g_{n} \lambda_{n}(y)}{\alpha^{2}}\right], \\
\mu_{n}(y)=\frac{1}{\sqrt{y}}\left[\frac{1}{(1-\sqrt{y})^{n+10}}-\frac{1}{(1+\sqrt{y})^{n+10}}\right] \mathscr{F}(d), \\
\lambda_{n}(y)=\frac{2}{\sqrt{y}}\left[\frac{1}{(1-\sqrt{y})^{n+4}}-\frac{1}{(1+\sqrt{y})^{n+4}}\right] \mathscr{G}(d),
\end{gathered}
$$

for rodlike molecules, here $d=\operatorname{rod}$ length, $Z=$ number of nearest-neighbor water molecules in a cage of radius $a$ ( 20 for small, 24 for large cage), $c=$ core radius of guest molecule, $y=(r / a)^{2}, \alpha=\left(a / \rho_{m}\right)^{3}$, and we find the constants $f_{n}$ and $g_{n}$ to be

$$
\begin{aligned}
& f_{n}=\left(\begin{array}{c}
-6 \\
n
\end{array}\right) \frac{1}{n+1} \frac{1}{10+n}, \\
& g_{n}=\left(\begin{array}{c}
-3 \\
n
\end{array}\right) \frac{1}{n+1} \frac{1}{4+n},
\end{aligned}
$$

where

$$
\left(\begin{array}{c}
-l \\
n
\end{array}\right)=\frac{l \times(l+1) \times \cdots \times(l+n-1)}{n !} \times(-1)^{n}
$$

(Marion 1970, pp. 542-543). The quantities $\mathscr{F}(d)$ and $\mathscr{G}(d)$ are power series in $(d / a)^{2}$ included to allow accurate comparison between structure I and II Langmuir constants.

For a given choice of parameters $\varepsilon, \rho_{m}, c$, and temperature $T$ we compute the integral in equation (3) using equation (7) or (8), terminating the integration at a value $r$ such that the probability of finding the molecule, $\exp [-\omega(r) / k T]$, at $r$ is $\ll 1$. This gives $C_{i j}(T)$, and in the low-pressure calculations for a single species $j$, equations (1) and (2) are then solved (using laboratory values for $\Delta \mu^{\beta}$; see below) for the dissociation pressure for species $j$. To solve for several guest species, abundance relations between them must be applied to close the system. Application of equations (1)-(5) to the high-pressure regime is discussed in $\S \mathrm{V}$.

\section{c) Choice of Molecular Parameters}

We now fit the above equations to existing laboratory data on clathrate to derive values for $\varepsilon, \rho$, and, where necessary, $c$. We will then use these parameters to predict the dissociation of clathrate at temperatures below that available in the data, or the dissociation pressure for guest molecules similar to others but for which laboratory data are scant or nonexistent. We use our results in $\S$ VII to predict the composition of the clathrate in plausible nebular models. Our derived parameters also will be used to predict the stability of clathrate under high pressure and in coexistence with an ammonia-water solution.

In evaluating equation (1) a choice of $\Delta \mu^{\beta}$, the difference in chemical potential between the empty clathrate lattice and coexisting liquid water or ice phase, must be made. To date no attempt has been made to calculate this from first principles; instead, it has been derived by several laboratory studies. For structure I van der Waals and Plateeuw (1959) suggest $167 \mathrm{cal}$ mole $^{-1}$ at $273 \mathrm{~K}$, based on the bromine clathrate. Parrish and Prausnitz (1972) and Holder, Corbin, and Papadopoulos (1980) derive $\Delta \mu^{\beta} \sim 310 \mathrm{cal} \mathrm{mole}^{-1}$ based on cyclopropane and methane clathrate. Since the existence of a structure I bromine clathrate has been called into question (Parrish and Prausnitz 1972), we use the latter value. For structure II, Sortland and Robinson (1964) derive $211 \mathrm{cal} \mathrm{mole}^{-1}$.

Of equal concern is the temperature dependence of $\Delta \mu^{\beta}$ below the ice point, on which authors disagree. Parrish and Prausnitz conclude $\Delta \mu^{\beta}$ decreases by $\sim 6 \%$ for both structures from 273 to $70 \mathrm{~K}$; Holder, Corbin, and Papadopoulos (1980) predict an increase of $27 \%$ for structure I over that temperature range. Using the Debye model of solids, infrared spectral data on intermolecular translational frequencies in ice I and structure II clathrate (Johari and Chew 1984), and thermodynamic data in Giauque and Stout (1936), we estimate that $\Delta \mu^{\beta}$ increases $\sim 15 \%$ from 273 to $70 \mathrm{~K}$. Unfortunately, data on librational vibrations in the clathrate lattice do not exist. We adopt a constant $\Delta \mu^{\beta}$ in the low-pressure ice I regime. In the liquid-water field the temperature dependence of $\Delta \mu^{\beta}$ is large and dominated by the ice-to-liquid enthalpy change; this will be important for calculations in $\S \S \mathrm{IV}$ and V.

Table 1 lists the guest molecules included in the present study, along with sources of laboratory data and their temperature range. Molecules listed under "substantial data" were studied over a temperature range sufficient to allow fitting of Kihara or Lennard-Jones parameters such that the calculated dissociation pressures should be good to within $10 \%$ at mod- 
erate temperatures $(~ \gtrsim 150 \mathrm{~K}$ ) and probably within a factor of several extrapolated to low $(T<100 \mathrm{~K})$ temperatures. Molecules in the "some data" category have been studied under restricted temperature ranges, and the consequent degree of uncertainty in dissociation pressure at low temperatures increases by about a factor of 10 . Those molecules listed under the "little or no data" category required special treatment, which is outlined later.

For some molecules in the table, data on the clathrate hydrate structure is not available. We have predicted which structure is stable based on laboratory dissociation pressures

TABLE 1

Summary of Guest Molecules ANalyzed

\begin{tabular}{|c|c|c|}
\hline Species & Reference & Temperature Range (K) \\
\hline \multicolumn{3}{|c|}{ Substantial Data } \\
\hline $\operatorname{Ar} \ldots \ldots$ & $\begin{array}{l}1 \\
2 \\
3\end{array}$ & $\begin{array}{c}273 \\
148-90.2 \\
273\end{array}$ \\
\hline $\mathrm{Kr} \ldots \ldots$ & $\begin{array}{l}1 \\
2\end{array}$ & $\begin{array}{c}273 \\
202-149 \pm 1\end{array}$ \\
\hline $\mathrm{Xe} \ldots \ldots$ & $\begin{array}{l}1 \\
2\end{array}$ & $\begin{array}{c}273 \\
268.3-211.2\end{array}$ \\
\hline $\mathrm{CH}_{4} \ldots$ & $\begin{array}{l}1 \\
4 \\
5\end{array}$ & \begin{tabular}{l}
\multicolumn{1}{c}{273} \\
$207-173$ \\
$111-82$
\end{tabular} \\
\hline $\mathrm{CO}_{2} \ldots$ & $\begin{array}{l}1 \\
4\end{array}$ & $\begin{array}{c}273 \\
232-175\end{array}$ \\
\hline $\mathrm{C}_{2} \mathrm{H}_{4} \ldots$ & $\begin{array}{l}1 \\
4 \\
6\end{array}$ & $\begin{array}{c}273 \\
240-163 \\
272-269\end{array}$ \\
\hline \multicolumn{3}{|c|}{ Some Data } \\
\hline $\mathrm{N}_{2} \ldots \ldots$ & $\begin{array}{l}7 \\
8\end{array}$ & $\begin{array}{l}272 \\
238\end{array}$ \\
\hline $\mathrm{H}_{2} \mathrm{~S} \ldots$ & $\begin{array}{r}9 \\
10\end{array}$ & $\begin{array}{l}272-247 \\
273-250\end{array}$ \\
\hline $\mathrm{SO}_{2} \ldots$ & $\begin{array}{r}1 \\
11\end{array}$ & $\begin{array}{c}273 \\
271-265\end{array}$ \\
\hline $\mathrm{PH}_{3} \ldots$ & $\begin{array}{r}1 \\
12\end{array}$ & $\begin{array}{l}273,267 \\
298,274\end{array}$ \\
\hline \multicolumn{3}{|c|}{ Little or No Data } \\
\hline $\begin{array}{l}\mathrm{O}_{2} \ldots \ldots \\
\mathrm{CO} \ldots \ldots \\
\mathrm{H}_{2} \ldots \ldots \\
\mathrm{He} \ldots \ldots \\
\mathrm{Ne} \ldots \ldots\end{array}$ & $\begin{array}{c}8 \\
\ldots \\
13 \text { (mixed clathrate) } \\
\ldots \\
\ldots\end{array}$ & $\begin{array}{c}272 \\
\ldots \\
279 \\
\ldots \\
\ldots\end{array}$ \\
\hline
\end{tabular}

REFERENCES.-(1) von Stackelberg and Muller 1954. (2) Barrer and Edge 1967. (3) van der Waals and Platteeuw 1959. (4) Miller 1961. (5) Delsemme and Wenger 1970. (6) Diepen and Scheffer 1950. (7) Miller 1969. (8) van Cleef and Diepen 1965. (9) Korvezee and Scheffer 1931. (10) Selleck, Carmichael, and Sage 1952. (11) Tammann and Krige 1925. (12) Byk and Fomina 1968. (13) von Stackelberg and Meinhold 1954. and our model, by determining which structure has the lower chemical potential for a given set of molecular force constants which fit the dissociation pressure data. This procedure correctly indicated $\mathrm{Ar}$ and $\mathrm{Kr}$ to be structure II clathrate, and $\mathrm{CH}_{4}$ and $\mathrm{Xe}$ to be structure I above $100 \mathrm{~K}$ (Davidson et al. 1984 performed their X-ray and neutron diffraction studies at temperatures of less than $100 \mathrm{~K}$; however, their samples were prepared at $240 \mathrm{~K}$ and the resulting structure was likely quenched-in during cooling). Below $100 \mathrm{~K}$ neither structure is strongly preferred by $\mathrm{CH}_{4}$ and $\mathrm{Xe}$ based on our fits. The best fit to $\mathrm{H}_{2} \mathrm{~S}$ pressure data implies structure II is preferred contrary to Davidson et al. (1984). However, force constants can be chosen for $\mathrm{H}_{2} \mathrm{~S}$ that lead to structure $\mathrm{I}$ as the preferred form, with a slightly poorer fit to dissociation pressure data. In any case, $\mathrm{H}_{2} \mathrm{~S}$ will very readily incorporate in either cage structure as a secondary gas. We predict $\mathrm{H}_{2}, \mathrm{He}, \mathrm{Ne}, \mathrm{O}_{2}$, and $\mathrm{PH}_{3}$ to form structure II; $\mathrm{CO}_{2}, \mathrm{SO}_{2}, \mathrm{C}_{2} \mathrm{H}_{4}$, and (marginally) $\mathrm{CO}$ to form structure I; and $\mathrm{N}_{2}$ to have essentially no preference. We assume in what follows that both pure $\mathrm{CO}$ and $\mathrm{CH}_{4}$ clathrates are structure I, but we discuss any changes in our conclusions if either of these molecules were to prefer structure II at low temperatures. Note that two sets of Langmuir constants were calculated for each molecule, one for each structure, using the same mixed force parameters $c_{m}, \varepsilon_{m}, \rho_{m}$. The calculations of incorporation of molecules from a solar composition gas into clathrate is done in $\S$ VII for both structure I and II. Using the same force parameters in both structures assumes the guest molecule interaction with individual water molecules is not affected by cage size or number of water molecules per cage. This is a good approximation so long as the guest molecule is substantially smaller than the cage size, which is true for most molecules of interest here.

Table 2 lists dissociation pressures derived from the data fits over a range of temperatures, below the low-pressure ice point, of interest to solar system studies. Also shown are corresponding data points, when available, from the data sources given in Table 1 . The ability to fit the data with two or three parameters so well over large temperature ranges gives confidence in the physical significance of the form of the equation, our choice of $\Delta \mu^{\beta}=$ constant, and our ability to use those fits to extrapolate to low temperature and high pressure. These results may be conveniently utilized by fitting to the approximate form $\log P=A / T+B$, where $A, B$ are constants (Miller 1961).

Data on $\mathrm{Ar}, \mathrm{Kr}, \mathrm{Xe}$, and $\mathrm{CH}_{4}$ clathrate extend to low temperatures, as low as $82 \mathrm{~K}$ for the $\mathrm{CH}_{4}$ clathrate. Below 110 $\mathrm{K}$, condensation of water vapor may produce a metastable amorphous ice phase. Using the maximum estimated enthalpy difference between amorphous ice and ice I of $300 \mathrm{cal} \mathrm{mole}^{-1}$ (Eisenberg and Kauzmann 1969, p. 90), we calculate the ratio of $\Delta \mu^{\beta}$ for amorphous ice divided by that for hexagonal ice to be 0.91 at $100 \mathrm{~K}$ and 0.65 at $70 \mathrm{~K}$. The dissociation pressure data for methane in Table 1 strongly suggest no change in $\Delta \mu^{\beta}$ for $T$ as low as $70 \mathrm{~K}$, consistent with laboratory conditions in which ice I itself is cooled below $110 \mathrm{~K}$ or the condensing water vapor forms the clathrate phase directly in the presence of methane gas. The existence of amorphous ice under nebular condensation conditions is controversial; in what follows we assume hexagonal water ice but discuss the effect of having amorphous ice where relevant. 
TABLE 2

Results of Fits to Dissociation PRESSURES

\begin{tabular}{|c|c|c|c|}
\hline Species & $T$ & $P$ (bars) & $P_{\text {experimental }}$ (bars) \\
\hline $\mathrm{CH}_{4} \ldots$ & $\begin{array}{r}273 \\
230 \\
180 \\
100 \\
82\end{array}$ & $\begin{array}{l}24.3 \\
5.63 \\
0.419 \\
2.79(-5)\{1.26(-5)\} \\
2.39(-7)\{1.60(-8)\}\end{array}$ & $\begin{array}{l}25.2-26.0 \\
5.65 \\
0.405 \\
2.10(-5) \\
2.10(-7)\end{array}$ \\
\hline $\mathrm{N}_{2} \ldots \ldots$ & $\begin{array}{r}272 \\
238 \\
180 \\
100 \\
60\end{array}$ & $\begin{array}{l}145 \\
62.7 \\
6.80 \\
3.86(-3) \\
5.12(-8)\end{array}$ & $\begin{array}{r}141.5 \\
61.9 \pm 0.5 \\
\ldots \\
\ldots \\
\ldots\end{array}$ \\
\hline $\mathrm{CO}_{2} \ldots$ & $\begin{array}{r}273 \\
200 \\
150 \\
100 \\
80\end{array}$ & $\begin{array}{l}12.7 \\
0.366 \\
4.34(-3) \\
6.04(-7) \\
7.66(-10)\end{array}$ & $\begin{array}{l}12.5 \\
0.351 \\
4.78(-3) \\
\quad \ldots \\
\quad \ldots\end{array}$ \\
\hline Ar...... & $\begin{array}{r}273 \\
230 \\
149 \\
116 \\
90 \\
60\end{array}$ & $\begin{array}{l}91.1 \\
33.5 \\
1.00 \\
5.84(-2) \\
1.44(-3) \\
3.83(-7)\end{array}$ & $\begin{array}{l}92.5-95.5 \\
33.7 \\
0.97-1.0 \\
5.2-5.5(-2) \\
1.38-1.45(-3) \\
\ldots\end{array}$ \\
\hline $\mathrm{Kr} \ldots \ldots$ & $\begin{array}{l}273 \\
203 \\
181 \\
149 \pm 1 \\
100 \\
60\end{array}$ & $\begin{array}{l}15.0 \\
1.23 \\
0.373 \\
3.51(-2) \\
5.03(-5) \\
9.02(-11)\end{array}$ & $\begin{array}{l}14.5-14.7 \\
1.11 \\
0.342 \\
6.30(-2) \pm 0.3(-2) \\
\quad \cdots \\
\quad \cdots\end{array}$ \\
\hline $\mathrm{Xe} \ldots \ldots$ & $\begin{array}{r}273 \\
238 \\
211 \\
180 \\
100 \\
60\end{array}$ & $\begin{array}{l}1.48 \\
0.316 \\
6.73(-2) \\
6.37(-3) \\
1.62(-8) \\
6.34(-17)\end{array}$ & $\begin{array}{l}1.15-1.5 \\
0.307 \\
6.52(-2) \\
\ldots \\
\cdots \\
\ldots\end{array}$ \\
\hline $\mathrm{H}_{2} \mathrm{~S} \ldots$ & $\begin{array}{r}273 \\
247 \\
180 \\
100 \\
80\end{array}$ & $\begin{array}{l}0.968 \\
0.296 \\
2.79(-3) \\
2.72(-9) \\
1.12(-12)\end{array}$ & $\begin{aligned} 0.919 & \\
0.298 & \\
& \ldots \\
& \ldots \\
& \ldots\end{aligned}$ \\
\hline $\mathrm{C}_{2} \mathrm{H}_{4} \ldots$ & $\begin{array}{r}272 \\
269 \\
180 \\
100 \\
80\end{array}$ & $\begin{array}{l}5.23 \\
4.66 \\
2.74(-2) \\
1.01(-7) \\
8.23(-11)\end{array}$ & $\begin{array}{l}5.25 \\
4.70-4.72 \\
2.04(-2) \\
\ldots \\
\ldots\end{array}$ \\
\hline $\mathrm{SO}_{2} \ldots$ & $\begin{array}{l}271 \\
265 \\
180 \\
100\end{array}$ & $\begin{array}{l}0.290 \\
0.210 \\
2.13(-4) \\
6.65(-12)\end{array}$ & $\begin{array}{l} \\
\ldots \\
\ldots\end{array}$ \\
\hline $\mathrm{PH}_{3} \ldots$ & $\begin{array}{r}298 \\
273 \\
267 \\
180 \\
100 \\
80\end{array}$ & $\begin{array}{l}15.4 \\
1.75 \\
0.958 \\
7.29(-3) \\
1.62(-8) \\
1.06(-11)\end{array}$ & $\begin{array}{r}16.0 \\
1.6 \\
1.0\end{array}$ \\
\hline
\end{tabular}

NoTE. $-a(-b)=a \times 10^{-b} . P_{\text {experimental }}$ is data from sources in Table 1. Values in braces for $\mathrm{CH}_{4}$ assume amorphous water ice as coexisting phase (see text).
We now use our fitted parameters to derive dissociation pressures of clathrate containing guest molecules for which few or no laboratory data are available. We reason in the following manner: the mixed force parameters $c_{m}, \varepsilon_{m}, \rho_{m}$, fitted to laboratory data, characterize the averaged intermolecular potential interaction between guest molecule and the cage comprised of water molecules. Similarly shaped guest molecules will likely experience similar types of interactions with the cage; hence, using mixing rules we can derive Kihara parameters for the unknown molecule from those of a similar type of molecule for which dissociation pressure data exist. We use the so-called geometric mean and hard-sphere mixing rules (Prausnitz 1969, pp. 63, 104):

$$
c_{m}=c, \quad \varepsilon_{m}=\left(\varepsilon \varepsilon_{\omega}\right)^{1 / 2}, \quad \rho_{m}=\frac{\rho+\rho_{\omega}}{2}
$$

where the unsubscripted parameters refer to the pure guest molecules, and $\omega$ refers to the water lattice. We take $c_{\omega}=0$ as in Holder, Corbin, and Papadopoulos (1980), since the main guest-water molecule interaction is with the pointlike oxygen atom; guest-hydrogen atom interaction has been shown by numerical modeling to be much less important (Tse, Klein, and $\mathrm{McD}$ Donald 1983). Values of $\varepsilon, \rho$, and $c$ are obtained from tables based on virial data (e.g., Hirschfelder, Curtiss, and Bird 1954, pp. 1110-1112); these along with the mixed parameters for the known clathrate are used to derive effective $\rho_{\omega}, \varepsilon_{\omega}$ from equations (9). Using tabulated $\varepsilon, \rho, c$ for the unknown molecule, equations (9) give the mixed parameters for this species from which we derive dissociation pressure in equations (1)-(3) and (7) or (8). Because two-parameter L-J fits to virial data are better established as "reference data" in the literature, and our two-parameter fit to the known $\mathrm{N}_{2}$ dissociation pressures is fairly accurate, we employed the L-J potential for this portion of the study.

We first used argon to derive dissociation pressures for neon and helium, two molecules of cosmochemical interest. To test out our procedure we derived $\mathrm{Kr}$ and $\mathrm{Xe}$ dissociation pressures from the argon data and compared them with the fits from laboratory data. The results are shown in Figure 3. The xenon data pertain to structure I clathrate, although our model indicates dissociation pressures in both structures to be quite similar. We have inserted the molecular parameters from Xe data in the structure II model to compare against results based on the argon data. As shown in Figure 3, the correspondence for $\mathrm{Kr}$ is entirely satisfactory over a wide range of temperatures. The Xe pressures derived from argon data are $10-10^{4}$ times higher than those fitted to Xe data. This is due to the large collision diameter of the xenon atom, comparable to the size of the small cage, which dominates the dissociation pressure in structure II (but not I). Our model is thus not valid in this casé; from a computational stance small and physically insignificant changes in $\rho_{m}$ for Xe result in large changes in dissociation pressure. Since we are predicting pressures for $\mathrm{He}$ and $\mathrm{Ne}$, which have smaller collision diameters than $\mathrm{Ar}$, this is of no concern, and the $\mathrm{Kr}$ results validate the procedure. When applied to $\mathrm{He}$ and $\mathrm{Ne}$, the curves in Figure 4 result. Of course, the pressures shown are far outside the ideal gas regime, and so must properly be regarded as fugacities. The figure illustrates that these gases are extremely poor clathrate 


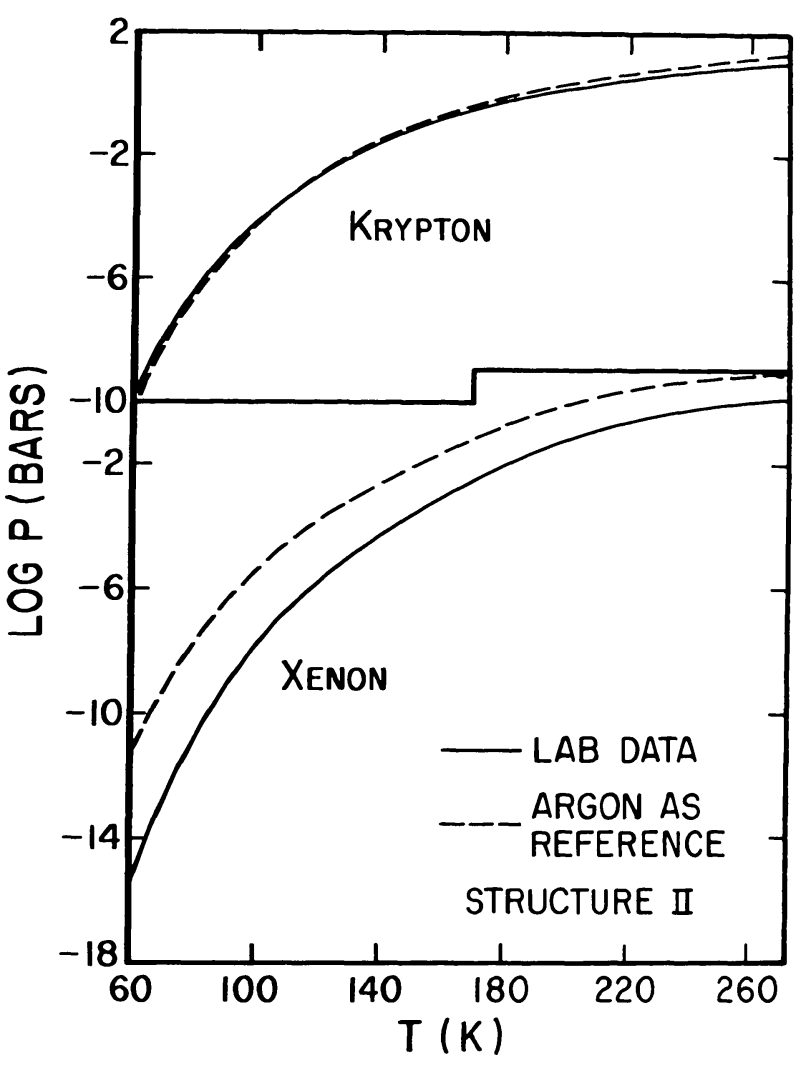

FIG. 3.-Temperature vs. $\log$ of the dissociation pressure for krypton (top) and xenon (bottom) clathrates. Solid lines calculated by adjusting molecular parameters $\varepsilon$ and $\rho$ to fit laboratory data for the two clathrates; dashed lines calculated from argon clathrate data and mixing rules for molecular parameters.

formers, because their interaction with the cage is exceedingly weak. This is also the origin of the unusual shape of the He curve: $\mathrm{C}$ is an extremely weak function of $T$, and the term $\Delta \mu^{\beta} / T$ dominates the $P(T)$ dependence.

We next derive carbon monoxide and molecular hydrogen clathrate dissociation pressures from those of molecular nitrogen. CO may have been the predominant form of carbon in the solar nebula (Lewis and Prinn 1980); $\mathrm{H}_{2}$ would be the most abundant constituent in any scenario of satellite or planet formation. Here we use a two-parameter L-J fit to the measured $\mathrm{N}_{2}$ clathrate dissociation pressures assuming a structure II model; to test our procedure we derive mixed parameters for $\mathrm{O}_{2}$ as plotted in Figure 5. The agreement with the scant laboratory data (about $30 \%$ too low) is not as good as in the noble gas case. We thus have less confidence in these fits; however, the molecular weight of $\mathrm{CO}$ and hence pure L-J parameters are closer to $\mathrm{N}_{2}$ than are those of $\mathrm{O}_{2}$, so we expect our procedure to be more valid for $\mathrm{CO}$ than $\mathrm{O}_{2}$. The same cannot be said for $\mathrm{H}_{2}$, and we must consider the derived dissociation pressures to be of qualitative interest only.

Figure 4 shows the result for $\mathrm{H}_{2}$, and Figure 5 the result for $\mathrm{CO}$ clathrate. The similarity in dissociation pressures for $\mathrm{N}_{2}$

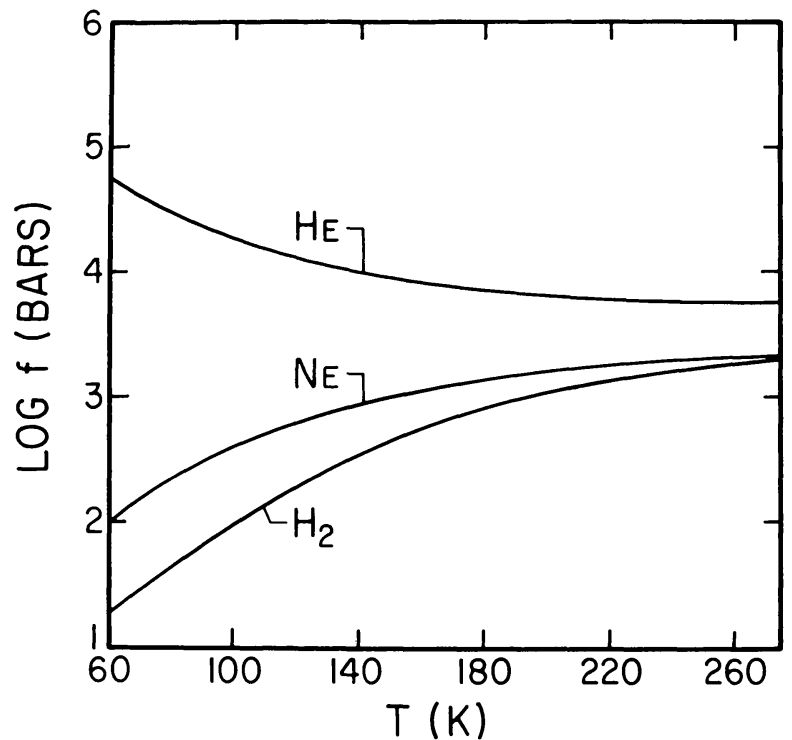

FIG. 4.-Dissociation fugacity vs. temperature for helium, $\mathrm{H}_{2}$, and neon clathrates predicted from the theory.

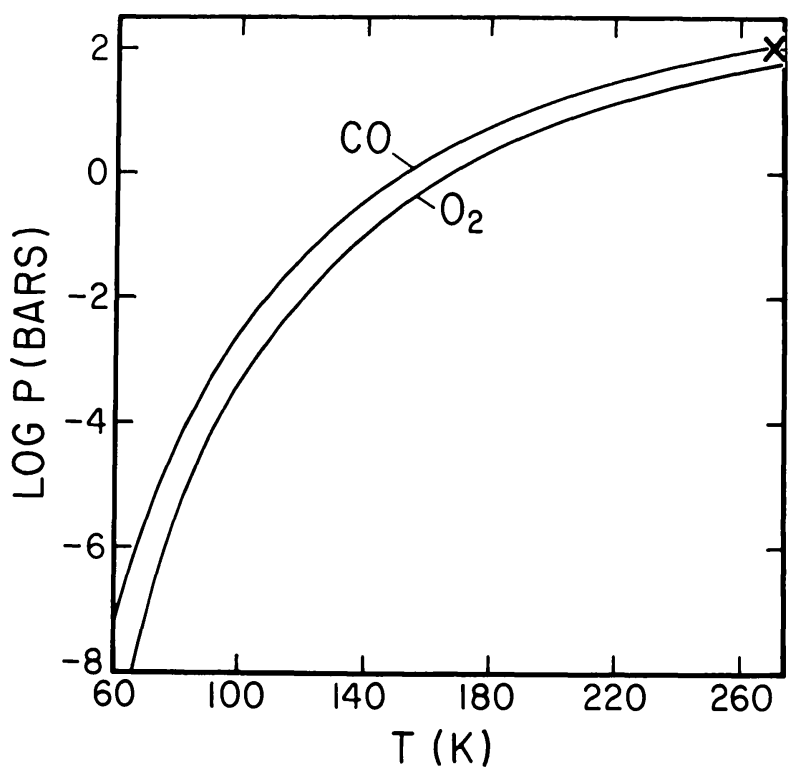

Fig. 5.-Dissociation pressure vs. temperature for $\mathrm{CO}$ and $\mathrm{O}_{2}$ clathrates predicted from theory. Cross marks $\mathrm{O}_{2}$ data at $\sim 272 \mathrm{~K}$ from van Cleef and Diepen (1965). The $\mathrm{N}_{2}$ dissociation pressure line would be essentially coincident with that for $\mathrm{CO}$ on this scale.

and $\mathrm{CO}$ structure II clathrates are striking, within a factor of 2 even down at $T=60 \mathrm{~K}$. CO structure I dissociation pressures are $\sim 20 \%$ lower than those for structure II. We thus conclude that $\mathrm{CO}$ and $\mathrm{N}_{2}$ are very similar in their ability to incorporate into clathrate, with the tentative suggestion that $\mathrm{CO}$ dissociation pressures are slightly lower than those of $\mathrm{N}_{2} . \mathrm{H}_{2}$ dissocia- 
tion pressures are extremely high, with a temperature dependence expected for a very small molecule with a low value of $\varepsilon$ (weak energy of interaction). Thus the derived values of $P$ serve as a qualitative indicator that $\mathrm{H}_{2}$, by itself, is a poor clathrate "former." The small size of $\mathrm{H}_{2}$ along with its ubiquity in astrophysical settings raises the possibility that $\mathrm{H}_{2}$ could occupy a cage already occupied by another guest molecule. We present a model for such double occupancy below; before doing so we return to $\mathrm{CO}$ and examine the effect of its dipole moment on dissociation pressure.

The weak dipole moment of $\mathrm{CO}$ ( 0.1 debyes; Prausnitz 1969 , p. 64) distinguishes it from the nonpolar $\mathrm{N}_{2}$. Although a residual, fluctuating dipole field may be produced by the clathrate cage (Davidson 1971), the induction force generated on nonpolar guest molecules is accounted for in the force parameters derived from experimental data. The interaction should be similar for similar molecules-hence, our bootstrap procedure for deriving force constants for one guest species from that of others. The polar nature of $\mathrm{CO}$ implies that an additional dipole-dipole interaction between $\mathrm{CO}$ and the cage field must be incorporated in the potential. There are two opposing effects: (1) the additional attractive term lowers the dissociation pressure, and (2) at low temperature the $\mathrm{CO}$ dipole may orient, restricting the rotational degrees of freedom and raising the dissociation pressure. The first effect is straightforward to calculate. The interaction potential energy between the $\mathrm{CO}$ dipole moment $\xi_{\mathrm{CO}}$ and that of another dipole $\xi_{p}$, averaged over all orientations, is (Prausnitz 1969, p. 57)

$$
\bar{\Gamma}_{i j}=-\frac{\frac{2}{3} \xi_{\operatorname{CO}}^{2} \xi_{p}^{2}}{r^{6} k_{\mathrm{B}} T}
$$

The $\mathrm{CO}$ molecule interacts with a variable cage averaged dipolar field estimated by Davidson (1971) to be roughly comparable to that of a single water molecule. To examine the effect of equation (10) we use a somewhat large value of $\xi_{p}=2 \xi_{\mathrm{H}_{2} \mathrm{O}}$, and plot in Figure $6 P^{\prime} / P$, the ratio of $\mathrm{CO}$ clathrate dissociation pressure including the dipolar term to that given in Figure 5. The Boltzmann factor in $\bar{\Gamma}_{i j}$ produces the strong temperature dependence. The weakness of the $\mathrm{CO}$ dipole means that $P^{\prime}$ differs appreciably from $P$ only at $T \lesssim 100 \mathrm{~K}$. For $\xi_{p}=\xi_{\mathrm{H}_{2} \mathrm{O}}, P^{\prime} \approx P$ down to $60 \mathrm{~K}$. Gurikov (1983) states that the numerical factor in equation (10) should be $\frac{1}{3}$; in any event the equation is not strictly appropriate for this situation, as noted by D. W. Davidson, and is used to illustrate the effect only.

Opposing this stabilization of $\mathrm{CO}$ clathrate is the possible loss of one or both rotational degrees of freedom of the $\mathrm{CO}$ molecule at low temperatures due to the oriented nature of the cage dipolar field. Infrared spectra of ethylene oxide structure I clathrate suggests that reorientation of the guest becomes strongly inhibited at low ( 50-100 K) temperatures (Bertie and Jacobs 1977). If the excitation of rotational degrees of freedom of the encaged molecule is less than that of a free molecule, then $z<1$ but $\omega(r)$ must necessarily be larger (eq. [3]). The net effect should always be increased stability of the clathrate (i.e., lower dissociation pressure) since if it were not more stable, the guest molecule would spontaneously revert to

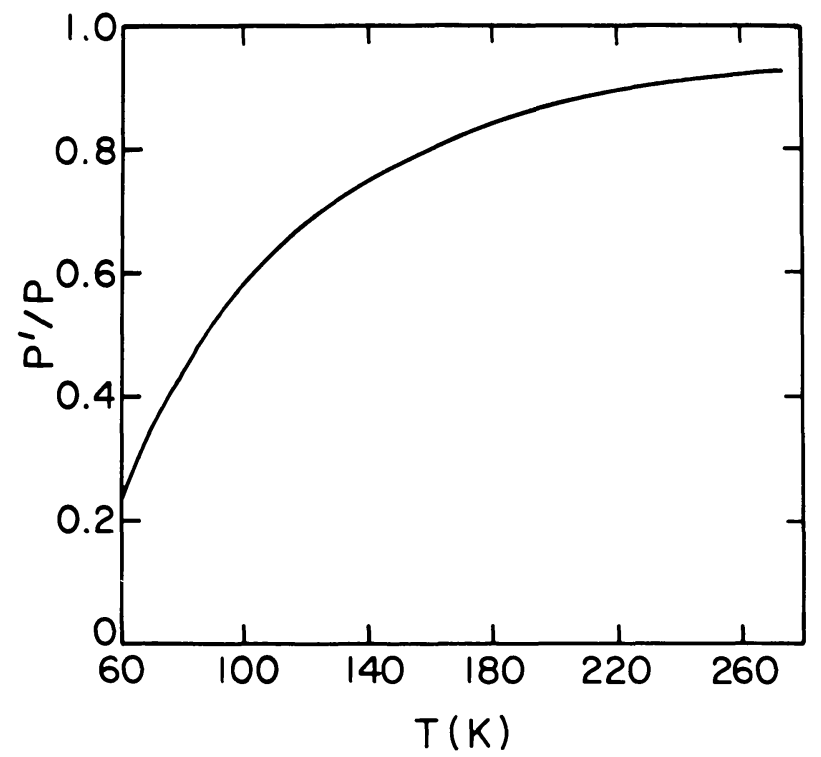

FIG. 6. - Ratio of CO clathrate dissociation pressures for two models of the CO-cage interaction, plotted as a function of temperature. The quantity $P$ is the dissociation pressure assuming dispersion and cage dipole-CO induced dipole interactions; $P^{\prime}$ includes $\mathrm{CO}$ dipole interaction with dipole field of cage.

the freely rotating state with a release of energy (cf. Mazo 1964). In reality, the situation is somewhat more complex since the parameters in the potential have been adjusted to fit data that may include some of the effects of rotational inhibition. The net effect of hindered rotation cannot be accurately calculated, therefore.

However, we can get a rough measure of the possible magnitude of the effect from the Helmholtz free energy difference $\Delta F$ between the nonrotating molecule and the freely rotating diatomic molecule:

$$
\Delta F \approx R T \ln \left(\frac{i I k_{\mathrm{B}} T}{\bar{h}^{2}}\right)-\Delta E-R T \ln N
$$

(Landau and Lifshitz 1969, pp. 115 and 132), where $I=$ moment of inertia of the molecule, $\bar{h}=$ Planck's constant divided by $2 \pi, N=$ number of cage sites per unit cell $(\sim 8)$, $\Delta E=$ energy gained over spherically averaged state by oriented guest molecule, $i=1$ for like, 2 for unlike, nuclei. Using $\Delta E=180 \mathrm{cal} \mathrm{mole}^{-1}$ derived for chlorine (Davidson 1971), we find $\Delta F$ is negative (clathrate stabilized by hindrance) for $T \lesssim 110 \mathrm{~K}$ for $\mathrm{N}_{2}$ and $\lesssim 70 \mathrm{~K}$ for $\mathrm{CO}$. Even where $\Delta F$ is positive, it is small compared with the energy of encagement (Barrer and Edge 1967) because of cancellation of terms.

We conclude that the dissociation pressure of $\mathrm{CO}$ clathrate is similar to, but less than, that of $\mathrm{N}_{2}$ clathrate. At $60 \mathrm{~K}$ the ratio of $\mathrm{CO}$ to $\mathrm{N}_{2}$ clathrate dissociation pressures could be as low as $\frac{1}{20}$ but is probably closer to $\frac{1}{4}$. 


\section{d) Double Occupancy of Cage Sites}

We now consider the possibility of double occupancy of cage sites by small molecules (suggested by van der Waals and Platteeuw 1959); in particular, methane-molecular hydrogen double occupancy. Neon and helium are only briefly considered, the former because of its low solar abundance relative to hydrogen (Anders and Ebihara 1982) and the latter because of its exceedingly low Langmuir constants, even compared with $\mathrm{H}_{2}$. In calculating the importance of this effect for the situation of a gaseous nebula around the Sun or a giant planet, we anticipate somewhat the result that the degree of incorporation of a guest molecule in clathrate relative to other guests is directly proportional to the relative abundance in the coexisting gas. Also, for simplicity, we use a square-well cage potential (Prausnitz 1969, p. 109) in the model which follows.

Consider a molecule with Langmuir constant $C_{M}$ occupying the large cage of a structure I clathrate, and a second molecule with Langmuir $C_{\mathrm{H}} \ll C_{M}$. Equation (10) can be generalized to read (writing out the large-cage term only)

$$
\begin{aligned}
\frac{\Delta \mu^{\beta}}{k T}=\frac{3}{23} \ln ( & 1+C_{M} P_{M}+C_{\mathrm{H}} P_{\mathrm{H}}+q_{M \mathrm{H}} C_{M} C_{\mathrm{H}} P_{M} P_{\mathrm{H}} \\
& \left.+\frac{1}{2} q_{M M} C_{M}^{2} P_{M}^{2}+\frac{1}{2} q_{\mathrm{HH}} C_{\mathrm{H}}^{2} P_{\mathrm{H}}^{2}\right)
\end{aligned}
$$

where $P_{M}, P_{\mathrm{H}}$ are the partial pressures of $M$ and $\mathrm{H}$ in the gas, and $q_{i j}$ gives the probability of double occupancy of a cage site by molecules $i$ and $j$. Note that in the van der Waals and Platteeuw theory $q_{i j}=0$ by assumption. Here it is estimated as

$$
q_{i j}=1-\frac{V_{E, i}}{V_{\text {cage }}},
$$

where $V_{\text {cage }}=4 \pi / 3\left(a-\sigma_{j}\right)^{3}$ is the volume available for occupation of the cage by molecule $j$ and $V_{E, i}$ is the excluded volume due to occupation by $i$, given by the common volume of spheres of radius $a-\sigma_{j}$ and $\sigma_{i}$. The quantity $\sigma_{j}$ is the square-well collision diameter for molecule $j$ 's interaction with $\mathrm{H}_{2} \mathrm{O}$ molecules in the cage, while $\sigma_{i}$ is the value for molecule $i$ 's interaction with molecule $j$. Recall $\sigma^{6}=\rho^{6} / 2$. Figure 7 illustrates the intersection. In employing this model we have made the somewhat artificial assumption that one molecule is fixed at the center of the cage while the other is forced to occupy other regions in the cage. This introduces some asymmetry in the results for two different molecules (i.e., $q_{\mathrm{H} M} \neq q_{M \mathrm{H}}$ ) but is adequate for our purposes.

Evaluating the volume of the region of intersection of the two spheres, we find

$$
\begin{array}{r}
V_{E, i}=\pi\left\{\left(a-\sigma_{j}\right)^{3}\left[\frac{2}{3}-\cos \theta_{2}+\frac{1}{3} \cos ^{3} \theta_{2}\right]\right. \\
\left.+\sigma_{i}^{3}\left[\frac{2}{3}-\cos \theta_{1}+\frac{1}{3} \cos ^{3} \theta_{1}\right]\right\}, \\
\theta_{1}=\pi / 2-\theta_{2} / 2, \quad \theta_{2}=2 \sin ^{-1} \frac{\sigma_{i}}{2\left(a-\sigma_{j}\right)} .
\end{array}
$$

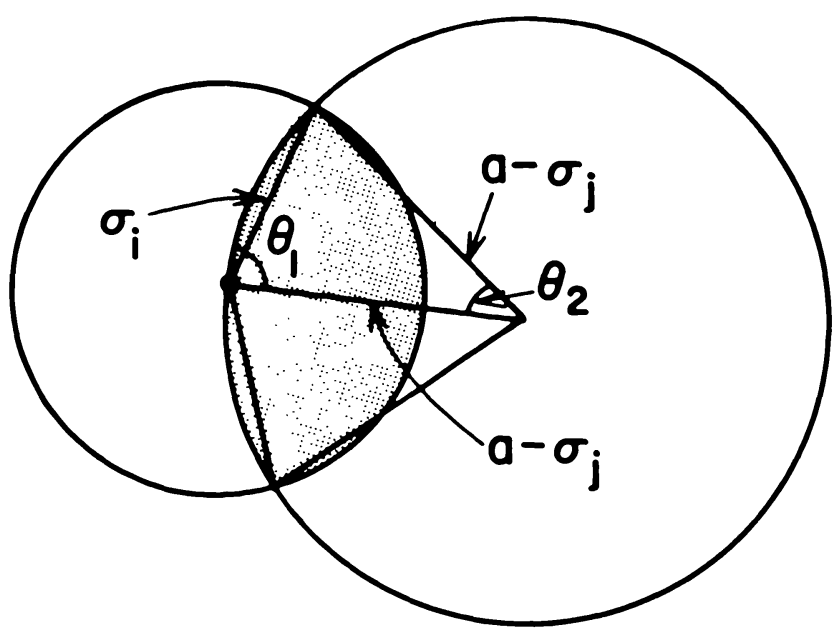

FIG. 7.-Geometric construction for clathrate double-occupancy calculation. The quantities $\sigma_{i}$ and $\sigma_{j}$ are the collision diameters for molecules $i$ and $j, a$ is cage radius; the shaded region is the cage volume excluded from molecule $j$ given by the common volume of spheres of radius $a-\sigma_{j}$ and $\sigma_{i}$.

Using square-well parameters estimated for $\mathrm{H}_{2}\left(\sigma_{\mathrm{H}}\right)$ and $\mathrm{CH}_{4}$ $\left(\sigma_{M}\right)$ from data in Hirschfelder $(1954$, pp. 160, 552, 1110), we find for the structure I large cage $q_{M M}=0, q_{\mathrm{HH}} \sim 0.4-0$, $\bar{q}_{\mathrm{H} M} \sim 0.04-0$, where $\bar{q}_{\mathrm{H} M}$ is an average of the two permutations. For the structure II large cage $q_{M M}=0, q_{\mathrm{HH}} \sim 0.6-0$, $\bar{q}_{\mathrm{H} M} \sim 0.2-0$. The range in $q$ reflects uncertainty in the choice of square-well parameters for these molecules; in particular, the value of $\sigma$ for the guest molecule cage interaction. If the low value of $\sigma_{w}$ in McKoy and Sinanoglu (1963) is used, we get the upper value of $q$. If $\sigma_{w}$ derived in our fits is used, we find $q=0$. Although we prefer our fits and choice of $\Delta \mu^{\beta}$ over that of McKoy and Sinanoglu, we cannot a priori rule out their selection in this calculation, nor can we rule out distortion of the cage structure to accommodate $\mathrm{H}_{2}$. We apply the double-occupancy model to clathrate composition in primordial nebulae in § VII.

\section{CLATHRATE EQUILIBRIA AT MODERATE PRESSURES IN THE PRESENCE OF AMMONIA}

We now turn to the problem of clathrate equilibria in the presence of ammonia. Prinn and Fegley (1981) concluded that in protosatellite nebulae, the abundance of $\mathrm{NH}_{3}$ should be comparable to or much greater than that of $\mathrm{N}_{2}$. At nebular gas $\left(\mathrm{H}_{2}\right)$ pressures of 0.1 bars, if most of the nitrogen is in the form of ammonia, then the latter will condense out as a stoichiometric hydrate, $\mathrm{NH}_{3} \cdot \mathrm{H}_{2} \mathrm{O}$, at $130 \mathrm{~K}$. Clearly, then, Titan and perhaps the small Saturnian satellites (Stevenson 1982 $a$; Ellsworth and Schubert 1983) may have incorporated large quantities of ammonia, perhaps as much as $15 \%$ of the total ice budget. We wish to determine what effect ammonia could have on clathrate formation in nebular and higherpressure environments. In this section we calculate equilibria between the ammonia hydrates and clathrate, and between clathrate, water ice, and an ammonia-water solution. These relationships are displayed on a single slice of an $\mathrm{NH}_{3}-\mathrm{H}_{2} \mathrm{O}-\mathrm{CH}_{4}$ phase diagram (Fig. 8). Here we restrict 
ourselves to moderate pressures for which pressure corrections to the fugacities of the various components are negligible or of secondary importance; in the next section we present a model for clathrate equilibria (including the presence of ammonia) for pressures in excess of a kilobar.

The phase diagram for the ammonia-water system at moderate pressures is fairly well established (see, e.g., Clifford and Hunter 1933; Rollet and Vuillard 1956; Chan and Giauque 1964; Tsiklis, Linshits, and Goryunova 1965; Lewis 1969; van Kasteren 1973; Miller 1974). The last reference contains an excellent depiction of the phase diagram, which therefore will not be reproduced here. It is characterized by three eutectics, at $\sim 33,57$, and 80 mole percent ammonia, defining minimum melting points between the solids $\mathrm{H}_{2} \mathrm{O}, \mathrm{NH}_{3} \cdot \mathrm{H}_{2} \mathrm{O}$, $2 \mathrm{NH}_{3} \cdot \mathrm{H}_{2} \mathrm{O}$, and $\mathrm{NH}_{3}$. Because the solar $\mathrm{N}$-to-O ratio is $\sim 0.13$, the low-ammonia eutectic (at 33\%) is particularly relevant to solar system applications; its minimum melting point is $172 \mathrm{~K}$, the coexisting solids being pure water ice and $\mathrm{NH}_{3}-\mathrm{H}_{2} \mathrm{O}$ ("ammonia hydrate"). We neglect the presence of an $\mathrm{NH}_{3} \cdot 2 \mathrm{H}_{2} \mathrm{O}$ solid for two reasons. First, its domain of stability in equilibrium with the solution is very small (Rollet and Vuillard 1956); hence, the chemical potential of the dihydrate is only slightly less than that of the monohydrate plus water ice. Data on specific heats and entropies of the phases (Giauque and Stout 1936; Dorsey 1940; Hildenbrand and Giauque 1953; Chan and Giauque 1964) indicate this to be true down to temperatures of less than $100 \mathrm{~K}$. The dihydrate is more difficult to make in the laboratory than the monohydrate (Fink and Sill 1982). We conclude that under planetary formation conditions monohydrate plus water ice could condense out in preference to dihydrate. Second, if dihydrate did condense out in the primordial gas, a roughly two- to fourfold excess in $\mathrm{CH}_{4}$ gas pressure over the clathrate dissociation pressure would be sufficient to cause conversion to monohydrate plus methane clathrate. This conclusion is based upon a simple calculation analogous to one presented below for the monohydrate in a $\mathrm{CH}_{4}$ gas, but should be regarded as an estimate because of the lack of data on the vapor pressure of the dihydrate. Behavior of the $\mathrm{NH}_{3} \cdot \mathrm{H}_{2} \mathrm{O}$ melting curve at high ( $>$ kilobar) pressures is under investigation by Johnson, Schwake, and Nicol (1985). A rough extrapolation of the eutectic temperature to high pressures is given in Hunten et al. (1984).

At low temperatures and pressures in the presence of ammonia, a "competition" would be expected to ensue between the methane, which seeks water ice so as to form clathrate, and ammonia, which would like to form a stoichiometric hydrate. At higher temperatures and pressures, the liquid ammonia-water field would also be expected to affect the stability of the coexisting clathrate, since the energy cost in converting liquid water to clathrate hydrate structure differs from that of water ice and results in a different slope to the dissociation pressure-temperature curve.

In what follows we make the following assumptions: (1) $\mathrm{NH}_{3}$ does not incorporate as a guest molecule in clathrate, and (2) $\mathrm{NH}_{3}$ does not incorporate in the cage-forming ice clathrate lattice, nor does it form a clathrate lattice structure of its own. Although no experiments relevant to these assumptions have been made, finite occupancy of cages for (1) by
$\mathrm{NH}_{3}$ molecules is required by statistical mechanics, but this is likely to be a small effect in most situations of interest, where the $\mathrm{NH}_{3}$ partial pressure is very low because of the formation of a stoichiometric ammonia hydrate. The Langmuir constant for $\mathrm{NH}_{3}$ occupancy of the large cage was estimated by including contributions from dispersion forces and dipole interaction between $\mathrm{NH}_{3}$ and the cage dipole moment (Davidson 1971). Although the Langmuir constant is large compared with that for $\mathrm{CO}$ or $\mathrm{CH}_{4}$ (because of the dipole interaction), the fugacity of $\mathrm{NH}_{3}$ in the $\mathrm{NH}_{3}-\mathrm{H}_{2} \mathrm{O}$ solution is low so that even at room temperature $\mathrm{NH}_{3}$ occupies a small fraction of cages compared with $\mathrm{CO}$ or $\mathrm{CH}_{4}$. (2) is more difficult to justify, but the greater dipole moment of $\mathrm{H}_{2} \mathrm{O}$ than that of $\mathrm{NH}_{3}$ and the high propensity for water hydrogen bonding suggest that cages would be composed primarily of water molecules.

The above assumptions allow us to equate chemical potentials for the ammonia hydrates and clathrate and evaluate them using properties known from laboratory data or calculated from § III. Since complete clathrate and hydrate formation would exhaust the available water ice in a solar composition nebula, we examine phase equilibria between the following assemblages:

(a) $\mathrm{NH}_{3}-\mathrm{H}_{2} \mathrm{O}_{(\mathrm{L})}+\mathrm{CH}_{4(\mathrm{FL})}$,

(b) $\mathrm{NH}_{3}-\mathrm{H}_{2} \mathrm{O}_{(\mathrm{L})}+\mathrm{CH}_{4} \cdot 6 \mathrm{H}_{2} \mathrm{O}+\mathrm{CH}_{4(\mathrm{FL})}$,

(c) $\mathrm{NH}_{3}-\mathrm{H}_{2} \mathrm{O}_{(\mathrm{L})}+$ ice $\mathrm{I}+\mathrm{CH}_{4(\mathrm{FL})}$,

(d) $\mathrm{NH}_{3} \cdot \mathrm{H}_{2} \mathrm{O}_{(\mathrm{s})}+\mathrm{ice} \mathrm{I}+\mathrm{CH}_{4(\mathrm{FL})}$,

(e) $\mathrm{NH}_{3} \cdot \mathrm{H}_{2} \mathrm{O}(\mathrm{s})+\mathrm{CH}_{4} \cdot 6 \mathrm{H}_{2} \mathrm{O}+\mathrm{CH}_{4(\mathrm{FL})}$,

(f) $2 \mathrm{NH}_{3} \cdot \mathrm{H}_{2} \mathrm{O}_{(\mathrm{s})}+\mathrm{CH}_{4} \cdot 6 \mathrm{H}_{2} \mathrm{O}+\mathrm{CH}_{4(\mathrm{FL})}$.

Here $(\mathrm{s})=$ solid, $(\mathrm{L})=$ liquid, $(\mathrm{FL})$ refers to vapor, liquid, or supercritical phase as appropriate, $\mathrm{CH}_{4} \cdot 6 \mathrm{H}_{2} \mathrm{O}$ indicates structure I methane clathrate, and ice I is pure hexagonal water ice I. The effect of methane solubility in the liquid ammonia-water is neglected as it is small under these conditions (Culberson 1951; Wiebe and Gaddy 1937); it is examined more carefully in $\S \mathrm{V}$.

We deal first with equilibria between $(b)$ and $(c)$, and $(d)$ and $(e)$, and restrict ourselves for the moment to an ammonia mole fraction relative to water of $15 \%$ ( solar). In the corresponding water-ammonia system, at $T$ less than $249 \mathrm{~K}$, water ice forms with consequent enrichment of the liquid in ammonia until the eutectic at $172 \mathrm{~K}$ is reached, at which point ammonia hydrate freezes out in coexistence with water ice, the ratio of the two being $\sim 0.18$. The methane pressure at which clathrate forms is dictated by the equilibria given in equations (1) and (2). Since the ammonia-water solution is in coexistence with pure water ice, the additional condition

$$
\mu_{\mathrm{H}_{2} \mathrm{O}}^{\mathrm{L}}=\mu_{\mathrm{H}_{2} \mathrm{O}}^{\mathrm{I}}
$$

holds, where $\mu_{\mathrm{H}_{2} \mathrm{O}}^{\mathrm{L}}$ is the chemical potential of water in the ammonia-water solution and $\mu_{\mathrm{H}_{2} \mathrm{O}}^{\mathrm{I}}$ that in ice $\mathrm{I}$. With the additional constraint that the solubility of methane in ammonia-water solid or liquid is negligible, the equilibria between clathrate, methane vapor, and water ice is identical with the pure water case, and hence the dissociation pressure of methane clathrate is unchanged. The abundance of clathrate is then limited by the amount of water ice in coexistence with 
the ammonia-water solution. The same is true below the 172 $\mathrm{K}$ eutectic, where $85 \%$ of the water is available for clathration.

Thermodynamics also predicts the formation of clathrate from water in the ammonia-water solution and even from the ammonia hydrate. We deal with the latter case first (i.e., assemblages $[e]$ and $[f]$, no free water ice present); the equilibrium is essentially

$$
\begin{aligned}
& \mathrm{CH}_{4}(\mathrm{FL})+12\left(\mathrm{NH}_{3} \cdot \mathrm{H}_{2} \mathrm{O}\right)(\mathrm{s}) \\
& \quad \Leftrightarrow \mathrm{CH}_{4} \cdot 6 \mathrm{H}_{2} \mathrm{O}+6\left(2 \mathrm{NH}_{3} \cdot \mathrm{H}_{2} \mathrm{O}\right)(\mathrm{s})+\mathrm{CH}_{4}(\mathrm{FL}) .
\end{aligned}
$$

In terms of chemical potentials,

$$
\mu_{2 \mathrm{NH}_{3}-\mathrm{H}_{2} \mathrm{O}}+\mu_{\mathrm{H}_{2} \mathrm{O}}^{\mathrm{cl}}=2 \mu_{\mathrm{NH}_{3}-\mathrm{H}_{2} \mathrm{O}}
$$

Now,

$$
\begin{aligned}
\mu_{\mathrm{NH}_{3}-\mathrm{H}_{2} \mathrm{O}} & =\mu_{\mathrm{NH}_{3}}^{\mathrm{v}}+\mu_{\mathrm{H}_{2} \mathrm{O}}^{\mathrm{v}} \\
& =k T\left(\ln \frac{P_{\mathrm{NH}_{3}}^{\mathrm{NH}_{3}-\mathrm{H}_{2} \mathrm{O}}}{P_{\mathrm{NH}_{3}}^{R}}+\ln \frac{P_{\mathrm{H}_{2} \mathrm{O}}^{\mathrm{NH}_{3}-\mathrm{H}_{2} \mathrm{O}}}{P_{\mathrm{H}_{2} \mathrm{O}}^{R}}\right)
\end{aligned}
$$

and

$$
\mu_{2 \mathrm{NH}_{3}-\mathrm{H}_{2} \mathrm{O}}=k T\left(2 \ln \frac{P_{\mathrm{NH}_{3}}^{2 \mathrm{NH}_{3}-\mathrm{H}_{2} \mathrm{O}}}{P_{\mathrm{NH}_{3}}^{R}}+\ln \frac{P_{\mathrm{H}_{2} \mathrm{O}_{3}}^{2 \mathrm{NH}_{3}-\mathrm{H}_{2} \mathrm{O}}}{P_{\mathrm{H}_{2} \mathrm{O}}^{R}}\right),
$$

where $\mu_{x}^{\mathrm{v}}=$ chemical potential in the vapor phase of constituent $x, P_{x}^{y}=$ vapor pressure of constituent $x$ over solid compound $y$, and $P_{x}^{R}$ is a reference pressure for the pure component $x$ at the system temperature $T$. Now let

$$
\mu_{\mathrm{H}_{2} \mathrm{O}}^{\mathrm{cl}}-\mu_{\mathrm{H}_{2} \mathrm{O}}^{\mathrm{I}}=\Delta \mu^{0}-\nu k T \ln \left(C_{\mathrm{CH}_{4}} P_{\mathrm{CH}_{4}}\right),
$$

where $C_{\mathrm{CH}_{4}} P_{\mathrm{CH}_{4}} \gg 1, \Delta \mu^{0}$ is the chemical potential difference between the empty clathrate and ice I, and for the purposes of this rough analysis we combine both cages of the clathrate ( $\nu=4 / 23$ ) and let $C$ be the Langmuir constant of the large cage. This will give a lower bound to the pressure at which reaction (16) occurs. Also

$$
\mu_{\mathrm{H}_{2} \mathrm{O}}^{\mathrm{I}}=k T \ln \frac{P_{\mathrm{H}_{2} \mathrm{O}}^{\mathrm{I}}}{P_{\mathrm{H}_{2} \mathrm{O}}^{R}} .
$$

Combining equations (17), (18), (19), (20), and (21) and canceling out the reference pressures,

$$
\begin{aligned}
\nu k T \ln \frac{P_{\mathrm{CH}_{4}}^{0}=}{P_{\mathrm{CH}_{4}}} & k T \ln \frac{\left(P_{\mathrm{H}_{2} \mathrm{O}}^{\mathrm{NH}_{3}-\mathrm{H}_{2} \mathrm{O}} P_{\mathrm{NH}_{3}}^{N_{3}-\mathrm{H}_{2} \mathrm{O}}\right)^{2}}{\left(P_{\mathrm{NH}_{3}}^{\left.2 \mathrm{NH}_{3}-\mathrm{H}_{2} \mathrm{O}\right)^{2}}\right.} \\
& \times \frac{1}{P_{\mathrm{H}_{2} \mathrm{O}}^{2 \mathrm{NH}_{3}-\mathrm{H}_{2} \mathrm{O} P_{\mathrm{H}_{2} \mathrm{O}}^{\mathrm{I}}}},
\end{aligned}
$$

where we have substituted for $\Delta \mu^{0}$ the expression $\nu k T \ln \left(C_{\mathrm{CH}_{4}} P_{\mathrm{CH}_{4}}^{0}\right)$, where $P_{\mathrm{CH}_{4}}^{0}$ is the methane clathrate dissociation pressure in coexistence with pure water ice. Note also $C_{\mathrm{CH}_{4}} P_{\mathrm{CH}_{4}}^{0}$ and $C_{\mathrm{CH}_{4}} P_{\mathrm{CH}_{4}} \gg 1$ at temperatures of interest.

To evaluate the vapor pressures of the ammonia and water constituents we use the expressions of Haudenschild (1970), who relied on Lewis (1969) as the data source. Evaluating equation (22) using his expression yields the result

$$
P_{\mathrm{CH}_{4}}=P_{\mathrm{CH}_{4}}^{0} 10^{(0.618+50.54 / T)(1 / \nu)} \text {. }
$$

Above this methane pressure $\mathrm{NH}_{3} \cdot \mathrm{H}_{2} \mathrm{O}$ is converted to methane clathrate and $2 \mathrm{NH}_{3} \cdot \mathrm{H}_{2} \mathrm{O}$. The methane pressure as a function of temperature is plotted in Figure 8, for which the ammonia-to-water ratio is $15 \%$. The line defined by equation (23) separates regions I and II as indicated on the figure. Note that for the given temperature range this pressure is above the saturation vapor pressure for methane (indicated by the line separating regions II and III); $P_{\mathrm{CH}_{4}}$ is thus strictly the fugacity of the $\mathrm{CH}_{4}$ liquid at which the conversion occurs. An external pressure would have to be applied to raise the fugacity to the appropriate value for the conversion to occur, violating our assumption in this section that $P V$ effects are not important.

Extended to lower temperatures appropriate to outer solar system conditions, the phase boundary pressure is $3.1 \times 10^{-3}$ bars at $60 \mathrm{~K}$. Recalling that this is a lower bound (if we consider only large-cage occupancy, $P_{\mathrm{CH}_{4}}=2.0$ bars), we conclude that this transition is not of interest to planetary or satellite formation scenarios, since the methane itself condenses out as a solid at $10^{-4}$ bars and $60 \mathrm{~K}$. Thus, although the conversion of $\mathrm{NH}_{3} \cdot \mathrm{H}_{2} \mathrm{O}$ to $2 \mathrm{NH}_{3} \cdot \mathrm{H}_{2} \mathrm{O}$ in the presence of methane is an interesting possibility which merits laboratory investigation, at present we see no application of this transformation to solar system objects. Also, the transformation

$$
6\left(2 \mathrm{NH}_{3} \cdot \mathrm{H}_{2} \mathrm{O}\right)+\mathrm{CH}_{4(\mathrm{~g})} \Leftrightarrow 12 \mathrm{NH}_{3}+\mathrm{CH}_{4} \cdot 6 \mathrm{H}_{2} \mathrm{O}
$$

should occur at even higher pressures and hence is not considered here.

We now consider phase equilibrium between assemblages (a) and (b), i.e., clathrate formation in an ammonia-water solution in coexistence with methane vapor, no free water ice being present. The equilibrium in this case is

$$
\mu_{\mathrm{H}_{2} \mathrm{O}}^{\mathrm{cl}}=\mu_{\mathrm{H}_{2} \mathrm{O}}^{\mathrm{NH}_{3}-\mathrm{H}_{2} \mathrm{O}_{(\mathrm{L})}} \text {, }
$$

so that $\Delta \mu^{\beta}$, the free energy difference between the empty hydrate cage and the coexisting water phase, is now $\mu_{\mathrm{H}_{2} \mathrm{O}}^{\beta}-$ $\mu \mathrm{NH}_{3}-\mathrm{H}_{2} \mathrm{O}$.

Since we are now considering the liquid-water field, $\Delta h(T)$ in equation (5) is dominated by the enthalpy of freezing, $1440 \mathrm{cal} \mathrm{mole}^{-1}$. Using values from Holder, Corbin, and Papadopoulos (1980) and ignoring the pressure term, equation (5) becomes

$$
\begin{aligned}
\Delta \mu^{\beta}(T) & =\Delta h^{\beta}+\frac{T}{T_{f}}\left[\Delta \mu^{\beta}\left(T_{0}\right)-\Delta h^{\beta}\right] \\
& =-1161+1471 \frac{T}{T_{f}} .
\end{aligned}
$$




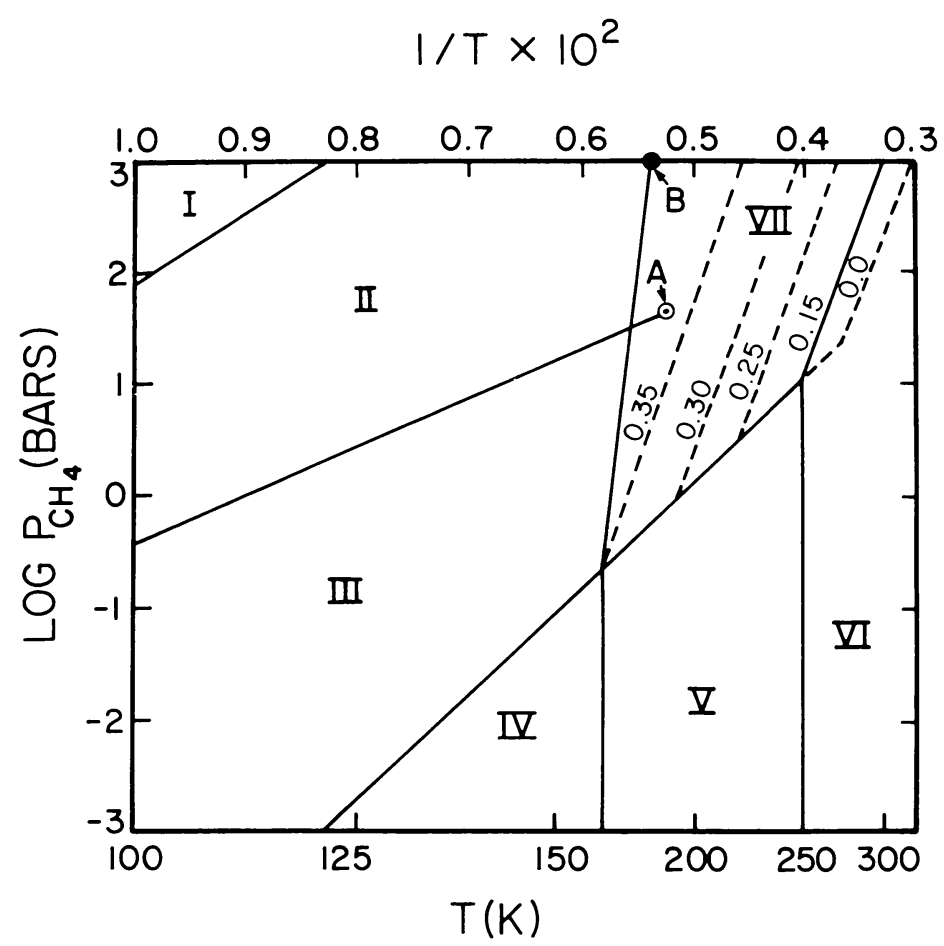

FIG. 8. - Phase diagram for methane clathrate in the presence of ammonia, plotted as methane partial pressure vs. temperature. Within regions I, II, and part of VII the ordinate must be redefined as methane fugacity. The $\mathrm{NH}_{3}$ mole fraction $x_{a}$ (relative to ammonia + water) is 0.15 . Roman numerals correspond to regions in which the following phases are stable: I: $2 \mathrm{NH}_{3} \cdot \mathrm{H}_{2} \mathrm{O}_{(\mathrm{s})}+\mathrm{CH}_{4} \cdot 5.75 \mathrm{H}_{2} \mathrm{O}+\mathrm{CH}_{4(\mathrm{~L})} ; \mathrm{II}: \mathrm{NH}_{3} \cdot \mathrm{H}_{2} \mathrm{O}_{(\mathrm{s})}+\mathrm{CH}_{4} \cdot 5.75 \mathrm{H}_{2} \mathrm{O}+\mathrm{CH}_{4(\mathrm{~L})}$; III: $\mathrm{NH}_{3} \cdot \mathrm{H}_{2} \mathrm{O}_{(\mathrm{s})}+\mathrm{CH}_{4} \cdot 5.75 \mathrm{H}_{2} \mathrm{O}+\mathrm{CH}_{4(\mathrm{v})}$; IV: $\mathrm{NH}_{3} \cdot \mathrm{H}_{2} \mathrm{O}_{(\mathrm{s})}+$ ice I $+\mathrm{CH}_{4(\mathrm{v})} ; \mathrm{V}: \mathrm{NH}_{3}-\mathrm{H}_{2} \mathrm{O}_{(\mathrm{L})}+$ ice I $+\mathrm{CH}_{4(\mathrm{FL})} ; \mathrm{VI}: \mathrm{NH}_{3}-\mathrm{H}_{2} \mathrm{O}_{(\mathrm{L})}+\mathrm{CH}_{4(\mathrm{FL})} ; \mathrm{VII}$ : $\mathrm{NH}_{3}-\mathrm{H}_{2} \mathrm{O}_{(\mathrm{L})}+\mathrm{CH}_{4} \cdot 5.75 \mathrm{H}_{2} \mathrm{O}+\mathrm{CH}_{4(\mathrm{FL})}$. Here $\mathrm{s}=$ solid, $\mathrm{v}=$ vapor, $\mathrm{L}=$ liquid, $\mathrm{FL}=$ fluid, and $\mathrm{CH}_{4} \cdot 5.75 \mathrm{H}_{2} \mathrm{O}=\mathrm{CH}_{4}$ clathrate but does not necessarily imply complete occupancy of cage sites. Point A defines $\mathrm{CH}_{4}$ critical point, and B defines the $\mathrm{NH}_{3}-\mathrm{H}_{2} \mathrm{O}$ freezing point for a $40 \%$ ammonia-water solution. Dashed lines are phase boundaries between regions VI and VII for ammonia fractions (relative to water + ammonia) other than 0.15 .

For the ammonia-water case, $T_{f}$ is the freezing temperature of water ice in the mixture. Freezing points versus ammonia mole fraction are given in Haudenschild (1970). An alternative but equivalent expression for $\Delta \mu^{\beta}$ is

$$
\Delta \mu^{\beta}(T)=\Delta h^{\beta}+\frac{T}{T_{f}^{0}}\left[\Delta \mu^{\beta}\left(T_{0}\right)-\Delta h^{\beta}\right]-k T \ln \gamma X_{\mathrm{H}_{2} \mathrm{O}},
$$

where $T_{f}^{0}$ is now the freezing point of pure water, $X_{\mathrm{H}_{2} \mathrm{O}}$ is the mole fraction of water in solution, and $\gamma$ is the activity coefficient of the ammonia-water solution.

Inserting equation (24) into equation (1), and using Langmuir constants derived in $\S \mathrm{III}$, the methane clathrate dissociation pressure in equilibrium with a $15 \%$ ammonia-water solution is plotted as a function of temperature in Figure 8 as the solid line separating regions VI and VII. This line terminates by intersecting the dissociation pressure curve for the water-ice case at the water-ice freezing point of the ammonia-water solution, $249 \mathrm{~K}$. A vertical line is drawn downward from this point, separating region $\mathrm{V}$ with $\mathrm{NH}_{3}-\mathrm{H}_{2} \mathrm{O}$ solution containing water ice from VI with no water ice. Another vertical line is drawn at $172 \mathrm{~K}$, where the $33 \% \mathrm{NH}_{3}$ eutectic is reached, below which temperature (IV) ammonia hydrate and water ice exist, and above which (V) water ice coexists with ammonia-water solution. Since $P<P_{\text {dissociation }}$ for regions IV, V, and VI, the methane is a vapor or supercritical fluid.

In region VII we have plotted clathrate dissociation pressures for ammonia mole fractions in excess of $15 \%$. These plotted lines terminate at the dissociation pressure curve for the water-ice case at the appropriate water-ice freezing line for the labeled concentrations. Also, the pure water case is plotted by extending the dissociation pressure curve for the water-ice case to $273 \mathrm{~K}$, and using equation (24) with $T_{f}=T_{f}^{0}$. These dotted curves may be thought of as projections of the third dimension of the diagram, the $X_{\mathrm{H}_{2} \mathrm{O}}$ coordinate, onto the $X_{\mathrm{H}_{2} \mathrm{O}}=0.85$ slice. They define region VII in their own slice to be smaller $\left(X_{\mathrm{H}_{2} \mathrm{O}}<0.85\right)$ or larger $\left(X_{\mathrm{H}_{2} \mathrm{O}}>0.85\right)$ than in the displayed slice. Since in region VII the clathrate is produced at the expense of water in solution with ammonia, for a given temperature and methane partial pressure one may read off the concentration of the ammonia-water solution in equilibrium with the clathrate and, hence, calculate how much clathrate is produced.

Finally, we plot the boundary between regions II and VII, and III and VII, at which $\mathrm{NH}_{3} \cdot \mathrm{H}_{2} \mathrm{O}$ in coexistence with the ammonia-water solution freezes out. The line is constructed by calculating the clathrate dissociation pressure for solutions with $X_{\mathrm{H}_{2} \mathrm{O}}<0.66$, at a temperature corresponding to the $\mathrm{NH}_{3} \cdot \mathrm{H}_{2} \mathrm{O}$ freezing point for the given liquid composition. 
For our pressure restrictions, the highest practical ammonia concentration is $40 \%\left(X_{\mathrm{H}_{2} \mathrm{O}}=0.6\right)$. To calculate the clathrate dissociation pressure at this composition a hypothetical standard state, that of water ice freezing out of the solution, was assumed. The use of hypothetical standard states is a common "trick" in fluid-phase equilibrium studies (see Prausnitz 1969, pp. 386-387).

The quantities $\Delta \mu^{\beta}$ and $P_{\text {diss }}$ were evaluated for the solution at the appropriate $\mathrm{NH}_{3} \cdot \mathrm{H}_{2} \mathrm{O}$ freezing point calculated from Haudenschild (1970) and yielded 950 bars at $T=185 \mathrm{~K}$, plotted as point B on Figure 8. A line was then drawn from B down to the eutectic at $172 \mathrm{~K}$ on the water ice-clathrate dissociation pressure line, to define the entire $\mathrm{NH}_{3} \cdot \mathrm{H}_{2} \mathrm{O}$ freezing boundary. Although we have used low-pressure data to define point B, work by Johnson, Schwake, and Nicol (1985) indicates that the $\mathrm{NH}_{3} \cdot \mathrm{H}_{2} \mathrm{O}$ freezing temperature shifts upward by only a few degrees from 1 to 1000 bars.

The graph is terminated at $10^{3}$ bars to avoid serious modification to the diagram from pressure effects, considered in the next section. Even so, at $\gtrsim 10^{2}$ bars the dissociation pressure lines in VII and VI should bend slightly toward the left with increasing pressure; i.e., the dissociation pressure increases more rapidly with temperature than the figure indicates.

\section{HIGH-PRESSURE EQUILIBRIA}

Calculation of clathrate stability at high pressures requires consideration of several effects neglected in previous sections: (1) the volume increase in conversion of ice or liquid water to the empty hydrate cage begins to dominate the corresponding free energy change above a few hundred bars; (2) the fugacity of the guest molecule phase in coexistence with the clathrate is no longer the partial pressure and must be explicitly calculated in terms of the system temperature and total pressure; (3) compression of the cage sites at high pressure alters the interaction potential between guest and host molecules. In this section we present our model for incorporating these effects, display the $P-T$ phase diagram for methane (and molecular nitrogen) clathrate stability in coexistence with pure water and water-ammonia, and consider the effect of methane solubility in water. Application of our results to satellite interiors is made in $\S$ VII.

\section{a) Formulation for Pure Water Case}

The equations of clathrate equilibrium valid at high pressure have been given in $\S$ III. In constructing the high-pressure diagram, it is useful to derive a Clausius Clapeyron relation, $d P / d T$, from equations (1) and (2). Differentiating with respect to $T, P$,

$$
\begin{aligned}
\frac{d \Delta \mu^{\beta}}{d T} d T & +\frac{d \Delta \mu^{\beta}}{d P} d P \\
= & k\left[\nu \ln (1+C f)+\frac{\nu T}{1+C f}\left(C \frac{d f}{d T}+f \frac{d C}{d T}\right)\right] d T \\
& +\frac{\nu k T}{1+C f} \frac{C d f}{d P} d P
\end{aligned}
$$

where we consider a single guest species and for brevity write down terms for only a single cage - the addition of the second is trivial. We have also assumed $f d C / d P \ll C d f / d P$; this is justified later in this section. Superscripts on quantities referring to the guest molecule and its bulk phases are implicit; superscript $\beta$ refers to differences between empty clathrate cage and coexisting water ice or liquid phase. Using standard thermodynamic relations, for the $\mathrm{H}_{2} \mathrm{O}$,

$$
d \Delta \mu^{\beta}=\Delta V^{\beta}(P, T) d P-\Delta S^{\beta}(P, T) d T,
$$

and for the guest species,

$$
\begin{aligned}
& \left.d\left(k T \ln \frac{f}{f^{0}}\right)\right|_{P}=-\left(S-S^{0}\right) d T=k \ln \frac{f}{f^{0}} d T+\frac{k T}{f} \frac{d f}{d T} d T \\
& \left.d(k T \ln f)\right|_{T}=V d P=\frac{k T}{f} \frac{d f}{d P} d P
\end{aligned}
$$

where $f^{0}, S^{0}$ are fugacity and entropy of a reference state, taken to be in the ideal gas regime of the guest molecule. Solving for $d f / d T$ and $d f / d P$ in equation (28), plugging these and equation (27) into equation (26), and rearranging, we find

$$
\begin{aligned}
\frac{d P}{d T}= & k \llbracket \nu \ln (1+C f) \\
& +T\left\{\frac{\left[\left(S^{0}-S\right)-k \ln f / f^{0}\right] f}{k T} \frac{C \nu}{1+C f}+\frac{d C}{d T} \frac{\nu f}{1+C f}\right\} \\
& +\frac{\Delta S^{\beta}}{k} \rrbracket\left(\Delta V^{\beta}-f V \frac{C \nu}{1+C f}\right)^{-1}
\end{aligned}
$$

for all applications in this section the quantity $C f \gg 1$, so

$$
\begin{aligned}
\frac{d P}{d T}= & \left\{\nu\left[k \ln C f+\left(S^{0}-S\right)-k \ln \frac{f}{f^{0}}+\frac{k T}{C} \frac{d C}{d T}\right]+\Delta S^{\beta}\right\} \\
& \times\left(\Delta V^{\beta}-V \nu\right)^{-1}
\end{aligned}
$$

Comparing equations (27) and (24) we see, for structure I clathrate,

$$
\begin{aligned}
& \Delta S^{\beta}=-\frac{\Delta \mu^{\beta}\left(T_{f}, P_{0}\right)-\Delta h^{\beta}\left(P_{0}\right)}{T_{f}} \\
& =\left\{\begin{array}{ll}
0 & \left(T<T_{f}\right) \\
-\frac{1471}{T_{f}} \mathrm{cal} \mathrm{mole}^{-1} \mathrm{~K}^{-1} & \left(T>T_{f}\right)
\end{array} .\right.
\end{aligned}
$$

Examination of equation (30) confirms the qualitative picture given in Figure 2. Consider first the ideal gas regime. Here $\left(S^{0}-S\right)=k \ln f / f^{0}$, and for $T<T_{f}, \Delta S^{\beta}=0$; then the numerator becomes $\nu k \ln C P+\nu(k T / C)(d C / d T)$. With $d C / d T<0$, inserting values for $C, P$, we find the numerator to be negative. The denominator is the difference (per mole of $\mathrm{H}_{2} \mathrm{O}$ ) between the volume expansion required to form a cage from the coexisting water phase, and the volume of the coexisting guest phase weighted by the number of cage sites 
per water molecule. It is, in effect, the net volume gained or lost by converting 1 mole of ice (or liquid water) plus gas into clathrate. Since in the ideal gas state $V>\Delta V^{\beta} / \nu$, the denominator is negative, and $d P / d T$ is positive, as the dissociation pressure increases with temperature. Inserting numerical values for methane reproduces the slope of the dissociation pressure curve derived in $\S$ III for $T<T_{f}$. For $T>T_{f}, \Delta S^{\beta}$ is negative, and $d P / d T$ increases. However, as $P$ increases into the kilobar regime, $V$ decreases sharply, while $\Delta V^{\beta}$ increases or decreases only slightly (see numerical evaluations below). Thus $\Delta V^{\beta}-V \nu \rightarrow 0$ and $d P / d T \rightarrow \infty$. At $\Delta V^{\beta}=V \nu, d P / d T$ $=\infty$ and the curve of dissociation pressure versus temperature shows a maximum. For higher pressures $d P / d T$ becomes negative, its absolute magnitude decreasing now with increasing pressure. All terms in the numerator are important throughout the pressure range; numerical evaluation (see below) indicates that the numerator remains less than zero throughout this regime. Eventually the dissociation pressure curve crosses the liquid-water-high-pressure ice phase boundary, and $\Delta S^{\beta}$ is again assumed zero. It is not truly zero, because the enthalpy change in going from ice I to the relevant high-pressure phases is nonzero (Eisenberg and Kauzmann 1969 , p. 95). It is, however, much smaller than the ice-to-liquid enthalpy change and, hence, is neglected in equation (30). The numerator drops to a small value, and with a large value for the denominator, $d P / d T$ becomes small; i.e., as $T$ decreases, $P$ remains fairly constant.

We calculate the phase diagram explicitly first for pure water, and $\mathrm{CH}_{4}$ as the structure $\mathrm{I}$ guest molecule, since it likely was the primary clathrate former incorporated in Titan. The effect of pressure dependence of the Langmuir constant and the solubility of methane in water will then be considered, followed by inclusion of an additional clathrate former, $\mathrm{N}_{2}$. We then calculate the methane clathrate phase diagram with ammonia present.

We construct the phase diagram by evaluating equations (1) and (2) along an isothermal path in pressure. The methane fugacity $f$ given by these equations is compared with the fugacity of the coexisting pure methane phase $f_{c}$ at each pressure, which is calculated along $T-P$ paths shown in Figure 9. Where $f_{c}>f$, the clathrate is stable. Equation (30) is also used to determine the slope of the high-pressure phase boundary at each temperature point since uneven quality of the data results in some scatter of this boundary at various temperatures. Calculation of $\Delta \mu^{\beta}$ and $f_{c}$ is detailed in the Appendix.

Figure 10 displays the completed phase diagram for the water-methane system. At both the low- and high-pressure ends, in the water-ice field, the magnitude of the slope $d P / d T$ of the stability boundaries is small. A discontinuity in slope at the solid-fluid methane phase boundary (drawn from Grace and Kennedy 1967) is expected, but is a rather small effect. The computed change in slope and increase in $|d P / d T|$ at the water liquid-ice boundary is seen clearly at the high-pressure end; a similar change at the low-pressure end is present as well. The precise pressure value at which $d P / d T=\infty$ was found by setting the denominator of equation (30) equal to zero, and the corresponding temperature (which is the maximum temperature at which methane clathrate is stable) was

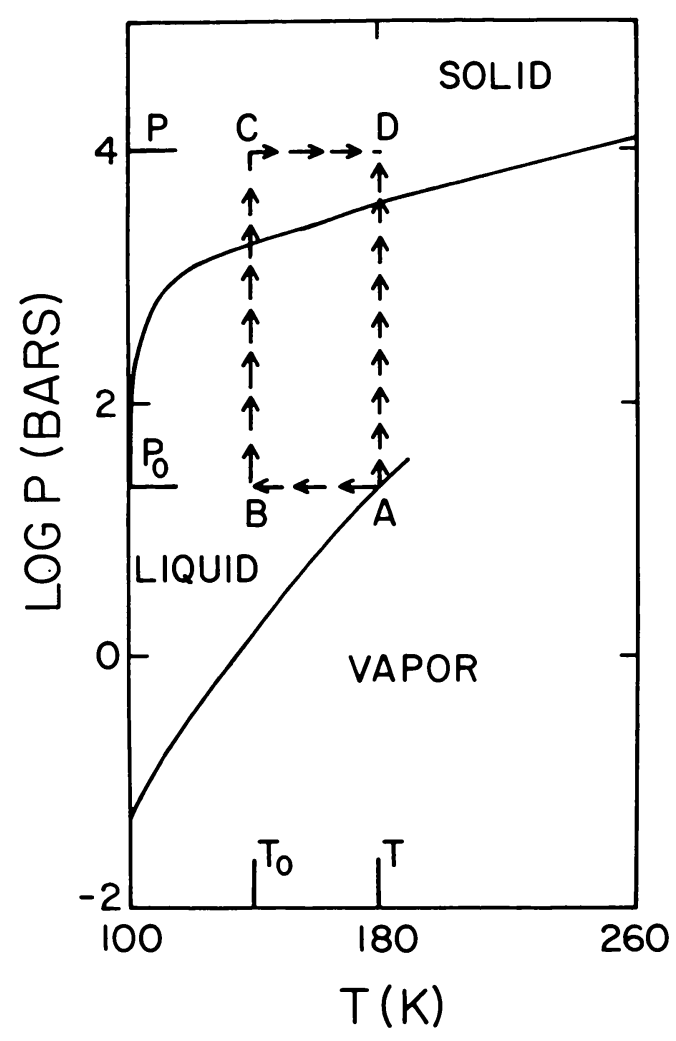

FIG. 9.-Schematic phase diagram of methane, plotted as pressure vs. temperature, illustrating thermodynamic paths taken to calculate fugacity. Beginning at point $\mathrm{A}$ on the liquid-vapor boundary, fugacity at $\mathrm{D}$ was calculated by integrating along $\mathrm{A}-\mathrm{D}$, as well as along $\mathrm{A}-\mathrm{B}-\mathrm{C}-\mathrm{D}$.

found by extrapolating the data of Marshall, Saito, and Kobayashi (1964).

Work by Tammann and Krige (1925) on the high-pressure stability of $\mathrm{SO}_{2}$ clathrate, as described by Davidson (1973), confirms the general shape of the left-hand portion of Figure 10. At a mechanical pressure of $\sim 2$ kilobars, they found that the $\mathrm{SO}_{2}$ clathrate decomposition temperature hit a maximum with respect to pressure. Van Berkum and Diepen (1979) find the critical point to be at 3.1 kilobars. At the critical pressure the volume change in going from $\mathrm{SO}_{2}$ vapor + ice to clathrate was roughly zero, the same criterion by which we found the maximum clathrate stability temperature for the $\mathrm{CH}_{4}-\mathrm{H}_{2} \mathrm{O}$ system. Comparable high-pressure data for $\mathrm{CH}_{4}, \mathrm{CO}$, or $\mathrm{N}_{2}$ clathrate do not exist in the literature. (The Marshall, Saito, and Kobayashi 1964 data do not extend to sufficiently high pressure to show the $d P / d T=\infty$ point by itself.) Data for pure xenon clathrate (Aaldijk 1971) up to 3.7 kilobars do not reach a critical point.

So far we have neglected the effect of pressure on the Langmuir constant $C$. To determine the effect of cage compression we assume that the decrease in volume of the clathrate with pressure is taken up by a corresponding decrease in cage volume. We then calculate the Langmuir constant for the 


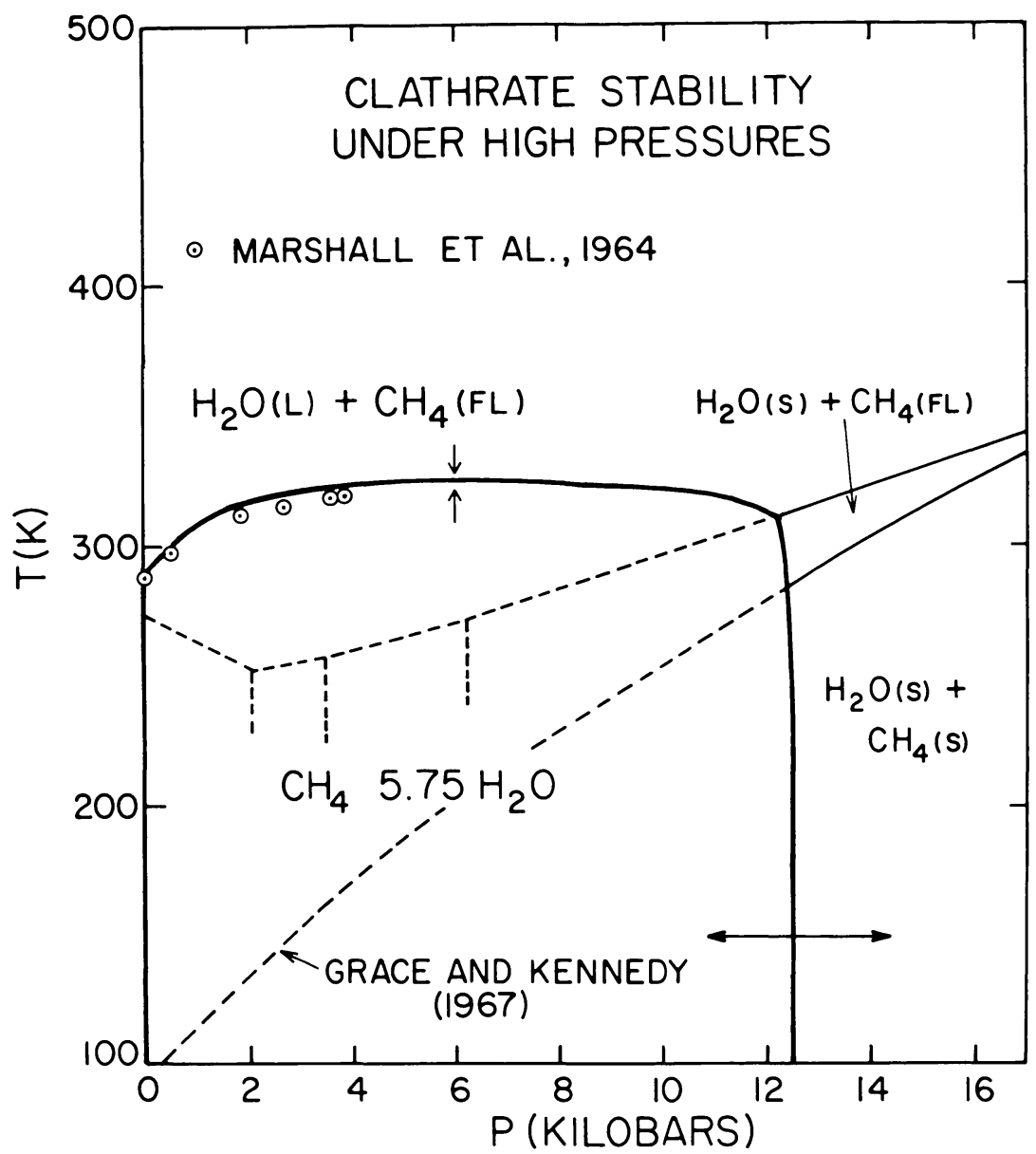

FIG. 10. - Phase diagram of methane clathrate plotted as temperature vs. pressure. Calculated phase boundary between clathrate and water + methane (assuming zero solubility of methane in water) is given by heavy solid line; data of Marshall, Saito, and Kobayashi (1964) are plotted as $\odot$. Vertical arrows indicate estimated critical point; horizontal arrows at 12.5 kilobars show uncertainty in calculated high-pressure boundary. Light solid lines are solid-liquid boundaries for water and methane. They are extended into the clathrate stable region but are only relevant there if one of the components (water or methane) is completely depleted in forming clathrate. The low-pressure end of the clathrate stability field is indistinguishable from the left-hand axis on this scale.

large and small cages for cage radii corresponding to the compression. Using $K^{\beta}=10^{-5} \mathrm{bar}^{-1}$, the decrease in both cage radii at 10 kilobar is $\approx 3.7 \%$. Figure 11 plots Langmuir constants for both structure I cages as a function of cage radius at $273 \mathrm{~K}$. The effect is of opposite sign for the large versus small cages. Methane is a sufficiently small molecule that a modest decrease in the large-cage radius increases the attractive portion of the guest-host potential without bringing the molecules so close that the repulsive portion of the potential becomes dominant as in the small cage.

Using our formulation of the high-pressure case, we solve for the high-pressure phase boundary using the altered Langmuir constants and find that it changes by $\sim 1 \%$ from the value based on the low-pressure Langmuir constants. Numerical tests confirm the general insensitivity of the phase boundary $P$ to modest changes in $C$, as can be seen by inspecting eq. [1].) The behavior of the Langmuir constants for $\mathrm{N}_{2}$ (and hence $\mathrm{CO}$ ) as a function of cage radius is similar to that for $\mathrm{CH}_{4}$, as expected since these molecules are similar in size.

Very large guest molecules, such as $\mathrm{SO}_{2}$, may be squeezed out of the cage at rather small reductions in cage radii. Using the fitted Kihara parameters for $\mathrm{SO}_{2}$, derived in $\S \mathrm{III}$, we find that at low pressures occupancy of the small cage is not possible, whereas large-cage occupancy is very favorable. A $5 \%$ reduction in large-cage size results in a decrease of $C$ by a factor of $10^{6}$, effectively preventing $\mathrm{SO}_{2}$ from incorporating at all. Although the high-pressure phase diagram of $\mathrm{SO}_{2}$ clathrate is not worked out here (see discussion above of experimental data of Tammann and Krige 1925), such a calculation would have to take $d C / d P$ into account.

\section{b) Effect of Methane Solubility in Liquid Water}

We now consider the effect of methane solubility in liquid water. We assume in what follows that the solubility of 


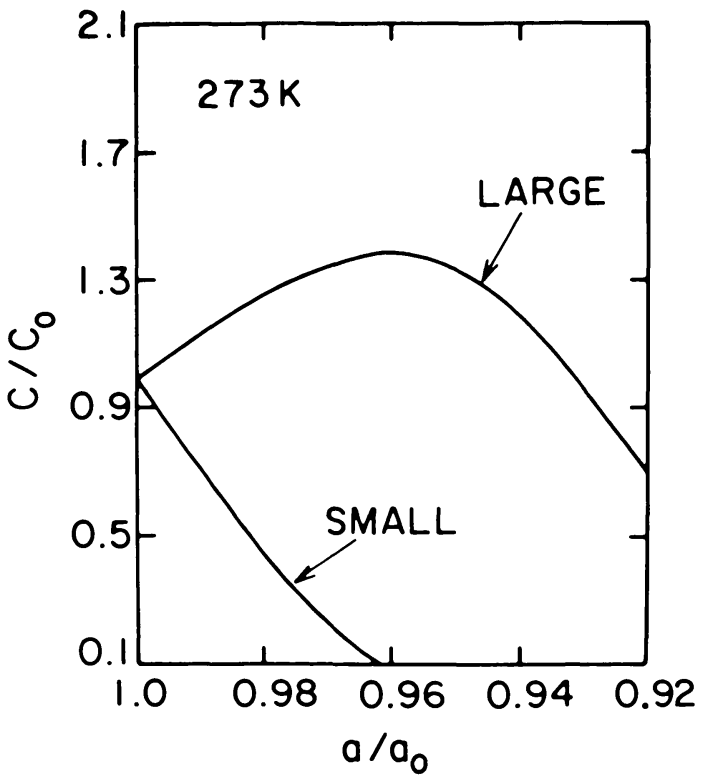

FIG. 11.-Langmuir constants for methane clathrate as a function of cage radius at $273 \mathrm{~K}$, for large and small cages, normalized to zero pressure values.

methane in water ice is zero, and that the solubility of water ice in the coexisting methane phase is negligible (Rebiai, Rest, and Scurlock 1983). Two effects of importance are considered: (1) the presence of a saturated solution of methane in liquid water lowers the chemical potential of the liquid-water phase in coexistence with the clathrate, effectively destabilizing the clathrate; (2) stability of clathrate is possible in an unsaturated methane-water solution for which no coexisting pure methane phase is present.

Experimental data on solubility of $\mathrm{CH}_{4}$ in $\mathrm{H}_{2} \mathrm{O}$ extend up to $\sim 680$ bars at temperatures down to $298 \mathrm{~K}$ (Culberson 1951). At $310 \mathrm{~K}$ and 680 bars, the mole fraction of methane in liquid water, $x_{2}$, is $7.8 \times 10^{-3}$. We use a modified Henry's law which assumes that the fugacity of solute 2 in liquid solvent 1 is proportional to the mole fraction of 2 in 1 (Prausnitz 1969, p. 335):

$$
f_{2}^{L}=H_{2,1}(T, P) x_{2}(T, P),
$$

valid over some limited range of $x_{2}$.

Using thermodynamic relations in Prausnitz (1969, pp. 30, 357 ), we find

$$
\begin{aligned}
x_{2}\left(T_{0}, P\right)= & \frac{P_{0}}{H_{2,1}\left(T_{0}, P_{0}\right)} \\
& \times \exp \left\{\frac{1}{k T_{0}} \int_{P_{0}}^{P}\left[V\left(T_{0}, P\right)-\bar{V}^{\infty}\left(T_{0}, P\right)\right] d P\right\} \\
& \times \exp \left\{-\frac{A\left(T_{0}, P_{0}\right)}{k T_{0}}\left[\left(1-x_{2}\right)^{2}-1\right]\right\}
\end{aligned}
$$

where $P_{0}$ is a reference pressure in the ideal gas regime, $H_{2,1}\left(T_{0}, P_{0}\right)$ is the Henry's law constant for methane dissolved in water at $T_{0}$ and $P_{0}, V$ is the volume of the coexisting pure methane phase, $\bar{V}^{\infty}$ is the volume of methane dissolved in $\mathrm{H}_{2} \mathrm{O}$ at infinite dilution, and $A$ is an empirical parameter which characterizes the difference between the $\mathrm{H}_{2} \mathrm{O}-\mathrm{CH}_{4}$ interaction and the $\mathrm{H}_{2} \mathrm{O}-\mathrm{H}_{2} \mathrm{O}$ interaction. Equation (33) is written in slightly different form in Prausnitz (1969, p. 360). The quantity $V\left(T_{0}, P\right)$ is found as described in $\S \mathrm{V} a, P_{0}$ is chosen to be 300 bars, and $H_{2,1}\left(T_{0}, 300\right)$ is taken from Culberson (1951). Since the effect of $A$ is small up to $x_{2} \sim 0.1$, the uncertainty in other parameters makes it impossible to estimate $\boldsymbol{A}$ from the Culberson data. Based on data on $\mathrm{N}_{2}$ in $\mathrm{NH}_{3}$, an analogous system consisting of a nonpolar solute in polar solvent, we estimate $A$ to be negative (net repulsion) and approximately one-fourth the heat of vaporization of $\mathrm{H}_{2} \mathrm{O}$. The quantity $\bar{V}^{\infty}$ is equally difficult to estimate. The Culberson data up to 600 bars are consistent with $\bar{V}^{\infty}$ fitted to a linear function of pressure such that it is $35 \mathrm{~cm}^{3} \mathrm{~mole}^{-1}$ at 300 bars and $30 \mathrm{~cm}^{3} \mathrm{~mole}^{-1}$ at 10 kilobars. The latter value is especially uncertain. Data in Shmulovich et al. (1980) on excess volume of mixing in the $\mathrm{H}_{2} \mathrm{O}-\mathrm{CH}_{4}$ system indicate that the system approaches a critical point above 1 kilobar pressure and $700 \mathrm{~K}$ temperature, where the excess volume of mixing is small $\left(\leq 5 \mathrm{~cm}^{3} \mathrm{~mole}^{-1}\right)$. However, the excess volume at temperatures of relevance to the present study is not given, and so extrapolation of the Culberson data to derive $\bar{V}^{\infty}$ is necessary.

Our results are given in Figure 12 at $310 \mathrm{~K}$. They indicate a low solubility of $5 \times 10^{-3}$ mole fraction with a small increase from 300 to 10,000 bars, followed by a sharp increase. The behavior of the curve is governed by the ability of the several exponential terms to cancel one another; since two of the terms are poorly known, the curve must be regarded as preliminary.

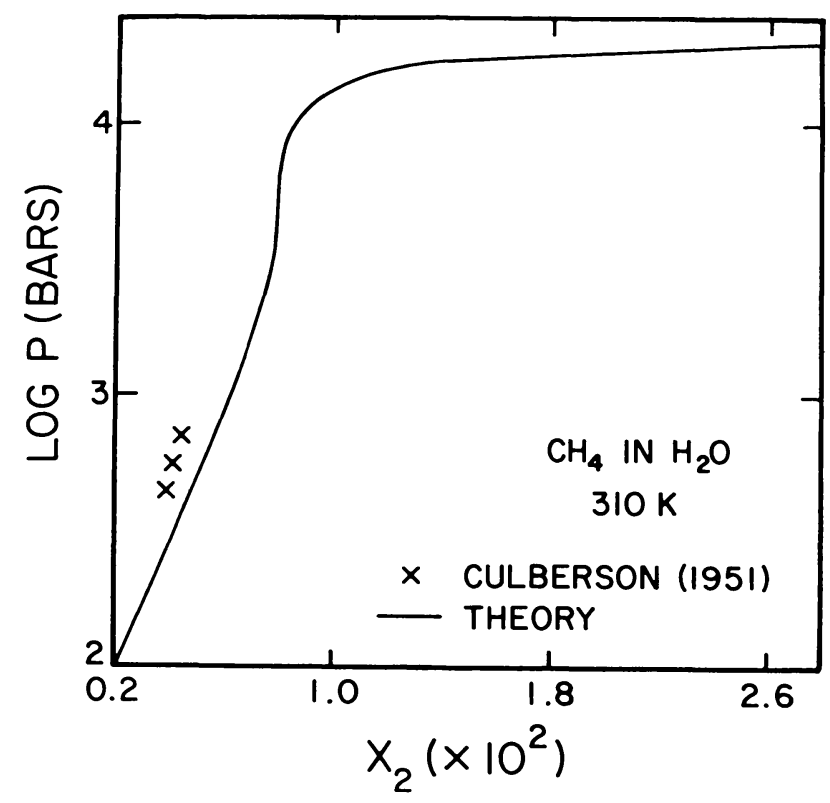

FIG. 12.- Solubility of methane in water at $310 \mathrm{~K}$ plotted as system pressure vs. methane mole fraction. 
Provided $x_{2}$ is small, the dominant correction to the chemical potential of water due to dissolved methane is $k T \ln (1-$ $\left.x_{2}\right) \approx-k T x_{2}$. With this correction applied to $\Delta \mu^{\beta}$ in equation (5), we find that at $310 \mathrm{~K}$, the dissociation pressure drops from $P=12,460$ bars at $x_{2}=0$ to 12,300 bars at $x_{2}=0.01$, and 10,590 bars at $x_{2}=0.1$. Thus, if $x_{2}<0.1$, the effect of methane solubility in a saturated solution is small.

Actually, water which is saturated (in the sense that the chemical potential of dissolved methane is equal to that of pure methane at the same $T, P$ ) is always supersaturated with respect to clathrate if the $T, P$ conditions lie within the domain of clathrate stability. We can demonstrate the magnitude of this effect as follows. Assume, for simplicity, an ideal solution containing a methane mole fraction $x_{2}$ which is less than $x_{2, \text { sat }}$, the maximum solubility as obtained above (i.e., Fig. 12). It follows that

$$
f_{2}^{\text {solution }}(T, P)=\frac{x_{2}}{x_{2, \text { sat }}(T, P)} f_{2}^{\text {pure }}(T, P),
$$

where $x_{2 \text { sat }} \ll 1$ is the saturated mole fraction of $\mathrm{CH}_{4}$ at $T, P$, and $f_{2}^{\text {pure }}$ is the fugacity of the pure methane phase at $T, P$. Using $f_{2}^{\text {pure }}$ as computed in $\S \mathrm{V} a$, we compute the region of stability of the methane clathrate as a function of degree of saturation of the water, $x_{2} / x_{2, \text { sat }} \leq 1$. This is plotted as a $P-x_{2}$ diagram at $320 \mathrm{~K}$ in Figure 13 . The stability field extends outward from the saturation value to smaller mole fractions of methane with decreasing pressure, as the difference in fugacity between pure methane and methane in clathrate reaches a maximum around 6000 bars. The limiting fraction of saturation for stability then increases again until it equals 1 at the low-pressure clathrate stability point at 2000 bars (see Fig. 10). There is thus a substantial region of intermediate pressure for which clathrate can coexist with an unsaturated water-methane solution. Another way to look at this is to note that clathrate hydrate will continue to form, at some pressures, after all the free methane is exhausted; some additional methane is drawn out of solution.

\section{c) Mixed $\mathrm{N}_{2}-\mathrm{CH}_{4}$ Clathrate}

The analysis in $\S \mathrm{V} a$ was applied to structure $\mathrm{I}_{2}$ clathrate using the extensive thermodynamic pressure-temperaturevolume data of Jacobsen and Stewart (1973) and Mel'nik (1978). The critical pressure for clathrate stability is 4-5 kilobars; extrapolating the data of Marshall, Saito, and Kobayashi (1964), the corresponding temperature is $\sim 310 \mathrm{~K}$. The shape of the phase diagram is similar to that of methane, the high-pressure limit being somewhat less than for methane, about 10 kilobars. Since the Langmuir constants for structure II $\mathrm{N}_{2}$ clathrate are predicted to be similar to or lower than those for structure I, and $\Delta V^{\beta}$ for structure II clathrate is slightly larger than for structure $\mathrm{I}$, the stability field of $\mathrm{N}_{2}$ structure II should be qualitatively similar to, but somewhat smaller than, the structure I case. Hence, even if $\mathrm{N}_{2}$ clathrate is structure II at low pressure, it will convert to structure I at some pressure below 10 kilobars. An equally strong conclusion holds for $\mathrm{CH}_{4}$ : structure I is preferred over structure II at high pressure as well as low. These conclusions are confirmed by numerical experiments but assume the compressibility $\kappa$ of

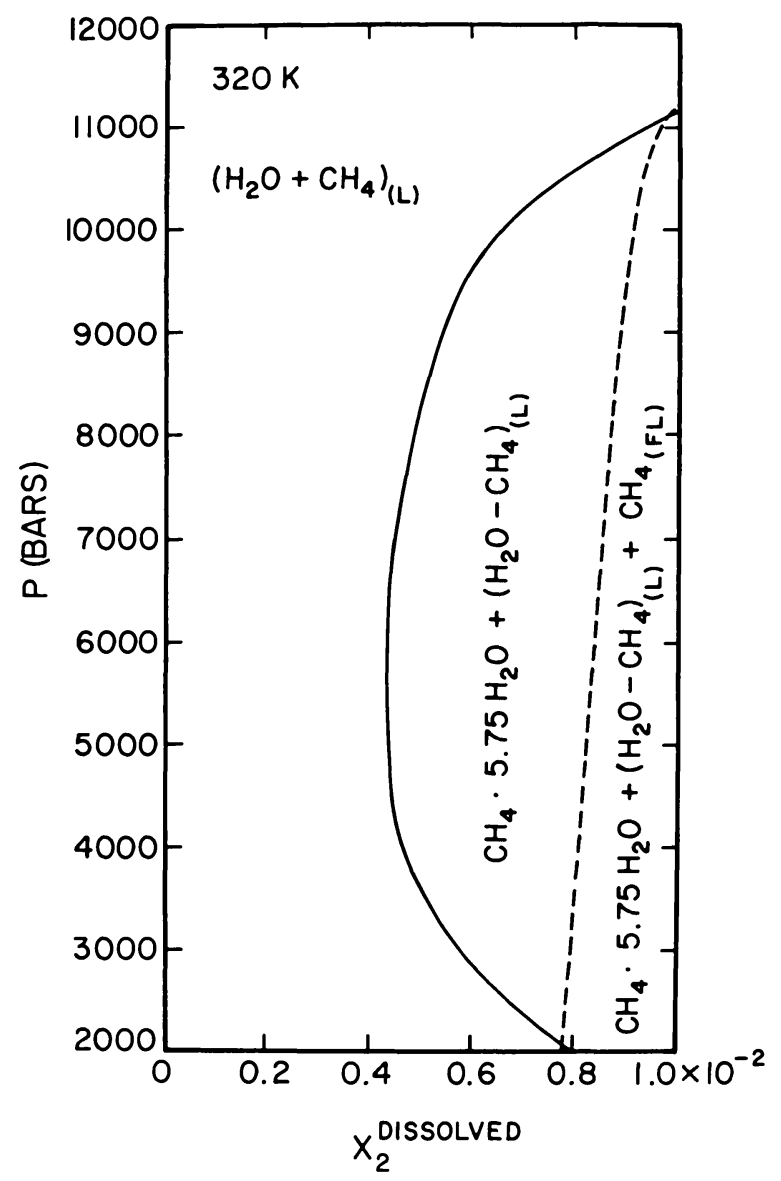

FIG. 13.-Calculated stability field of methane clathrate in coexistence with water-methane solution, plotted as system pressure vs. methane mole fraction $x_{2}$ in water (solid line). Dotted line is value of $x_{2}$ for which solution is saturated.

the two clathrate structures to be identical. Whalley (1980) concludes $\kappa_{\mathrm{II}}=1.03 \kappa_{\mathrm{I}}$. Of more interest is the variation in dissociation pressure of the structure I binary clathrate, at a given temperature, due to varying the $\mathrm{N}_{2}-\mathrm{CH}_{4}$ composition of the coexisting phase. We use equation (1) in its general form, with $j=1,2$ for methane and nitrogen. To determine the fugacities of the methane and nitrogen in the coexisting phase, we consider two alternative models. The simplest assumes $\mathrm{CH}_{4}$ and $\mathrm{N}_{2}$ form ideal solid and liquid solution; i.e.,

$$
\begin{aligned}
& f_{\mathrm{N}_{2}}^{\mathrm{N}_{2}+\mathrm{CH}_{4}}=\left(1-x_{\mathrm{CH}_{4}}\right) f_{\mathrm{N}_{2}}^{\text {pure }}, \\
& f_{\mathrm{CH}_{4}}^{\mathrm{N}_{2}+\mathrm{CH}_{4}}=x_{\mathrm{CH}_{4}} f_{\mathrm{CH}_{4}}^{\text {pure }},
\end{aligned}
$$

where the superscript $\mathrm{N}_{2}+\mathrm{CH}_{4}$ signifies the mixed phase. Low temperature and pressure studies by Omar, Dokoupil, and Schroten (1962) suggest that the solid and liquid solutions behave more as "regular" solutions:

$$
\begin{aligned}
f_{\mathrm{CH}_{4}}^{\text {reg }} & =f_{\mathrm{CH}_{4}}^{\text {ideal }} \exp \left[\frac{A}{k T}\left(1-x_{\mathrm{CH}_{4}}\right)^{2}\right], \\
f_{\mathrm{N}_{2}}^{\text {reg }} & =f_{\mathrm{N}_{2}}^{\text {ideal }} \exp \left(\frac{A}{k T} x_{\mathrm{CH}_{4}}^{2}\right),
\end{aligned}
$$


where $A$ is the energy of exchanging an $\mathrm{N}_{2}$ molecule for $\mathrm{CH}_{4}$ in solution, analogous to $A$ for $\mathrm{H}_{2} \mathrm{O}-\mathrm{CH}_{4}$ in $\S \mathrm{V} b$. Here $x_{\mathrm{CH}_{4}}$ is relative to the total $\mathrm{CH}_{4}+\mathrm{N}_{2}$ abundance in the pure phase coexisting with clathrate. Based on data in Omar, Dokoupil, and Schroten (1962) and Prausnitz (1969, p. 285), $A\left(\mathrm{~N}_{2}, \mathrm{CH}_{4}\right)$ $\approx 200 \mathrm{cal} \mathrm{mole}^{-1}$. The effect of $A$ is to increase slightly the pressure at which the binary clathrate destabilizes; at most it is a $3 \%$ effect and is not considered further.

Figure 14 plots the stability pressure of the structure I binary clathrate at $180 \mathrm{~K}$ versus the mole fraction of $\mathrm{CH}_{4}$ relative to $\mathrm{CH}_{4}+\mathrm{N}_{2}$. As expected, the clathrate composition behaves as a continuous series of "solid solutions" (not to be confused with the coexisting solid or liquid $\mathrm{N}_{2}-\mathrm{CH}_{4}$ solution). The lower curve gives the clathrate composition calculated using equation (2) for the coexisting $\mathrm{N}_{2}-\mathrm{CH}_{4}$ solution composition indicated by the upper line. Under the ambient conditions the coexisting solution is in the solid field (Grace and Kennedy 1967), although the qualitative behavior of the phase diagram is not affected if the coexisting solution is fluid. Because of the lower methane and $\mathrm{N}_{2}$ Langmuir constants in structure II clathrate and a $\Delta \mu^{\beta}$ value larger than that of structure I at high pressure, the coexisting phase is not expected to form a structure II clathrate. As noted above, this conclusion is contingent upon the compressibility of the two structures being identical. The behavior of the system may be seen by choosing an $\mathrm{N}_{2}-\mathrm{CH}_{4}$ composition in the stability field of the clathrate and increasing the pressure. Let $\left.x_{\mathrm{CH}_{4}}\right|_{\text {clathrate }}=0.2$, and increase the pressure from 10 kilobars. At point $A, P=10.3$ kilobars, a nearly pure $(92 \%) \mathrm{N}_{2}$ solution is evolved from the clathrate (with formation of water ice),

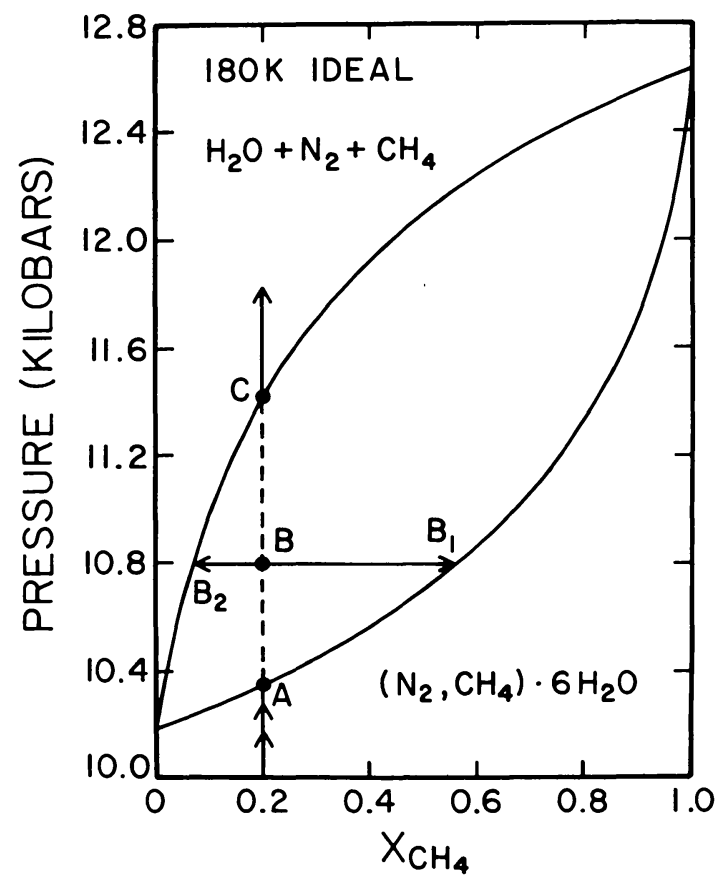

FIG. 14. - Phase diagram of binary $\mathrm{N}_{2}-\mathrm{CH}_{4}$ clathrate at $180 \mathrm{~K}$, displayed as pressure vs. $\mathrm{CH}_{4}$ mole fraction (relative to $\mathrm{N}_{2}+\mathrm{CH}_{4}$ ). The coexisting $\mathrm{N}_{2}-\mathrm{CH}_{4}$ solution is assumed to be ideal. The lower and upper curves are the composition in the clathrate and coexisting phase, respectively. Letters refer to an isothermal compression of the system discussed in text. which evolves toward more methane rich. As $P$ increases, both clathrate and coexisting solution evolve toward more methane rich, with increasing proportion of water ice + $\mathrm{N}_{2}-\mathrm{CH}_{4}$ solution to clathrate. At point $\mathrm{B}$, for example, $P=$ 10.8 kilobars, $\left.x_{\mathrm{CH}_{4}}\right|_{\text {solution }}=0.07$ and $\left.x_{\mathrm{CH}_{4}}\right|_{\text {clathrate }}=0.55$, with the fraction of $\mathrm{N}_{2}+\mathrm{CH}_{4}$ in solution versus clathrate given inversely by the ratio of chord lengths $\mathrm{B}-\mathrm{B}_{2}$ and $\mathrm{B}-\mathrm{B}_{1}$. At $\mathrm{C}$, 11.44 kilobars, all the $\mathrm{N}_{2}$ and $\mathrm{CH}_{4}$ has evolved from the clathrate, and the stable phase is now $\mathrm{N}_{2}-\mathrm{CH}_{4}$ solution plus water ice. Thus, in an icy satellite containing binary $\mathrm{N}_{2}-\mathrm{CH}_{4}$ clathrate, some zoning of coexisting volatile composition is expected, over, however, a rather narrow ( $\sim 1$ kilobar) pressure range. The same behavior is expected for the $\mathrm{CH}_{4}-\mathrm{CO}$ binary clathrate. Note also that the closer the stability pressures of the pure clathrate end members are, the more similar will be the compositions of the binary clathrate and coexisting phase.

\section{d) Clathrate Stability in the Presence of Ammonia}

We now calculate the phase diagram for high-pressure clathrate stability in the presence of water ice and ammoniawater liquid. Incorporation of $\mathrm{NH}_{3}$ in cage sites is neglected because the fugacity of $\mathrm{NH}_{3}$ in the $\mathrm{NH}_{3}-\mathrm{H}_{2} \mathrm{O}$ liquid is always low enough compared with that of the guest molecule (e.g., $\mathrm{CH}_{4}$ ) phase that $\mathrm{NH}_{3}$ occupancy relative to $\mathrm{CH}_{4}$ is $\ll 1$. As in $\S \mathrm{V} a$, we calculate $\Delta \mu^{\beta}\left(T_{0}, P\right)$ from $\Delta \mu^{\beta}\left(T_{0}, P_{0}\right)$ by integrating isothermally using equation (A3). The quantity $\Delta \mu^{\beta}\left(T_{0}, P_{0}\right)$ is found from equation (24), where $T_{f}\left(P_{0}\right)$ for the ammonia-water solution is from Haudenschild (1970). The pressure limits on the clathrate stability regime are then calculated as in $\S \mathrm{V} a$. In the water-ice field, the pressure limits must be unchanged from the pure water results. To define the boundaries of the water-ice regime, the freezing curve must be calculated over the entire pressure regime ( $0-15$ kilobars) considered. In the absence of clathratable gases,

$$
\mu_{\mathrm{H}_{2} \mathrm{O}}^{\text {ice }}=\mu_{\mathrm{H}_{2} \mathrm{O}}^{\text {solution }}=\mu_{\mathrm{H}_{2} \mathrm{O}}^{\text {liquid, pure }}+R T \ln \left(\gamma_{\mathrm{H}_{2} \mathrm{O}} x_{\mathrm{H}_{2} \mathrm{O}}\right)
$$

at the freezing curve. Here $\gamma_{\mathrm{H}_{2} \mathrm{O}}$ is the activity coefficient (Prausnitz 1969, p. 181) of water in the ammonia-water solution, a function of $x_{\mathrm{H}_{2} \mathrm{O}}$ but assumed constant with respect to temperature and pressure. Writing $d \mu=V d P-S d T$ and considering the freezing process along an isobar, we find

$$
\Delta S_{\text {freeze }}\left(T-T_{F}^{0}\right)=R T \ln \left(\gamma_{\mathrm{H}_{2} \mathrm{O}} x_{\mathrm{H}_{2} \mathrm{O}}\right)
$$

where $T_{F}^{0}$ is the freezing point of pure water at system pressure $P$, and $\Delta S_{\text {freeze }}$ is the entropy of freezing at $P$ and is tabulated for the various ice phases in Hobbs $(1974$, p. 65$)$. For a specified $x_{\mathrm{H}_{2} \mathrm{O}}$, using the melting point data of Haudenschild (1970), equation (38) is solved for $\gamma_{\mathrm{H}_{2} \mathrm{O}}$ in the low-pressure field. This value of $\gamma_{\mathrm{H}_{2} \mathrm{O}}$ is then used along with the appropriate $\Delta S_{\text {freeze }}$ to calculate the freezing curve in the high-pressure ice phases. Since $\gamma_{\mathrm{H}_{2} \mathrm{O}} \approx 0.5$ for $x_{\mathrm{H}_{2} \mathrm{O}}=0.70$, the activity coefficient clearly cannot be neglected.

The resulting ice-liquid boundary and clathrate stability region for $x_{\mathrm{H}_{2} \mathrm{O}}=0.85$ and 0.75 are plotted in Figure 15. The clathrate stability region has the same general shape as for the 


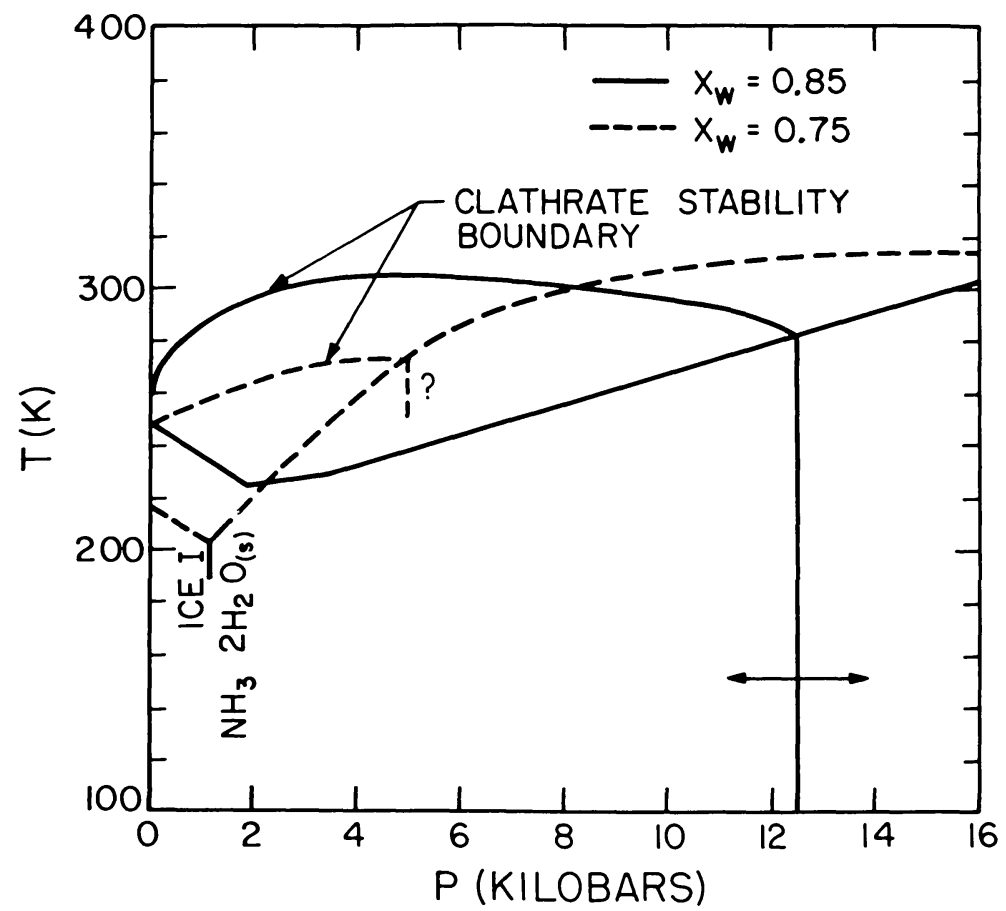

FIG. 15. - Temperature vs. pressure of $\mathrm{CH}_{4}$ clathrate stability field in presence of ammonia for $x_{\mathrm{H}_{2} \mathrm{O}}=0.85$ and 0.75 . Labeling of fields is identical with that in Fig. 10, except for the water ice-dihydrate-liquid triple-point indicated.

pure water case and is constrained to be identical in the ice field. Note that Figure 8 shows in much more detail the low-pressure end of the clathrate stability field of Figure 15; together they display the phase boundaries in the presence of ammonia at all pressures $\left(0-10^{4}\right.$ bars) of interest.

Preliminary results of Johnson, Schwake, and Nicol (1985) suggest that at $x_{\mathrm{H}_{2} \mathrm{O}}<0.85$, an ammonia-dihydrate $\left(\mathrm{NH}_{3}\right.$. $2 \mathrm{H}_{2} \mathrm{O}$ ) phase freezes from the liquid solution at high pressures. A series of temperature-composition phase diagrams at several pressures for the ammonia-water system has been calculated by the authors, using data in Johnson, Schwake, and Nicol (1985), Chan and Giauque (1964), and Rollet and Vuillard (1956). The resulting freezing boundary at $x_{\mathrm{H}_{2} \mathrm{O}}=$ 0.75 is shown in Figure 15. Because the volume of the dihydrate phase is not known, we cannot predict the clathrate stability field in the dihydrate region. However, the large $\Delta T / \Delta P$ slope of the dihydrate solidus implies a substantial volume decrease upon freezing; hence, the clathrate stability field probably terminates at a lower pressure than it would in the absence of the solidus.

\section{KINETICS OF FORMATION}

Although in the preceding sections we have been concerned with the thermodynamic aspects of clathrate formation, application to problems of nebular condensation requires consideration of the kinetics of formation. Lewis and Prinn (1980) argue, for example, that formation of clathrate at "nebular" temperatures plausible for Uranus and Neptune, i.e., $<60 \mathrm{~K}$, may be severely inhibited by the inefficiency of diffusion of large guest molecules (i.e., $\mathrm{CO}$ ) through water-ice planetesimals. The term nebula refers to disklike, primarily $\mathrm{H}_{2}$ gaseous regions out of which solar system bodies are assumed to have formed. The solar nebula is usually modeled as a low-density $\left(\sim 10^{-12} \mathrm{~g} \mathrm{~cm}^{-3}\right.$ at Saturn's orbit; Lewis 1974) disk from which the planets accreted; nebulae around giant planets from which satellites formed could have had densities $\sim 10^{-5}$ $\mathrm{g} \mathrm{cm}^{-3}$ (Prinn and Fegley 1981; Lunine and Stevenson 1982 b). We examine the issue of kinetics below and conclude that while diffusion of gas through the interior of a $10 \mathrm{~m}$-sized planetesimal of high porosity is possible over nebular time scales $\left(10^{7} \mathrm{yr}\right)$, diffusion of clathrated layers through the ice grains themselves is too slow to enclathrate most of the ice. However, collisions can expose essentially all the ice to the gas over time scales shorter than or comparable to planetesimal lifetimes in the Saturnian (and perhaps solar) nebula.

If we consider a planetesimal formed by repeated coagulation of smaller grains, we can imagine a rather porous object characterized by a hierarchy of grain and, hence, pore sizes, extending perhaps from centimeter-sized particles down to micron-sized. Each size range of particles may itself have pore systems at the next size scale down. Eventually a size $(\sim 1$ $\mu \mathrm{m})$ is reached at which porosity is not important; this is the size at which gas-phase diffusive growth acted during condensation. For an example of probable morphologies of coagulated grains, see Brownlee (1978, p. 140). One of two diffusional processes thus limits the formation of clathrate: diffusion through the porous volume of the entire planetesimal or diffusion through the smallest-scale, nonporous ice particles. 
The mean free path of a gas molecule, $k T / \sigma P$ (where $T=$ temperature, $P=$ pressure, $\sigma=$ cross section of molecule), is $\sim 2 \mathrm{~cm}$ for $\mathrm{H}_{2}$ at $T=50 \mathrm{~K}$ and $P=10^{-6}$ bars. Under such conditions, diffusion through centimeter- or smaller-diameter pores is in the so-called Knudsen regime; i.e., collisions with pore walls dominate over gas-gas collisions (Carman 1956, p. 63). The Knudsen diffusion coefficient for gas species $i$ is given in Evans, Watson, and Mason (1961):

$$
D_{i}=\frac{4}{3} K_{0}\left(\frac{8 k T}{\pi m_{i}}\right)^{1 / 2},
$$

where $m_{i}=$ mass of molecule of species $i$, and $K_{0}$ is a constant with dimensions of length, characteristic of the porous medium, and can be related to the pore diameter $d$ by a "dusty gas" model as in Evans et al.:

$$
K_{0} \approx \frac{1}{20} \frac{\varepsilon}{q} d,
$$

where $\varepsilon=$ porosity, i.e., volume fraction of pore space in the medium, and $q=$ tortuosity, which is the square of the ratio of actual distance followed by a gas streamline to the pore length $l$. The numerical factor in equation (40) was calculated from the dusty gas model assuming that the average pore size is roughly equal to the ice grain size. We take $\varepsilon>0.5$, and for many porous systems $q \leqslant 3$ (Carman 1956, p. 48), so $\varepsilon / q \sim 0.1$. Even if $d=1 \mu \mathrm{m}$, the diffusion distance is $\sim 10 \mathrm{~km}$ over $10^{7}$ yr. Hence, $\mathrm{H}_{2}$ diffuses rapidly through porous bodies.

Diffusion of other nebular gases such as $\mathrm{CO}$, noble gases, etc., is substantially slower as a result of the formation of clathrate along the ice surfaces of pores. Since, as shown later, diffusion through the solid ice is extremely slow, clathrate formation corresponds to adsorption of gas molecules on the ice.

The one-dimensional diffusion equation for a particular adsorbable gas species is (Barrer 1967)

$$
\frac{\partial c_{g}}{\partial t}=D \frac{\partial^{2} c_{g}}{\partial x^{2}}-\frac{\partial c_{a}}{\partial t}
$$

where $c_{g}$ is the gas concentration (molecules per unit volume of porous medium) in the pore space, $c_{a}$ is the concentration of molecules adsorbed on pore walls, $D$ is the Knudsen diffusion coefficient, and $t$ and $x$ are the time and space coordinates, respectively. The second term on the right-hand side represents the exchange of molecules between the adsorbed and gas phases. We have neglected the surface diffusion term in equation (41) as it appears to be less rapid than Knudsen diffusion. Following Fanale et al. (1982), we define an adsorption parameter

$$
\alpha=\left.\frac{\partial P_{a}}{\partial P}\right|_{T} .
$$

Here $P$ is the partial pressure of the gas which is diffusing through the pore, and $P_{a}$ is an effective pressure of the adsorbed or enclathrated molecules: $P_{a}=\rho_{a} K T / m$, where $\rho_{a}=$ adsorbed mass per unit volume of ice. Note that we ignore interaction between different gas species since the gas is in the Knudsen flow regime. Using the ideal gas equation to convert concentrations to pressures, and substituting equation (42) into equation (41), we find

$$
\frac{\partial P}{\partial t}=D\left(\frac{\varepsilon}{\varepsilon+\alpha}\right) \frac{\partial^{2} P}{\partial x^{2}},
$$

so that for substantial adsorption effects from clathration,

$$
D\left(\frac{\varepsilon}{\varepsilon+\alpha}\right) \sim \frac{D}{\alpha} \quad \text { for } \quad \alpha \gg 1 .
$$

Since $\alpha$ is the slope of the adsorption isotherm, we can calculate it by considering clathration as a limiting case of adsorption wherein the ratio of adsorbate to absorbent molecules is $\frac{1}{6}$ and the effective adsorption area $A_{s}$ is $\sim 5 \times 10^{6}$ $\mathrm{cm}^{2}$ per $\mathrm{cm}^{3}$ of ice (Delsemme and Miller 1970), $\sim 10-100$ times larger than for "normal" adsorption onto ice. Following Delsemme and Miller (1970), for a single gas species we employ the expression

$$
\log _{10} S_{a}=\log _{10} S_{m}-a T^{2}\left[\log _{10}(P / e)\right]^{2}
$$

to find $\alpha$. Here $S_{a}$ is the amount of gas adsorbed in molecules per $\mathrm{cm}^{2}, S_{m}$ is the monolayer coverage of ice $\left(S_{a} / S_{m}=\frac{1}{6}\right.$ at saturation), $e$ is the vapor pressure of the gas species, and $a$ is an empirical constant dependent mainly on the gas species. In their paper, Delsemme and Miller present data on methane adsorption on pyrex glass; since they claim $a$ is not strongly dependent on adsorber, we use their value. Writing $P_{a}$ in terms of $A_{s}$ and $S_{a}$ and differentiating with respect to $P$ yields

$$
\alpha=-\left(\frac{2 S_{m} A_{s} a k T^{3} \log _{10} P / e}{P}\right) \times 10^{-a T^{2}\left(\log _{10} P / e\right)^{2}} .
$$

For $T=50 \mathrm{~K}$, a methane nebular partial pressure of $8 \times 10^{-10}$ bars, $a=4 \times 10^{-6} \mathrm{~K}^{2}, S_{m}=1.5 \times 10^{14}$ molecules $\mathrm{cm}^{-2}, e=7$ $\times 10^{-6}$ bars (CRC Handbook of Chemistry and Physics, 1970, p. D-146), we find $\alpha \sim 10^{8}$, or a diffusion distance of $1 \mathrm{~m}$ over $10^{7} \mathrm{yr}$ if the pore size is $1 \mu \mathrm{m}$. A simpler calculation assuming $d P_{a} / d P=P_{a} / P$ yields $\alpha \sim 10^{9}$, In reality, planetesimal architecture probably consists of a hierarchy of pore sizes. Illustrative though exaggerated examples of this sort of architecture are the fractal geometries, discussed by Mandelbrot (1977), especially the Sierpinski sponge (which has dimension 2.7268). Avnir, Farin, and Pfeifer (1984) present experimental evidence that at the molecular scale, the surfaces of most materials exhibit fractal geometries. In most of these hierarchical structures, the diffusion time is not dominated by the smallest pores. This can be demonstrated by examination of the solutions discussed by Carslaw and Jaeger (1959) for (thermal) diffusivities which depend on some power of the spatial coordinate $x$, say. For example, the diffusivity law $D=\alpha x$, with $\alpha=$ constant, does not inhibit diffusion to or from the point $x=0$. In a corresponding fashion, we can think of $x \approx 0$ in our problem as corresponding to the position of a deep-seated narrow pore space which is accessible because of connections to progressively larger pore spaces, and is accessed in a time that depends primarily on the diffusivity for large pores. 
We conclude that clathratable gases can diffuse on the order of meters over time scales of $\leq 10^{7} \mathrm{yr}$. We now examine time scales for diffusion of clathrate gases through the ice grains themselves. That is, given that all grain surfaces are coated with a layer of clathrate, on what time scale can this layer move through the grains?

Experimental data on kinetics of clathrate formation are extremely limited. Delsemme and Wenger (1970) formed methane clathrate hydrate at $82 \mathrm{~K}$ by condensing water vapor in the presence of methane gas at appropriate pressures. Barrer and Edge (1967) formed clathrate from ice and rare gases, achieving nearly maximum theoretical incorporation of rare gases at temperatures down to $90 \mathrm{~K}$ on time scales of order $5 \mathrm{hr}$, by agitating the ice with steel balls. This procedure allowed exposure of fresh ice to continue until all the ice formed clathrate. Barrer and Ruzicka (1962) studied formation of clathrate from ice, chloroform, and rare gases in the absence of shaking. Qualitatively, their plots of gas uptake versus time at constant gas pressure show an initial steep rise over 10 minutes until some fraction of the gas was incorporated, apparently forming a surface layer on the ice grains, followed by a much flatter uptake curve, which we interpret as diffusion of the gas through the ice grains to deepen the clathrate layer. The presence of equal parts solid chloroform and water ice in the vessel resulted in structure II hydrate formation, in which small cavities are occupied by xenon and the larger by the chloroform according to the formula $\mathrm{CHCl}_{3} \cdot 2 \mathrm{Xe} \cdot 17 \mathrm{H}_{2} \mathrm{O}$. This, along with the imprecision of reading small changes in gas uptake from the curve, makes our diffusion coefficient derived from the data at best an order-ofmagnitude estimate for the structure I hydrate.

To derive the diffusion coefficient, we require an expression giving gas uptake as a function of time. Assume the ice grains are spherical. Assuming $D$ is constant,

$$
\frac{\partial C}{\partial t}=D\left(\frac{\partial^{2} C}{d r^{2}}+\frac{2}{r} \frac{\partial C}{\partial r}\right)
$$

where $r$ is the radial coordinate measured from the center of the sphere, $C=$ gas concentration. Making the substitution $u=C r$ yields

$$
\frac{\partial u}{\partial t}=D \frac{\partial^{2} u}{\partial r^{2}}
$$

We specify the initial boundary conditions for a sphere of radius $a$ :

$$
\begin{array}{lll}
u_{1}=0 & \text { at } r=0 & \text { for all } t, \\
u_{2}=a C_{2} & \text { at } r=a, & \\
u=r C_{0} & \text { at } t=0 & \text { for } 0<r<a ;
\end{array}
$$

which has the solution (Barrer 1941, p. 29)

$$
C=C_{2}+\frac{2 a}{\pi r} \sum_{1}^{\infty} \frac{(-1)^{n}}{n} \sin \frac{n \pi r}{a} \exp \left(-\frac{D n^{2} \pi^{2} t}{a^{2}}\right)\left(C_{2}-C_{0}\right) .
$$

When $C_{2}>C_{0}$, absorption takes place. Since the measured quantity is the total amount of gas $Q$ taken up by the clathrate at time $t$, we write

$$
\begin{aligned}
Q & =-\int_{0}^{t} D\left(\frac{\partial C}{\partial r}\right)_{r=a} d t \\
& =\frac{\left(C_{2}-C_{0}\right)_{a}}{4}\left[1-\frac{8}{\pi^{2}} \sum_{1}^{\infty} \frac{1}{n^{2}} \exp \left(-\frac{D n^{2} \pi^{2} t}{a^{2}}\right)\right] .
\end{aligned}
$$

The ratio of gas taken up at time $t$ to that at $t=\infty \equiv Q_{\infty}$ (maximum which can be incorporated) is

$$
\frac{Q}{Q_{\infty}}=1-\frac{8}{\pi^{2}} \sum_{n=1}^{\infty} \frac{1}{n^{2}} \exp \left(-\frac{D n^{2} \pi^{2} t}{a^{2}}\right),
$$

which is given in Carslaw and Jaeger (1959, p. 234) in terms of error functions as

$$
\frac{Q}{Q_{\infty}}=\frac{8}{a}\left(\frac{D t}{\pi}\right)^{1 / 2}-\frac{4 D t}{a^{2}}+\frac{16(D t)^{1 / 2}}{a} \sum_{n=1}^{\infty} \operatorname{ierfc} \frac{n a}{(D t)^{1 / 2}}
$$

For times small compared with those required for substantial uptake we approximate equation (52) by the first term and solve for $D$ :

$$
D=\frac{\pi}{64}\left(\frac{Q}{Q_{\infty}}\right)^{2} \frac{a^{2}}{t}
$$

Recalling that $a$ is the effective radius of the ice spheres, we use the data of Barrer and Edge (1967) and Barrer and Ruzicka (1962) to compute $a$ from the initial portion of the uptake curve. We find $a \sim 10^{-5}$ to $10^{-4} \mathrm{~cm}$ and hence $D \sim$ $10^{-18} \mathrm{~cm}^{2} \mathrm{~s}^{-1}$ at $200 \mathrm{~K}$. Thus, at $200 \mathrm{~K}$ it is possible for clathration to occur throughout micron-sized particles over time scales $\sim 10^{3} \mathrm{yr}$. However, since the diffusion process which moves material into the grains involves an activation energy, so that

$$
D=D_{T=200 \mathrm{~K}} \exp \left[-\frac{E}{k}\left(\frac{1}{T}-\frac{1}{200}\right)\right],
$$

then at $50 \mathrm{~K}$ diffusion will be greatly inhibited. If we use an activation energy appropriate to diffusion of hydrogen fluoride through ice, $\sim 4.5 \mathrm{kcal} \mathrm{mole}^{-1}$ (Haltenhorth and Klinger 1969) (a severe approximation since the hydrogen fluoride and clathrate guest molecule diffusion may proceed by different mechanisms), we find $D \sim 10^{-23} \mathrm{~cm}^{2} \mathrm{~s}^{-1}$ at $100 \mathrm{~K}$ and $\sim 10^{-33} \mathrm{~cm}^{2} \mathrm{~s}^{-1}$ at $50 \mathrm{~K}$ ! It is apparent that if the Barrer and Ruzicka data have been correctly interpreted, diffusion of gas into solid grains to form clathrate is negligible at temperatures of interest.

It is important to realize, however, that even in the absence of diffusion and shaking, about $1 \%$ of the maximum possible amount of xenon gas is incorporated into the ice particles as a monolayer of clathrate, in the experiments of Barrer and 
Ruzicka. If the ice grains in their experiments are at all representative of those in a porous planetesimal structure, then one may conclude that at least $\sim 1 \%$ of the available $\mathrm{CO}$ or $\mathrm{CH}_{4}$ (whatever is the predominant carbon species) will be incorporated as clathrate. Thus, we claim that carbon incorporated in clathrate will be at least of the same order of abundance as $\mathrm{CO}_{2}$ condensate, the latter comprising $\lesssim 1 \%$ of the available carbon from the nebular gas (Lewis and Prinn 1980).

It is possible that enclathrated $\mathrm{CH}_{4}$ or $\mathrm{CO}$ may still be the dominant source of condensed carbon. Experiments by Barrer and Ruzicka (1962), Barrer and Edge (1967), and others indicate essentially complete conversion of the ice to clathrate when the ice is agitated and broken up, suggesting that the clathrate particles are much more likely to be dislodged, exposing fresh ice, than they are to be destroyed by the agitation.

An analogous process in a primordial gaseous nebula is planetesimal collision. A porous planetesimal in contact with the gas would consist of ice grains coated with clathrate; upon collision some of the coating would be stripped off, exposing fresh ice. Since laboratory work suggests that the bonding of the clathrate layer to the underlying ice is weaker than the water bonding within the ice, this process is expected to predominate over fracturing of ice itself. Below we develop a crude model to test whether a planetesimal can be eroded completely (i.e., all ice exposed to gas) in a time less than the accretion time, gas drag infall time, etc., of the planetesimals, or the lifetime of the nebula itself.

Consider meter-sized (or larger), porous particles (hereafter referred to as planetesimals although the term is normally reserved in the literature for later, larger objects) composed of micron-sized grains. This is one possible stage for proto-satellite or planet material. We can find the maximum mass of material spalled off of grains during collision as a function of velocity, by equating all of the kinetic energy of colliding planetesimals to the surface bonding energy of the fragment, so that $\Delta m / m=\rho \Delta r v^{2} / \sigma$, where $\Delta m$ and $m$ are fragment and grain mass, $\sigma$ the bonding energy per unit area (probably 0.1-0.01 that of the ice hydrogen bond), $\rho$ the grain density, $\Delta r$ the fragment thickness (taken to be the thickness of the clathrate layer), and $v$ the collisional velocity. We choose $\rho \sim 1 \mathrm{~g} \mathrm{~cm}^{-3}, \Delta r \sim 10^{-7} \mathrm{~cm}$, and $\sigma=10 \mathrm{ergs} \mathrm{cm}^{-3}$, so $\Delta \mathrm{m} / \mathrm{m}$ $=10^{-8} v^{2}$, with $v$ in $\mathrm{cm} \mathrm{s}^{-1}$.

We assume $v$ to be due to velocity differences among planetesimals moving through a gaseous disk. The most plausible source of such differences is gas drag acting on particles of different radii. From Weidenschilling (1977), we can estimate the radial planetesimal velocity due to gas drag for two drag regimes, that of Saturnian (continuum drag, nebular density $\rho_{G}=10^{-5} \mathrm{~g} \mathrm{~cm}^{-3}$ ) and solar nebulae (Epstein drag, $\left.\rho_{G} \approx 10^{-12} \mathrm{~g} \mathrm{~cm}^{-3}\right)$. We find $v_{R}=100 r_{p}^{1 / 2} \rho_{p}^{1 / 2}$, and $v_{R}=$ $1000 r_{p} \rho_{p}$, respectively, where $r_{p}$ and $\rho_{p}$ are the planetesimal radius and bulk density, in cgs units. The dependence on $r_{p}$ implies that the velocity dispersion $v$ is $\sim v_{r} \sim 100-1000$ $\mathrm{cm} \mathrm{s}^{-1}$ for meter-sized objects. To be conservative, we adopt $v \sim 10-100 \mathrm{~cm} \mathrm{~s}^{-1}$; hence, $\Delta \mathrm{m} / \mathrm{m} \sim 10^{-6}$ to $10^{-4}$, or at least $10^{4}-10^{6}$ collisions are required to expose all the ice to clathration.
We calculate the time required for a planetesimal to suffer $m / \Delta m$ collisions as $t=(m / \Delta m) / F$, where the collision frequency $F$ is the ratio of the radial velocity of the planetesimals to their mean free path. The latter is calculated by taking the mass $M$ required to produce a particular planet or satellite and spreading it out as meter-sized planetesimals over a disk of semimajor axis $a$ and vertical thickness $v_{z} / \Omega$, where $\Omega$ is the Keplerian angular velocity and $v_{z}$ is the vertical velocity dispersion, assumed to be $\sim v_{R}$. Then

$$
t \sim \frac{m}{\Delta m}\left(\frac{\pi \rho_{p} r_{p} a^{2}}{\Omega M}\right),
$$

where the density of a planetesimal $\rho_{p}$ is $\sim 10^{-1}$ to $10^{-2}$ for high porosity.

For proto-Titan planetesimals orbiting Saturn, $t \approx 10 \mathrm{yr}$; for proto-Saturn planetesimals in the solar nebula, $t \approx 10^{6} \mathrm{yr}$. Note that for the Saturnian nebula case, $t$ is independent of $r_{p}, \rho_{p}$; for the solar nebula, $t \propto\left(r_{p} \rho_{p}\right)^{-1}$; hence, larger or denser planetesimals either do not change $t$ or make it smaller. The former value is short enough that complete clathration of planetesimals is possible prior to accretion as satellites or infall into Saturn (see, e.g., Lunine and Stevenson 1982b); the latter time scale is marginally short enough that clathration of Saturn-forming planetesimals could have occurred. Since $t \propto$ $a^{4} \rho_{G}^{2}$ for the solar nebula case, out at Neptune only a small fraction of planetesimal material may have clathrated, even over the lifetime of the solar nebula itself.

We also examined the possibility that sublimation and recondensation of water ice in the nebula constitute an important mechanism for clathrate formation, since $\mathrm{H}_{2} \mathrm{O}$ condensation in the presence of sufficient guest molecule gas pressure produces clathrate, as shown by the experiments of Delsemme and Wenger (1970). We write the rate of evaporation $f_{s}$ over a surface into a vacuum as

$$
f_{s}=\frac{P_{v}(T)}{\sqrt{ }(2 \pi m k T)},
$$

where $P_{v}=$ saturation vapor pressure of water ice (values from Eisenberg and Kauzmann 1969, p. 60), $T$ is temperature, and $m$ is the mass of a water molecule. Assuming the ice to be spheres, we calculate the time to evaporate such spheres as a function of radius and temperature and display the result in Figure 16. It is clear that the very low vapor pressure of water ice at the relevant temperatures results in excessively long time scales (i.e., $>10^{6} \mathrm{yr}$ ) for evaporative formation of clathrate, even from micron-sized grains. This mechanism may be important, however, in forming clathrate after planetesimal collisions, which produce localized heating and enhanced sublimation in the colliding planetesimals (in addition to disturbing interior ice grains and exposing fresh ice to the gas).

We suggest in conclusion that under plausible conditions in Saturnian and solar nebulae, it is possible that all of the ice could be converted to clathrate. An exception may be the outermost portions of the solar nebula (i.e., the Neptune formation zone) where collisions may have been too infrequent to expose the ice over the lifetime of the nebula. 


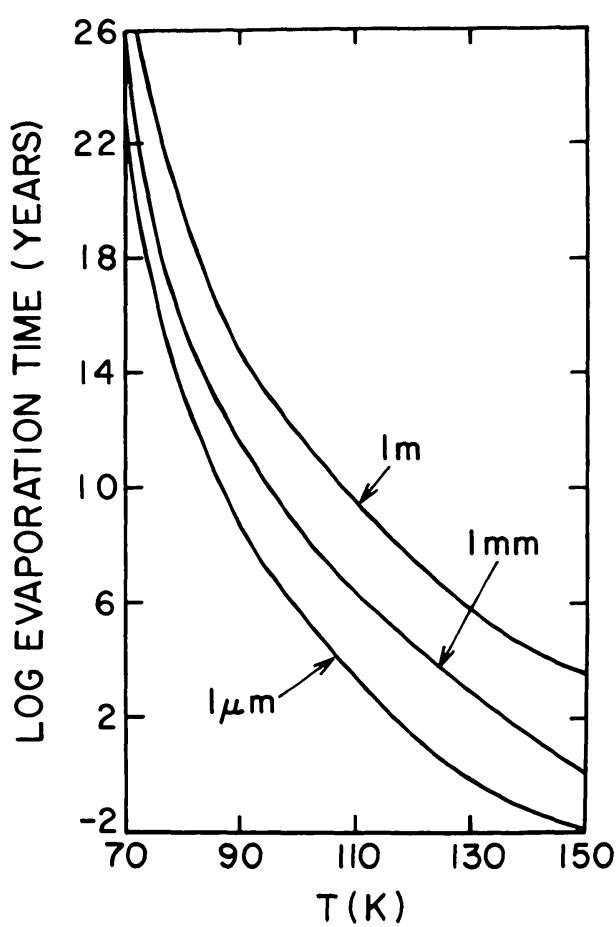

FIG. 16. - Time to evaporate a water-ice sphere in vacuum vs. temperature. Numbers on plot refer to radius of sphere.

\section{APPLICATIONS}

\section{a) Application to Primordial Nebulae}

Having compiled, in $\S$ III, a set of dissociation pressures for clathrate containing single types of guest molecules, we now calculate the relative abundances of guests incorporated in clathrate from a coexisting gas of specified composition. We will see that the abundance pattern in the clathrate is strikingly different from that in the coexisting gas and can lead to distinctive signatures of certain molecules accreted as clathrate into satellites and giant-planet atmospheres.

Consider a gas composed of molecules $j=1,2, \ldots$, each with partial pressure $P_{j}$. Equation (2) gives the fraction of cages $y_{j}$ occupied by species $j$. We can then determine the relative abundances of species $k$ and $\ell$ in each type of cage, and sum these to get the abundance ratio of species $k$ and $\ell$ in clathrate:

$$
\begin{aligned}
f_{k \ell}= & \frac{y_{1 k}+3 y_{2 k}}{y_{1 \ell}+3 y_{2 \ell}} \\
= & {\left[a_{1} \frac{C_{1 k} P_{k}\left(1+C_{2 k} P_{k}+C_{2 \ell} P_{\ell}\right)}{1+C_{1 k} P_{k}+C_{1 \ell} P_{\ell}}+a_{2} C_{2 k} P_{k}\right] / } \\
& {\left[a_{1} \frac{C_{1 \ell} P_{\ell}\left(1+C_{2 k} P_{k}+C_{2 \ell} P_{\ell}\right)}{1+C_{1 k} P_{k}+C_{1 \ell} P_{\ell}}+a_{2} C_{2 \ell} P_{\ell}\right] }
\end{aligned}
$$

where $P_{k}, P_{\ell}$ are the partial pressure of $k$ and $\ell$ in the coexisting gas, $a_{1}$ and $a_{2}$ are 1 and 3 , and 2 and 1 , respectively, for structure I and II clathrate, and Langmuir constants are calculated for the structural type (I or II) preferred by the major clathrate-forming gas. If the large- and small-cage Langmuir coefficients are not too different (both for gases $k$ and $\ell$ ), then the following expression holds:

$$
f_{k \ell}=\frac{P_{k}}{P_{\ell}} \frac{P_{\ell}^{0}}{P_{k}^{0}}
$$

where $P_{j}^{0}$ is the dissociation pressure of clathrate of given structural type in coexistence with pure gas $j$. For the gases that we consider, equation (57) does not strictly hold, and $P$ can differ by as much as a factor of $\sim 10$ when computed using equation (57) instead of equation (56).

We apply equation (56) to models of gaseous nebulae surrounding the Sun and giant planets. Abundances of elements are from Anders and Ebihara (1982). Relative abundances of molecules containing these elements (particularly C, $\mathrm{O}$, and N) are controversial; Lewis and Prinn (1980) and Prinn and Fegley (1981) argue that $\mathrm{CO}$ and $\mathrm{N}_{2}$ are the dominant carbon and nitrogen species in the solar nebula, while $\mathrm{CH}_{4}$ and $\mathrm{NH}_{3}$ dominate in a high-pressure protosatellite nebula. An important assumption implicit in the work of Lewis and Prinn (1980) is that all of the $C, N$, and $O$ is present in the gas phase or able to participate in gas-phase chemistry. Greenberg (1983) and associates suggest that a large fraction of those atoms could be locked up in grains as polymeric compounds, which in the outermost solar nebula may never be heated sufficiently to return to the gas phase. Such material could comprise comets. Even if most of the C, $\mathrm{N}$, and $\mathrm{O}$ is in the gas phase, it is possible that $\mathrm{CH}_{4} / \mathrm{CO}$ and $\mathrm{N}_{2} / \mathrm{NH}_{3}$ ratios in the outer solar system may reflect interstellar abundances rather than nebular processes. We assume that $\mathrm{N}_{2}$ is present in both solar and giant-planet nebulae as it is of interest to determine its maximum degree of incorporation in clathrate for satellite evolution models. Recall that $\mathrm{NH}_{3}$ does not incorporate primarily in clathrate but binds chemically to water ice. At low temperatures $(T<170 \mathrm{~K})$ the only effect of $\mathrm{NH}_{3}$ is to remove $\leq 15 \%$ of the water ice available for clathrate formation. In what follows, we assume all components are in good thermodynamic contact; the issue of kinetics of clathrate formation in a low-temperature/pressure environment was discussed above.

Table 3 lists the assumed gas-phase abundances. Figure 17

\begin{tabular}{|c|c|c|}
\hline Molecule & $X_{\mathrm{CH}_{4}}$ & $X_{\mathrm{CO}}$ \\
\hline $\mathrm{H}_{2} \mathrm{O}$ & $1.48 \times 10^{-3}$ & $5.90 \times 10^{-4}$ \\
\hline $\mathrm{CH}_{4}$ & $8.90 \times 10^{-4}$ & 0 \\
\hline $\mathrm{CO}$. & 0 & $8.90 \times 10^{-4}$ \\
\hline $\mathrm{N}_{2}$... & $9.10 \times 10^{-5}$ & $9.10 \times 10^{-5}$ \\
\hline $\mathrm{Ne} \ldots \ldots \ldots \ldots \ldots \ldots$ & $2.83 \times 10^{-4}$ & $2.83 \times 10^{-4}$ \\
\hline $\operatorname{Ar} \ldots \ldots \ldots \ldots$ & $7.65 \times 10^{-6}$ & $7.65 \times 10^{-6}$ \\
\hline $\mathrm{Kr} \ldots \ldots \ldots \ldots \ldots$ & $3.33 \times 10^{-9}$ & $3.33 \times 10^{-9}$ \\
\hline $\mathrm{Xe} \ldots \ldots \ldots \ldots \ldots$ & $3.20 \times 10^{-10}$ & $3.20 \times 10^{-10}$ \\
\hline
\end{tabular}
plots dissociation pressure versus temperature for major struc-

TABLE 3

Gas abundances (Mole Fraction) Relative to $\mathrm{H}_{2}$ in Nebula

NoTE.-Abundances computed from Anders and Ebihara 1982. $X_{\mathrm{CH}_{4}}$ and $X_{\mathrm{CO}}$ refer to nebulae where all carbon is in the form of $\mathrm{CH}_{4}$ or $\mathrm{CO}$, respectively. All nitrogen is assumed to be in the form of $\mathrm{N}_{2}$. Only the top three entries differ for the two types of nebulae. 


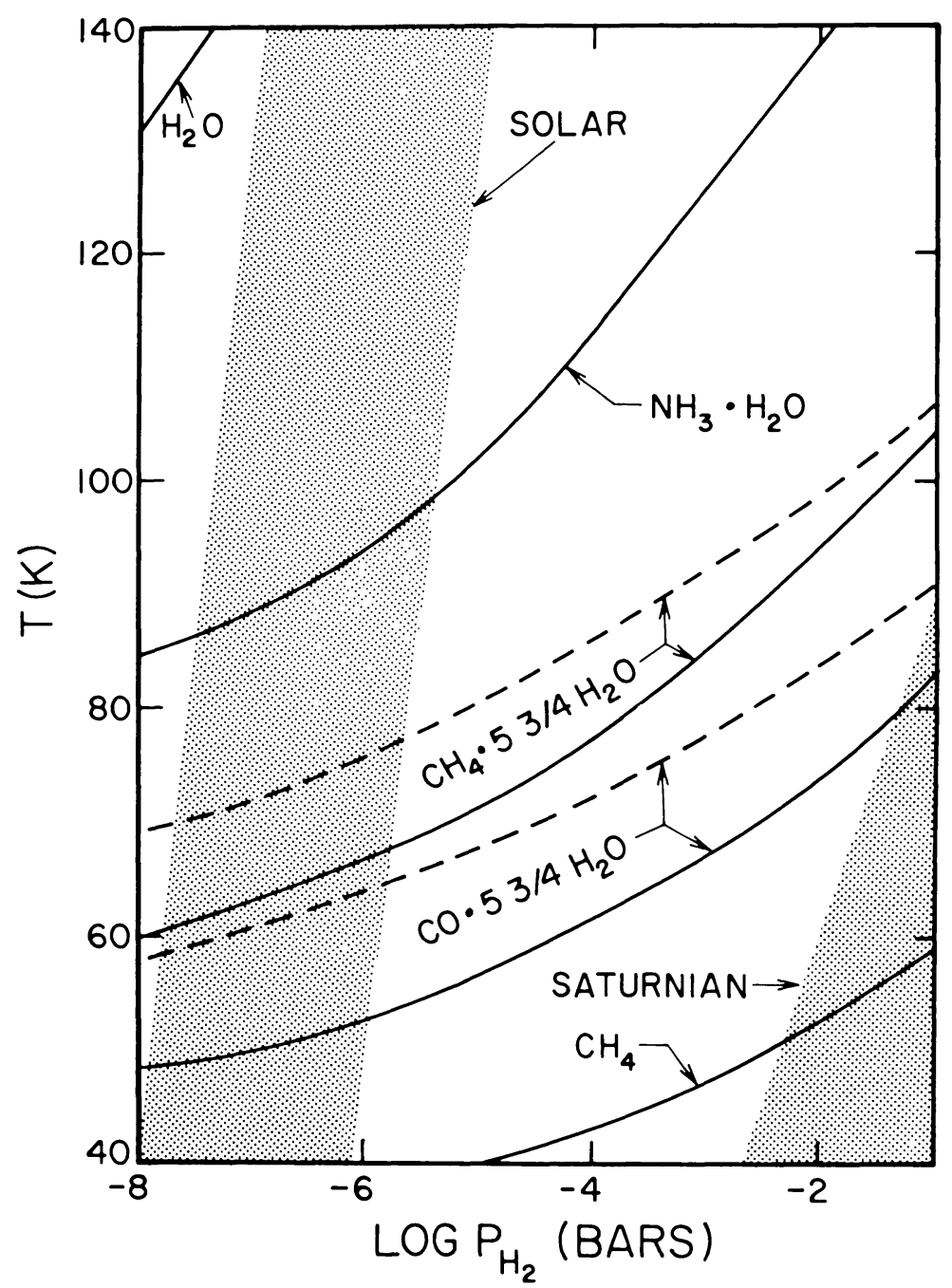

FIG. 17.-Condensation temperatures of selected major volatiles in a solar composition gaseous nebula, plotted vs. $\mathrm{H}_{2}$ pressure. The $\mathrm{CH}_{4}$ and $\mathrm{CO}$ clathrate lines apply separately to cases in which each comprises the full solar carbon abundance. Solid and dashed lines for the clathrates refer to formation from hexagonal and amorphous ice, respectively. Shaded areas are plausible pressure-temperature fields for solar and Saturnian nebular models as discussed in text.

ture I clathrate formers in the absence of other gases. Results for structure II clathrate are similar. "Major" is here defined as a gas which is capable of tying up most of the available water as clathrate. Plausible $P-T$ regions for solar and protosatellite nebulae are also shown, from Lewis (1974), Prinn and Fegley (1981), Lunine and Stevenson (1982b), and Pollack and Consolmagno (1984). The plot indicates that in a nebula around Saturn, for $T \leq 100 \mathrm{~K}$ and $P \leq 0.1$ bars, most of the available water ice is in the form of clathrate; for the solar nebula, $T \sim 50-60 \mathrm{~K}, P \sim 10^{-6}$ to $10^{-5}$ bars. The dashed lines show formation regions for $\mathrm{CH}_{4}$ and $\mathrm{CO}$ clathrates in the presence of amorphous water ice. The clathrate boundaries are increased in temperature for a given pressure. The metastability of amorphous ice implies, however, that it does not form upon cooling of ice I. Its importance under nebular conditions is therefore uncertain, and we choose $T=60$ and $100 \mathrm{~K}$, respectively, to evaluate abundance patterns in clathrate in solar and giant-planet nebulae.
Using Table 3, equation (56), and the Langmuir constants derived in $\S$ III, we compute the ratio of various gases incorporated in clathrate. Some species incorporate so highly in the clathrate that their presence in the gas is fully depleted; their abundance is then the total initial abundance in the gas. Figures $18 a$ and $18 b$ depict $f$ for the $\mathrm{CH}_{4}$-rich and CO-rich cases, respectively. Figures $19 a$ and $19 b$ display the abundance of molecules in clathrate relative to initial abundance in the nebula; that is, gases which incorporate completely into clathrate display a value of $\log (1)=0$ in this figure. Both CO and $\mathrm{CH}_{4}$ clathrates are assumed to be structure I. If $\mathrm{CO}$ or $\mathrm{CH}_{4}$ clathrate is structure II, $\mathrm{Ar}, \mathrm{Ne}$, and $\mathrm{H}_{2}$ abundances are increased by a factor of 5-10 and $\mathrm{N}_{2}$ by $\sim 2$; these increases do not affect our conclusion. In the $\mathrm{CH}_{4}$ case, $\mathrm{Kr}$ is increased by $\sim 5$ if structure II is stable; this affects our calculation of abundances in Jovian atmospheres contaminated by clathrate as indicated below. Ratios may be taken of the various molecules to determine the altered abundance pattern in clathrate. 


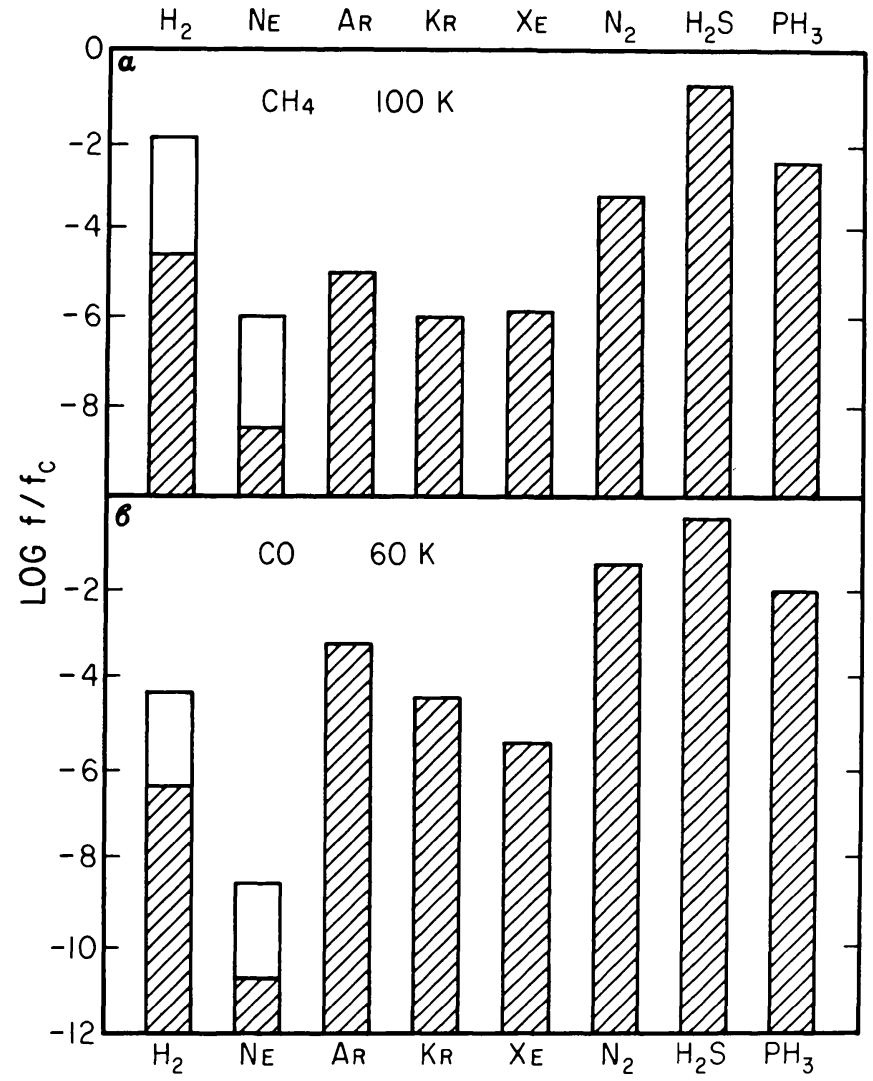

FIG. 18. - Abundance of volatiles from a solar composition gas incorporated in structure I clathrate dominated by $(a) \mathrm{CH}_{4}$ and $(b) \mathrm{CO}$ relative to the amount of $\mathrm{CH}_{4}$ or $\mathrm{CO}$ incorporated. All sulfur is assumed to be in $\mathrm{H}_{2} \mathrm{~S}$, all $\mathrm{P}$ in $\mathrm{PH}_{3}$, and all $\mathrm{N}$ in $\mathrm{N}_{2}$. Unshaded bars refer to maximum double occupancy by $\mathrm{H}_{2}$ and $\mathrm{Ne}$.

Of particular interest are (1) the noble gas ratios and (2) the ratios of $\mathrm{N}_{2}, \mathrm{CO}$, and $\mathrm{CH}_{4}$ in the clathrate.

$\mathrm{CO}_{2}$ is not shown, because of large uncertainties in its gas ahundance relative to $\mathrm{CO}$ and $\mathrm{CH}_{4}$. Note also the existence of a "lower critical decomposition pressure" for $\mathrm{CO}_{2}$ at $\sim 120 \mathrm{~K}$ (Miller and Smythe 1970). Below this temperature the vapor pressure of $\mathrm{CO}_{2}$ condensate is less than the dissociation pressure of pure $\mathrm{CO}_{2}$ clathrate, and hence the latter is not stable relative to solid $\mathrm{CO}_{2}$. However, some $\mathrm{CO}_{2}$ will always be incorporated in the mixed clathrate in the nebula, given the high propensity for $\mathrm{CO}_{2}$ to do so. We thus expect almost all available $\mathrm{CO}_{2}$ to be present in condensed material either as pure condensate or in clathrate, the relative proportion of the two being dependent on ambient nebular conditions and $\mathrm{CO}_{2}$ abundance.

Figure 20 shows the $\mathrm{Ne}, \mathrm{Kr}$, and $\mathrm{Xe}$ abundances relative to $\mathrm{Ar}$ in the structure I clathrate relative to these ratios in a solar composition $\mathrm{CH}_{4}$-rich gas, as a function of temperature. The striking enhancement of $\mathrm{Xe}$ and $\mathrm{Kr}$, and depletion of $\mathrm{Ne}$, relative to $\mathrm{Ar}$ is clearly evident. These abundance patterns are similar to, but generally more pronounced than, those seen in meteorites and terrestrial-planet atmospheres (Fig. 21) (Mukhin 1983; Donahue and Pollack 1983). The meteoritical enhancements are likely caused by physical adsorption, which, because it involves the same sort of guest-host interaction as clathration (Delsemme and Miller 1970), would be expected to

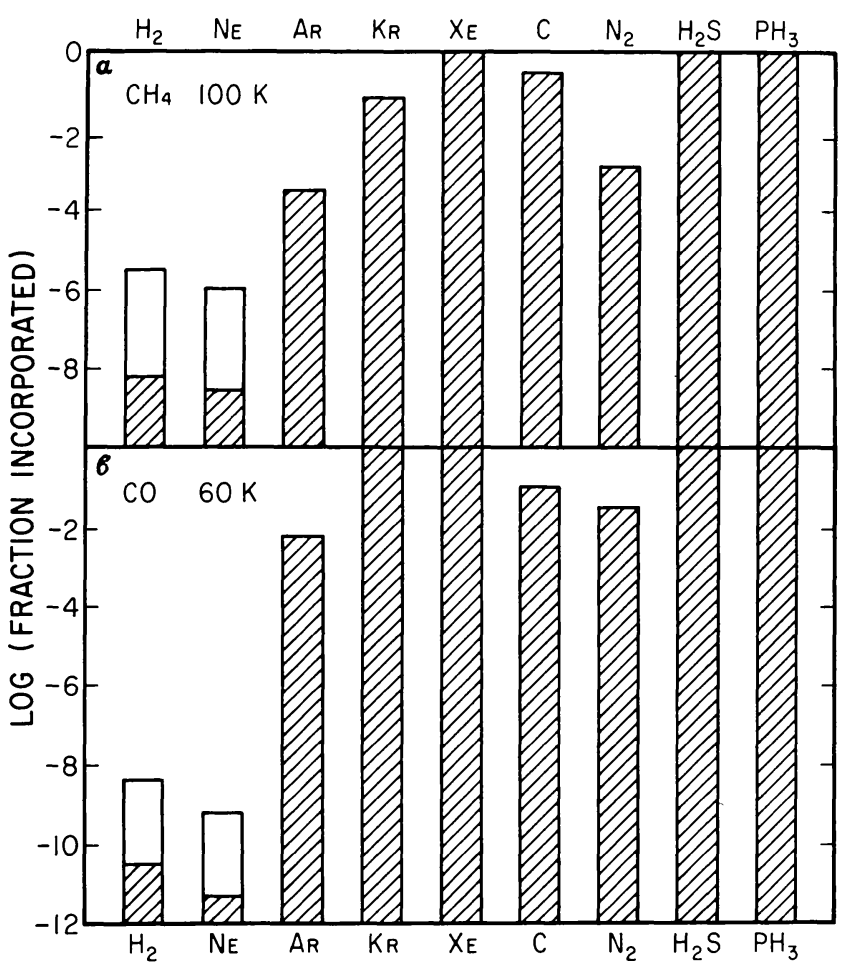

FIG. 19. - Fraction of volatiles incorporated in clathrate relative to total available in solar composition gas, for $(a) \mathrm{CH}_{4}$-dominated and (b) $\mathrm{CO}$-dominated clathrate. A log value of zero means essentially all of that species is sequestered in clathrate. "C" refers to $\mathrm{CO}$ or $\mathrm{CH}_{4}$ as appropriate. Unshaded bars assume double occupancy by $\mathrm{H}_{2}$ and $\mathrm{Ne}$.

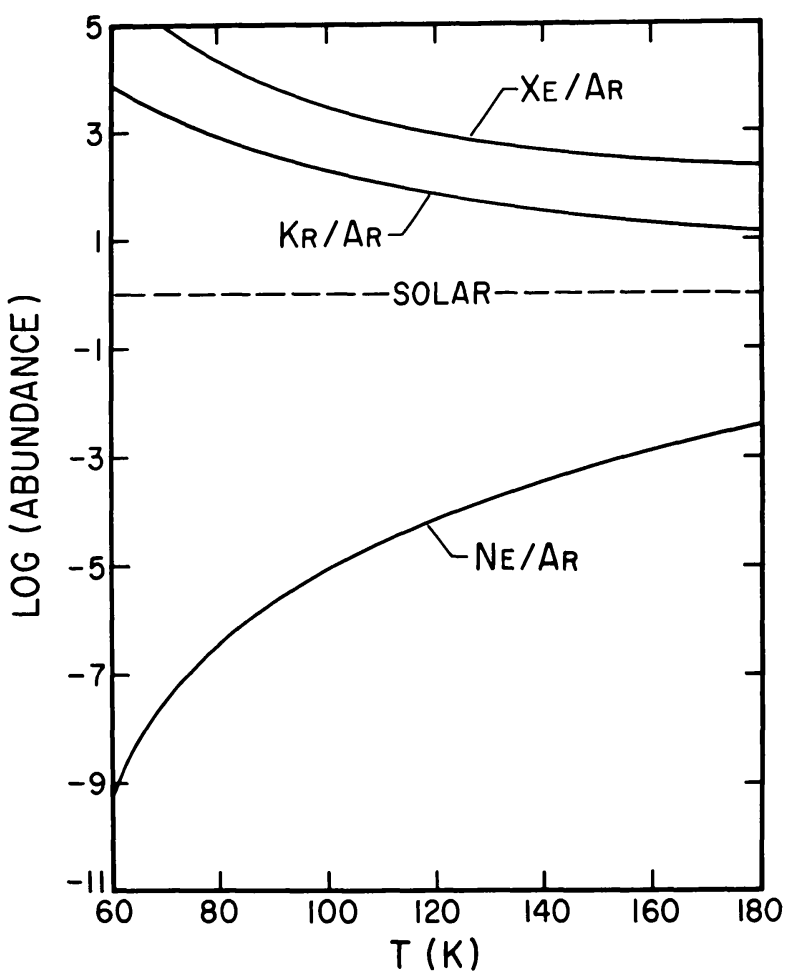

FIG. 20.- Noble gas abundance ratios in $\mathrm{CH}_{4}$-dominated clathrate relative to abundance ratios in solar composition gas, as a function of temperature. Double occupancy by $\mathrm{Ne}$ is neglected. 


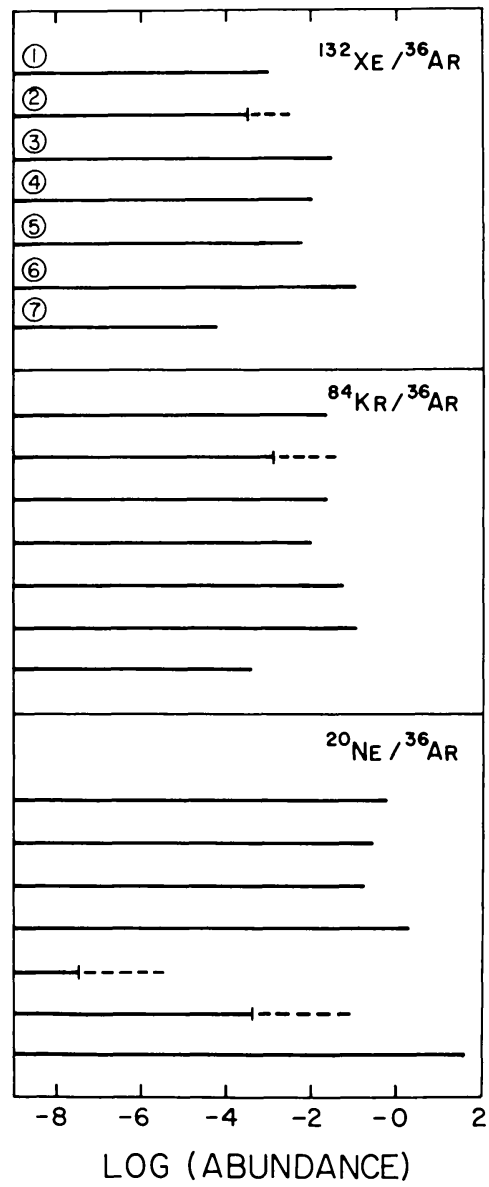

FIG. 21.-Abundance (number) ratios of noble gases in clathrate compared with solar abundance, meteorites, and terrestrial-planet atmospheres. Key: (1) Earth atmosphere, (2) Venus atmosphere, (3) carbonaceous chondrites, (4) normal chondrites, (5) CO-dominated clathrate, (6) $\mathrm{CH}_{4}$-dominated clathrate, (7) solar. (1)-(4) from Mukhin (1983), Donahue and Pollack (1983), and Pollack and Black (1982). Dashed extensions of $\mathrm{Ne}$ abundance in (5) and (6) assume double occupancy.

produce similar partitioning. The maximum uptake possible in surface adsorption processes is, however, much less than in clathration, so in low-temperature conditions where water ice is in good thermodynamic contact with surrounding gas, the incorporation and partitioning of molecules in clathrate dominate.

The noble gas enhancement may have observable consequences. It has been suggested (Stevenson 1982 b) that the observed twofold enhancement of carbon relative to solar abundance in the atmosphere of Jupiter (Gautier et al. 1982) could be due to the accretion of several Earth masses of cometary material onto the planet. This material could contain carbon as $\mathrm{CH}_{4}$ or $\mathrm{CO}$ ices or as these gases enclathrated in water ice. Temperatures and pressures in the environment of the accreting Jupiter (Lewis 1974) were probably not conducive to the formation of methane clathrate or solid methane; hence, the initial supply of methane in the Jovian envelope would have been gaseous and presumably solar in abundance. Doubling the gaseous methane abundance implies dredging more than 2 Earth masses of $\mathrm{CH}_{4}$ from the core, an unrea- sonable quantity if the core material did not originally contain $\mathrm{CO}$ or $\mathrm{CH}_{4}$ clathrate. Alternatively, if the enhanced carbon were derived from clathrate-bearing planetesimal debris, the noble gas signature would be distinctive. Figure 22 plots the noble gas abundance ratios as a function of total carbon enhancement in the Jovian atmosphere; the enhancement is assumed due to clathrate dominated by $\mathrm{CO}$ (top) or $\mathrm{CH}_{4}$ (bottom) impacting the initially solar composition atmosphere. Measurement of noble gas abundances along with methane abundance by the Galileo probe could thus test the above hypothesis against others which do not involve clathrate as the enhanced carbon source.

Uranus and Neptune probably accreted in environments cold enough to allow condensation of methane or carbon monoxide clathrate; Saturn is a marginal case (Lewis 1974). One currently favored model for giant-planet formation, the nucleated collapse scenario (Mizuno 1980), would lead to incorporation of clathrate volatiles in the core with possibly substantial dissemination in the envelope. Earth-based data hint at a twofold to threefold methane enhancement in Saturn, and as much as twentyfold in Uranus and Neptune (Lutz, Owen, and Cess 1976). Under the simple assumption that the cores of these planets incorporated clathrate and then sufficient mass was removed to the envelope to produce the observed carbon enhancement, the noble gas enhancements can be calculated. Because of the smaller envelope-to-core ratio in these bodies, the amount of methane dredged from the core required to account for the observed enhancement is much smaller than for Jupiter: $>0.6 M_{\oplus}$ of methane for Saturn and $\leq 0.4 M_{\oplus}$ for Uranus and Neptune. The dredging process likely would not discriminate compositionally, allowing the use of Figure 22 (extrapolated for Uranus and Neptune) to determine noble gas enhancements in the envelope as a function of observed methane enhancement. The stability of clathrate under present temperature and pressure conditions in the giant planets was discussed by Miller (1961) - in particular, the possibility that the large $\mathrm{H}_{2}$ pressure and presence of other hydrocarbons might stabilize $\mathrm{CH}_{4}$ clathrate at the Jovian water-cloud level.

Finally, Titan's atmosphere may carry a record of the noble gas pattern calculated above. Since Titan is presumed to have accreted solely from condensed material, its volatile components were derived ultimately from the condensed solids or from gases entrapped in clathrate. The latter hypothesis would imply a distinctive noble gas pattern. Tied in with the clathrate hypothesis is the issue of the origin of the 1.5 bar $\mathrm{N}_{2}$ atmosphere (Lindal et al. 1983); measuring the argon-tonitrogen ratio is a potential diagnostic of the competing models.

The primitive $\mathrm{N}_{2}$ scenario (Owen 1982) derives the present atmospheric abundance of $\mathrm{N}_{2}$ directly from clathrate accreted during Titan's formation. The "secondary $\mathrm{N}_{2}$ " hypothesis uses the Atreya, Donahue, and Kuhn (1978) photochemical scheme for converting primordial ammonia condensed in the nebula into the $\mathrm{N}_{2}$ seen at present. The latter scenario requires a warm period early in Titan's history to permit the conversion to occur; an outline of a plausible scenario is given in Lunine and Stevenson (1982a). In either model, the $\mathrm{CH}_{4}$ and a number of other gaseous nebular constituents accrete as clathrate; up to $10^{25} \mathrm{~g}$ of $\mathrm{CH}_{4}$ can be accreted in this way. 


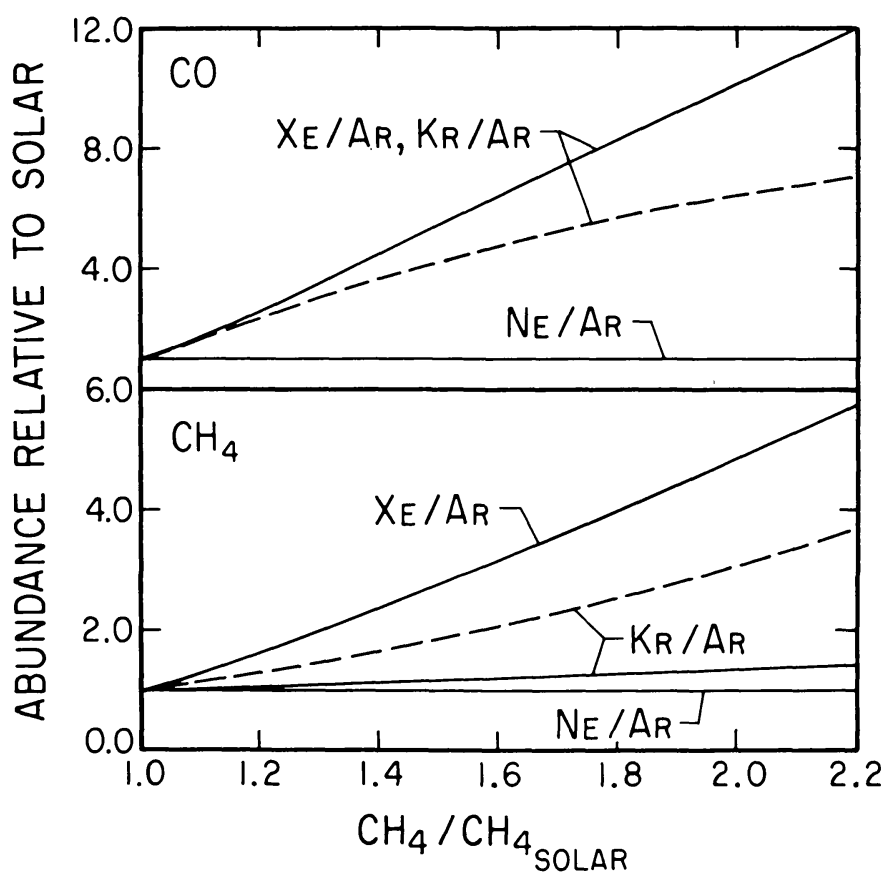

FIG. 22.- Predicted noble gas enhancements over solar abundance as a function of $\mathrm{CH}_{4}$ enhancement in Jovian atmosphere. Top panel assumes $\mathrm{CO}$-rich, and bottom $\mathrm{CH}_{4}$-rich, structure I clathrate. Note that essentially all $\mathrm{CO}$ brought into Jupiter is assumed converted to $\mathrm{CH}_{4}$. Formation temperature of $\mathrm{CO}$ clathrate is set at $60 \mathrm{~K}$, and that of $\mathrm{CH}_{4}$ clathrate at $100 \mathrm{~K}$. Dashed lines show enhancements for structure II clathrate.

In the secondary $\mathrm{N}_{2}$ hypothesis, up to $7 \%$ of the mass of Titan is accreted as condensed ammonia (the rock-to-ice ratio is $\sim 1.5$, modestly in excess of solar; Hunten et al. 1984), and up to several tens of bars of $\mathrm{N}_{2}$ could be produced photochemically at the surface. From our results above, it is clear that the amount of $\mathrm{N}_{2}$ brought into Titan in the primitive $\mathrm{N}_{2}$ hypothesis depends on the carbon composition of the nebula, i.e., on the ratio of $\mathrm{CH}_{4}$ to $\mathrm{CO}$. In the extreme limit where the primary carbon species is $\mathrm{CH}_{4}$, and all of the water ice is available for structure II clathration (no ammonia), $1.5 \times 10^{22}$ $\mathrm{g}$ of $\mathrm{N}_{2}$ could be brought into Titan by clathrate, or $\sim 2.6$ bars equivalent surface pressure. This is just marginally enough to explain the present atmosphere, especially allowing for escape of dissociated $\mathrm{N}_{2}$ with time (Strobel and Shemansky 1982). If the more likely case of structure $\mathrm{I} \mathrm{CH}_{4}$ clathrate is considered, only 1.4 bars of $\mathrm{N}_{2}$ is obtained. More interesting is the nitrogen to hydrocarbon ratio, which is estimated to be $\sim 0.2$ for the present Titan surface and atmosphere. This number includes the amount of hydrocarbons produced over the age of the solar system by photolysis of $\mathrm{CH}_{4}$, estimated from Yung, Allen, and Pinto (1984). The ratio for the $\mathrm{CH}_{4}$ clathrate is $5-9 \times 10^{-4}$. It is difficult to conceive of atmospheric escape or other processes that would remove (or sequester) more than $99 \%$ of the primitive methane incorporated in Titan while retaining (or outgassing) $90 \%$ of the entire primitive $\mathrm{N}_{2}$ budget in(to) the atmosphere.

More plausible perhaps is a nebula containing a mixture of $\mathrm{CO}$ and $\mathrm{CH}_{4}$. Increasing the $\mathrm{CO}-$ to- $\mathrm{CH}_{4}$ ratio in the gas decreases the $\mathrm{H}_{2} \mathrm{O}$ and $\mathrm{CH}_{4}$ abundance (the oxygen budget being assumed to remain fixed). Two effects occur: (1) the abundance in clathrate of $\mathrm{N}_{2}$ and $\mathrm{CO}$ relative to $\mathrm{CH}_{4}$ increases as the $\mathrm{CH}_{4}$ gas abundance goes down, and (2) the $\mathrm{CH}_{4}$ abundance eventually drops sufficiently that it is all enclathrated with excess water available to form $\mathrm{CO}-\mathrm{N}_{2}$ clathrate at lower temperatures. Because $\mathrm{CH}_{4}$ incorporates into clathrate much more readily than $\mathrm{CO}$ or $\mathrm{N}_{2}$, the $\mathrm{N}_{2}$ abundance in clathrate does not rise sharply until effect 2 occurs, when $90 \%$ of the carbon is in the form of CO. The preponderance of oxygen locked in $\mathrm{CO}$ implies a water abundance $\sim 0.4$ times that in a $\mathrm{CH}_{4}$-rich nebula. This would lead to an average density for Titan of $\sim 2.1 \mathrm{~g} \mathrm{~cm}^{-3}$, substantially higher than the observed value of $1.88 \mathrm{~g} \mathrm{~cm}^{-3}$. It would also lead to densities for the other Saturnian satellites of $\sim 1.7-1.8$ $\mathrm{g} \mathrm{cm}^{-3}$; their observed values are in the range $1.1-1.4 \mathrm{~g} \mathrm{~cm}^{-3}$. For this reason, we consider that the required extreme enrichment of $\mathrm{CO}$ relative to $\mathrm{CH}_{4}$ is implausible.

Figure 23 plots $\mathrm{N}_{2} / \mathrm{CH}_{4}$ and $\mathrm{CO} / \mathrm{CH}_{4}$ ratios in clathrate as a function of $\mathrm{CO} / \mathrm{CH}_{4}$ in the surrounding nebular gas $(T=80 \mathrm{~K})$. For a $\mathrm{N}_{2} / \mathrm{CH}_{4}$ (number) ratio of $\sim 0.25$ in clathrate, the CO-to- $\mathrm{N}_{2}$ abundance ratio in clathrate could be as high as $\sim 20$. The physical chemical properties of $\mathrm{CO}$ and $\mathrm{N}_{2}$ are sufficiently similar that this ratio should have been preserved in the Titan atmosphere. For this model to be acceptable then, some mechanism for destroying more than tens of bars of $\mathrm{CO}$ over the age of the solar system must be invoked. The photochemical mechanism of Samuelson et al. 


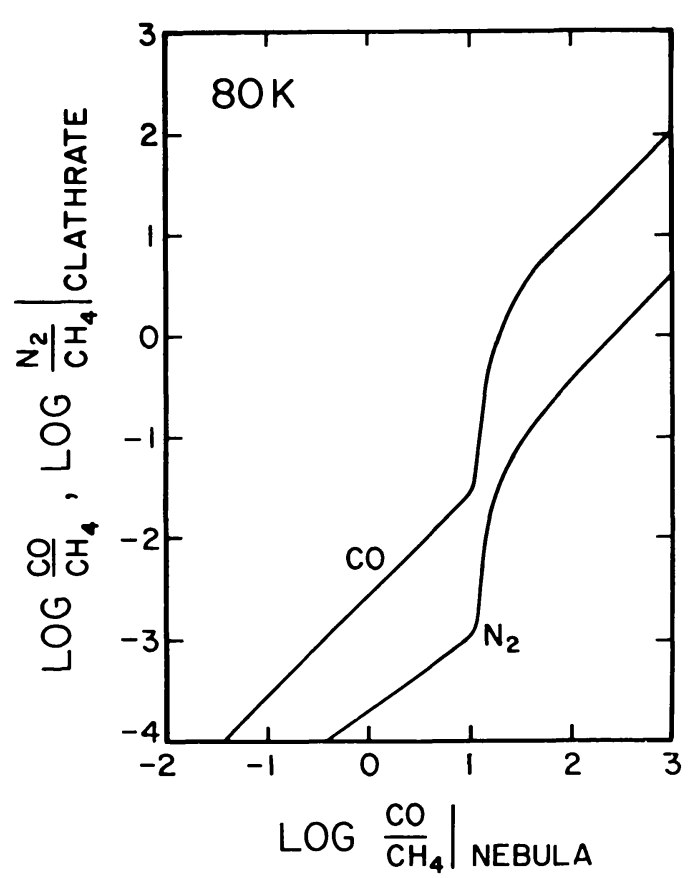

FIG. 23.- Ratio of $\mathrm{CO}$ and $\mathrm{N}_{2}$ to $\mathrm{CH}_{4}$ incorporated in clathrate as a function of CO-to- $\mathrm{CH}_{4}$ ratio in a solar composition nebula, at $80 \mathrm{~K}$. All nitrogen is assumed to be in the form of $\mathrm{N}_{2}$; total carbon-to-oxygen ratio in $\mathrm{H}_{2} \mathrm{O}, \mathrm{CO}$, and $\mathrm{CH}_{4}$ is solar.

(1983) can destroy 0.2 bars over the age of the solar system; this rate would have to be increased by $\geq 10-100$ to derive the present $\mathrm{CO} / \mathrm{N}_{2}$ ratio from that in clathrate.

One potentially diagnostic test of the competing models for $\mathrm{N}_{2}$ origin is measurement of the Ar-to- $\mathrm{N}_{2}$ abundance in the present atmosphere. If $\mathrm{N}_{2}$ is derived entirely from clathrate, then the Ar-to- $\mathrm{N}_{2}$ ratio in Titan's atmosphere would be $\sim 10^{-2}$ to $10^{-1}$. The molecular properties of $\mathrm{Ar}$ and $\mathrm{N}_{2}$ are such that their outgassing histories plausibly could be similar; photochemical and charged-particle destruction of $\mathrm{N}_{2}$ in the atmosphere might reduce $\mathrm{N}_{2}$ by $20 \%$ over the age of the solar system (Strobel and Shemansky 1982; Yung, Allen, and Pinto 1984). If the $\mathrm{N}_{2}$ were photochemically derived, and the primitive $\mathrm{N}_{2}$ abundance relative to carbon were negligible (as for $\mathrm{CH}_{4}$ clathrate), then $\mathrm{Ar} / \mathrm{N}_{2}$ would depend on the amount of $\mathrm{N}_{2}$ produced photochemically and on the Ar outgassing and escape history. Define $\chi \equiv \mathrm{Ar}$ abundance relative to $\mathrm{CH}_{4}$ (and $\mathrm{CH}_{4}$-produced hydrocarbons) for the present Titan surface, divided by initial $\mathrm{Ar} / \mathrm{CH}_{4}$ ratio in pure $\mathrm{CH}_{4}$ clathrate. If all available $\mathrm{Ar}$ were outgassed and retained in the present atmosphere $(\chi \sim 300), \operatorname{Ar} / \mathrm{N}_{2} \sim 10^{-2}$ to $10^{-1}$; if the $\mathrm{Ar}$ and $\mathrm{CH}_{4}$-derived hydrocarbon outgassing and loss histories have been similar, $\chi=1$ and $\mathrm{Ar} / \mathrm{N}_{2} \sim 10^{-4}$. Thus, a low value of $\mathrm{Ar}$ in the Titan atmosphere would suggest $\mathrm{N}_{2}$ derived not from clathrate but from primitive $\mathrm{NH}_{3}$; a large Ar value would be ambiguous. Voyager data require no Ar and yield an upper limit of 0.12 (Lindal et al. 1983).

The above test will be affected only slightly by the presence of an ocean on Titan's surface; a kilometer-deep ethane- methane ocean has been proposed based on Voyager data (Lunine, Stevenson, and Yung 1983). Figure 24 (top) plots $\mathrm{Ar} / \mathrm{N}_{2}$ in ocean and atmosphere, assuming the atmospheric $\mathrm{Ar} / \mathrm{N}_{2}$ is fixed at 0.1. Figure 24 (bottom) plots $\mathrm{Kr} / \mathrm{Ar}$ in the atmosphere as a function of ocean depth, assuming $\mathrm{Kr} / \mathrm{Ar}$ in the ocean and the atmosphere is that expected in clathrate, $\sim 0.1$. Although the solubility of $\mathrm{Kr}$ in hydrocarbons is substantially larger than that of Ar, even a deep ocean would preserve a nonsolar $\mathrm{Kr} / \mathrm{Ar}$ atmospheric abundance, if the total ocean and atmosphere ratio is nonsolar. Similar results are expected for $\mathrm{Xe} / \mathrm{Ar}$ although no solubility data on $\mathrm{Xe}$ in hydrocarbons apparently exist.

In conclusion, an enhanced heavy noble gas and depleted neon abundance are signatures of primordial incorporation of these constituents in Titan as clathrate. The $\operatorname{Ar} / \mathrm{N}_{2}$ ratio in the present atmosphere is potentially a test of the origin of the $\mathrm{N}_{2}$ making up the bulk of the atmosphere. The rather modest enhancement of rock/ice in Titan relative to the cosmic abundance, and the current lack of a means for almost completely destroying large amounts of $\mathrm{CO}$ in the Titan atmosphere both argue, however, against a primordial, clathrate origin for $\mathrm{N}_{2}$ in Titan.

We now examine whether double occupancy of cages by $\mathrm{H}_{2}$ and $\mathrm{CH}_{4}$ is an important source of $\mathrm{H}_{2}$ in solar system objects. Double occupancy by $\mathrm{H}_{2}$ of $\mathrm{H}_{2}$-occupied cages is not important since the number of cages singly occupied by $\mathrm{H}_{2}$ is small. The fraction of $\mathrm{CH}_{4}$-occupied cages also containing. $\mathrm{H}_{2}$ is

$$
d_{M-\mathrm{H}}=\frac{q_{M \mathrm{H}} C_{\mathrm{H}} P_{\mathrm{H}} C_{M} P_{M}}{C_{M} P_{M}}=q_{M \mathrm{H}} C_{\mathrm{H}} P_{\mathrm{H}},
$$

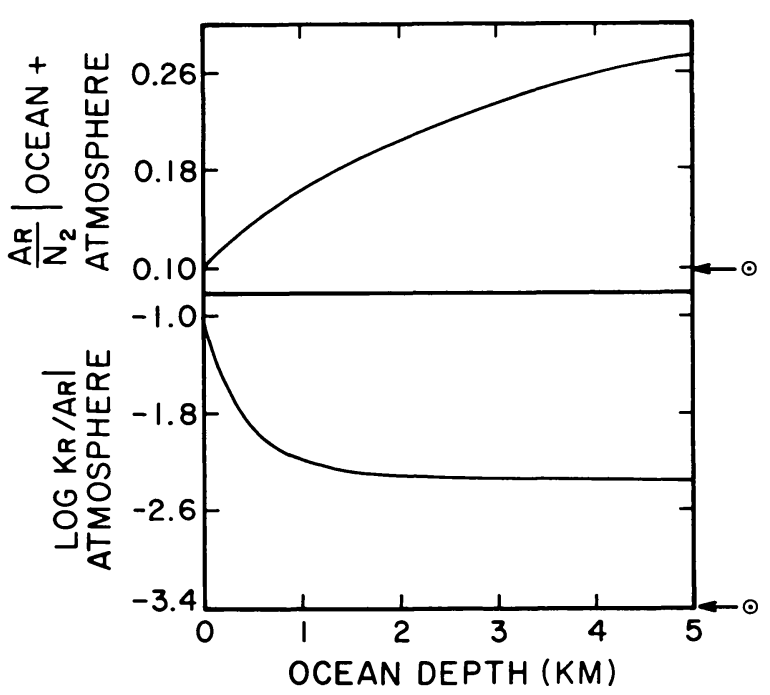

FIG. 24.-Abundance patterns expected in Titan atmosphere and hypothetical hydrocarbon ocean predicted from solubility considerations, as a function of ocean depth. Top panel plots Ar-to- $\mathrm{N}_{2}$ ratio in ocean plus atmosphere assuming atmospheric ratio of 0.1 , roughly the Voyager upper limit (Lindal et al. 1983). Bottom panel shows ratio of $\mathrm{Kr}$-to-Ar abundance in atmosphere assuming ratio in ocean plus atmosphere is 0.1 , roughly that predicted from $\mathrm{CH}_{4}$-rich clathrate model. Arrows indicate solar values (assuming all nitrogen is in the form of $\mathrm{N}_{2}$ ). Note log scale in bottom panel. 
where $P_{M}, P_{\mathrm{H}}$ are ambient $\mathrm{CH}_{4}$ and $\mathrm{H}_{2}$ gas pressures and $C_{M}, C_{\mathrm{H}}$ are large-cage Langmuir constants (the doubleoccupancy probability in the small cages is zero). Plausible Saturnian nebular models give $P_{\mathrm{H}} \sim 0.1$ bars at $100 \mathrm{~K}$ (Prinn and Fegley 1981; see also Lunine and Stevenson 1982 b). Equation (58) then becomes, with structure I $C_{\mathrm{H}}$ calculated using the L-J potential parameters, $d=0.3 q_{M \mathrm{H}}$. Hence, $d=$ $10^{-2}$ to 0 for the range of $q_{M \mathrm{H}}$ calculated in $\S$ III. The structure II large cage has $q_{M \mathrm{H}} \sim 5$ times larger, but makes up only one-third of the total number of cages. Double-occupancy results are therefore quite similar for either clathrate form. The upper bound is potentially a substantial amount of $\mathbf{H}_{2}$, which, incorporated into icy satellites, may have consequences for subsequent outgassing histories.

For example, if Titan incorporated most of its water ice as methane clathrate, then the total $\mathrm{CH}_{4}$ mass incorporated would be $\sim 0.06 \times 1.4 \times 10^{26} \mathrm{~g}, \sim 1 \times 10^{25} \mathrm{~g}$. If $\mathrm{H}_{2}$ "doubleoccupies" the cages to the maximum extent calculated above, the total $\mathrm{H}_{2}$ mass incorporated in Titan would be $\sim 10^{22} \mathrm{~g}$, or $10^{4}$ times the inferred amount in the present atmosphere (Hunten et al. 1984). The mass of $\mathrm{H}_{2}$ which has been produced photochemically over the age of the solar system is $\sim 5 \times 10^{21} \mathrm{~g}$ (Yung, Allen, and Pinto 1984), i.e., comparable to our maximum speculative primordial source.

Neon, lower in solar abundance than $\mathrm{H}_{2}$ by a factor of $10^{4}$, but with comparable L-J parameters, could have $d_{M-\mathrm{Ne}} \leq$ $10^{-6}$. Although still a trace amount, this could be the primary source of $\mathrm{Ne}$ in clathrate. The double-occupancy enhancements of $\mathrm{H}_{2}$ and $\mathrm{Ne}$ in primordial clathrate are shown in Figures 18 and 19. The low Langmuir constant for helium relative to hydrogen yields $d_{M-\mathrm{He}} \leq 10^{-5}$, although the smaller value of $\sigma$ for helium versus hydrogen may increase $q_{M-\mathrm{He}}$ and $d_{M-\mathrm{He}}$ by $\sim 3$ or so. Helium is thus substantially depleted relative to solar abundance.

We now briefly consider the relevance of clathrates to cometary composition and phenomena. An extensive literature exists in this area (see, e.g., Delsemme 1983), and two points must be emphasized: (1) there are no compelling observational data strongly for or against a primordial clathrate component to cometary volatiles, and (2) no physical cometary phenomena observed require (or rule out) the presence of clathrates in comets. Our work adds a theoretical argument against primordial clathrate being a primary component of cometary nuclei, if indeed the formation region of these bodies was in the outer (trans-Neptunian) solar nebula: kinetic inhibition of clathrate formation would be expected under conditions in the outer solar nebula. If comets initially incorporated clathrates, the extremely long time scale for diffusion of gas through ice grains implies that this material may still exist in the nucleus.

The distinctive noble gas systematics predicted above in the scenario for carbon enrichment in Jupiter provides a somewhat indirect and tenuous test of the presence of clathrate in comets, if it is supposed that the source of the enrichment was infalling cometary material from the outer solar system. A more direct test would be mass spectrometric measurements of noble gas abundances in the gas surrounding a comet nucleus.

In closing this section it is worth considering again the work of Sill and Wilkening (1978), with regard to contamination of terrestrial atmospheres by infalling planetesimal debris con- taining clathrate. They point out the distinctive noble gas signature of clathrates, and the resemblance of $\mathrm{Xe} / \mathrm{Ar}$ and $\mathrm{Kr} / \mathrm{Ar}$ to terrestrial atmosphere noble gas signatures (Fig. 21). The terrestrial nitrogen, carbon, neon, and water budget in their model must, however, be augmented by other sources. This conclusion is not surprising considering the complexity of accretion and evolution processes in large bodies and the requirement of transporting clathrate, as solar-orbiting cometary debris, into the inner solar system where temperatures during accretion would have been too high to condense it directly. Clathrate may have been much more important in determining the volatile budget in the outer solar system (with the proviso of kinetic limitations) than in terrestrial planets. It is still necessary to consider carefully physical processes in bodies which may segregate volatiles and further evolve abundance patterns, as illustrated in the next two sections.

\section{b) Application to Titan Accretion and Cooling History}

In this section, two applications of the phase diagram shown in Figure 8 are outlined: modeling of the primordial atmosphere of Titan during accretional heating and postaccretional cool down. Both of these applications involve extensive modeling of physical processes which is beyond the scope of this paper. Much of the effort to fully explore these models is work in progress. Here we will emphasize the role of clathrate thermodynamics.

The accretional model is motivated by the need to understand the initial conditions of the atmosphere and volatile budget of Titan. The possible implications of the extreme range of accretion models, from cold (where the impact velocity of planetesimals and, hence, surface temperatures are low) to hot (high velocity, high temperature), are discussed in Hunten et al. (1984). The high mass of Titan and the high gas pressures in Saturnian nebular models motivate the so-called gaseous accretion model. The physics of this model and its application to the Galilean satellites are given in Lunine and Stevenson (1982b). Briefly, the satellite, forming in a highpressure ( $\sim 0.1$ bars) nebula, gravitationally "captures" a solar composition gaseous envelope, which is optically thick in all but the earliest stages of accretion. Planetesimals falling through the envelope are slowed by gas drag, disseminating a portion of their kinetic energy and volatile mass in the envelope. The temperature structure in this optically thick envelope is adiabatic because of the large accretional energy. The highest surface temperatures are obtained under the assumption that the planetesimal mass is disseminated in small pieces, allowing thermal equilibration between the infalling planetesimal mass and atmosphere; this is the limiting case we consider here. The convective temperature structure of the atmosphere is modified by the latent heat release of freezing and condensing volatiles, as given in equation (22) of Lunine and Stevenson (1982b). For Titan, the primary volatiles in the model are ammonia hydrate and methane clathrate. (We assume that the entire solar abundance of nitrogen is in the form of ammonia. The abundance of other volatiles contained in the clathrate is too small to affect the thermodynamics of the system.) So long as the abundance of methane is not a limiting factor (see below), the atmosphere and, hence, surface 
are constrained to be methane-saturated with respect to clathrate. On a plot of methane pressure versus temperature, such as Figure 25 (a portion of the phase diagram of Figure 8), methane pressure as a function of temperature through the atmosphere runs along the dissociation pressure curve. Hence, as the satellite mass grows and the atmosphere "deepens," the surface temperature must also run along this curve, and we can identify the coexisting surface phase assemblages at each point during accretion.

During the late stages of accretion the temperature-pressure curve runs off the clathrate dissociation pressure line at a point in this atmosphere at which incoming planetesimals can no longer supply enough methane mass to maintain the clathrate-saturated pressure. Temperatures increase more rapidly toward the surface because of $(a)$ the absence of the clathrate-saturated adiabat and $(b)$ the low specific heat of the now dominant $\mathrm{CH}_{4}$ and $\mathrm{NH}_{3}$ gaseous constituents (as opposed to $\mathrm{H}_{2}, \mathrm{He}$ ). The point at which the atmosphere departs from the dissociation curve in Figure 25 should be regarded as schematic. This calculation demonstrates that most of the $\mathrm{CH}_{4}$ accreted onto Titan is liberated into the primordial atmosphere, rather than sequestered as clathrate. The methane affects the subsequent evolution of the atmosphere, as discussed below.

Similarly, application of the phase diagram to the postaccretional cool down of Titan's atmosphere permits the iden-

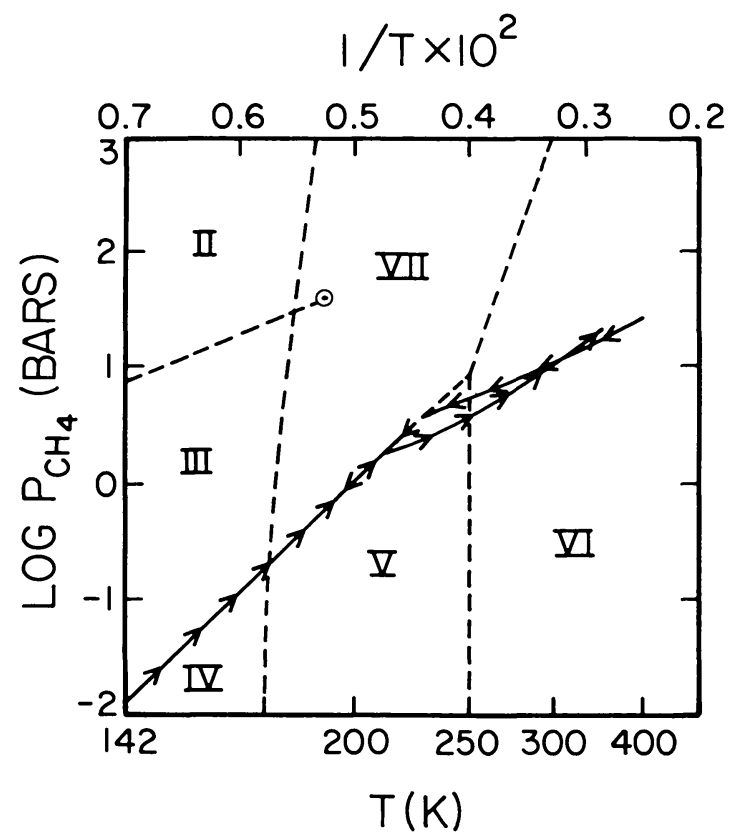

FIG. 25. - Water-ammonia-methane phase diagram $\left(x_{a}=0.15\right)$, reproduced from Fig. 8, illustrating application to early Titan models. Arrows superposed show schematically the temperature-pressure path taken by the base of Titan's atmosphere during gaseous accretion, and subsequent cooling driven by rapid, adiabatic escape. The atmosphere is assumed to be in contact with an ammonia-water solid or liquid surface as appropriate. Arrows on the phase boundaries III-IV and V-VII indicate the base of the atmosphere is saturated with respect to methane clathrate formation; arrowed paths in V and VI imply too little atmospheric methane to form clathrate at the specified temperature. tification of the effect of surface phase assemblages on the cooling history. Although detailed modeling of mechanisms for loss of gas and cooling has not been done, a simple adiabatic cooling model is adequate to illustrate use of Figure 25. Adiabatic cooling of an atmosphere would be expected if an energy source (such as solar EUV heating) were available to remove gas rapidly (see Watson, Donahue, and Walker 1981); if the lower atmosphere were an optically thick gray medium, then an adiabatic temperature profile

$$
\frac{T}{T_{0}}=\left(\frac{P}{P_{0}}\right)^{\gamma-1 / \gamma}
$$

is appropriate in that region. Here $\gamma$ is the ratio of gas specific heats, and $T_{0}$ and $P_{0}$ are temperature and pressure at optical depth unity in the atmosphere. We choose $T_{0}=90 \mathrm{~K}, P_{0}=0.1$ bars in a pure methane atmosphere for illustrative purposes, and in Figure 25 we plot the adiabat given by equation (59) and values of $\gamma$ determined from Goodwin (1974). The choice of $T_{0}$ and $P_{0}$ would be appropriate for formation of methane clouds as the opacity source along with pressure-induced gas opacity, the adiabat moving away from pure methane saturation with increasing temperature. Note also that although water and ammonia vapor are present in the atmosphere as saturated vapor in equilibrium with the surface, their vapor pressures are so small at temperatures of interest that the gas may be considered to be pure methane.

By the above assumptions, the adiabat plotted also represents the change of surface pressure with temperature. If we start the atmosphere at some high temperature and pressure on the adiabat (59), with the methane pressure well below clathrate saturation (i.e., in region IV, V, or VI), and allow it to cool, the pressure-temperature path must intersect the clathrate dissociation pressure curve and cross into region III or VII. Our choice of adiabat crosses into region VII, and clathrate begins to form at $\sim 225 \mathrm{~K}$, the surface phases being water ice in coexistence with ammonia-water liquid and methane gas. Although higher levels in the atmosphere will also be in the clathrate-stable region, the lack of water precludes clathrate formation. The near-surface atmosphere must now move along the dissociation curve in Figure 25, with the production of clathrate from methane gas and surface water ice causing the gas pressure to drop more sharply with temperature than on the adiabat. (Latent heat release due to clathrate formation may buffer the temperature drop and increase cooling times, but the surface must move along the dissociation curve.) The dropout of the atmosphere as clathrate can continue provided the previously formed clathrate sinks. Although the densities of the ammonia water and the methane clathrate are inadequately known (for ammonia water: International Critical Tables 1928, p. 59; Hildenbrand and Giauque 1953; for clathrate: Byk and Fomina 1968; Kvenvolden and McDonald 1982), the clathrate may be slightly denser, especially if molecules heavier than $\mathrm{CH}_{4}$, such as $\mathrm{N}_{2}$, are also incorporated. At $172 \mathrm{~K}$, eutectic freezing of the surface occurs, and a layer of clathrate in contact with the atmosphere forms over the ice, kinetically inhibiting further clathrate formation at a methane pressure of 0.18 bars. 
The scenario becomes more interesting if, during the warm period after accretion, several tens of bars of $\mathrm{N}_{2}$ were photolytically produced from gaseous $\mathrm{NH}_{3}$ (Atreya, Donahue, and Kuhn 1978). An outline of a cooling scenario involving $\mathrm{N}_{2}$ photolysis is given in Lunine and Stevenson (1982a). Consideration of the dissociation pressure curve for a mixed $\mathrm{N}_{2}-\mathrm{CH}_{4}$ clathrate suggests that the near-surface portion of such an atmosphere begins producing clathrate at $\sim 200 \mathrm{~K}$. As the surface cools, moving along the dissociation pressure curve, the amounts of $\mathrm{N}_{2}$ and $\mathrm{CH}_{4}$ incorporated in the clathrate differ; hence, the composition of the atmosphere changes during the cooling. Using structure I Langmuir constants for $\mathrm{N}_{2}$ and $\mathrm{CH}_{4}$ computed in $\S \mathrm{III}$, we calculate gas pressures of the two guest molecules and relative abundances in the clathrate at a given temperature $T$. Assuming the clathrate sinks, permanently sequestering the encaged $\mathrm{N}_{2}$ and $\mathrm{CH}_{4}$, we then self-consistently compute the new equilibrium pressures and incorporation factors of $\mathrm{CH}_{4}$ and $\mathrm{N}_{2}$ at a lower temperature $T-\Delta T$, repeating the process until the eutectic temperature of $172 \mathrm{~K}$ is reached. To facilitate the calculation we use a convenient expression for the equilibrium pressures $P_{i}$ derived by Miller (1961) from equations (1) and (2):

$$
\sum_{i} \frac{P_{i}}{P_{i}^{0}}=1,
$$

where $P_{i}^{0}$ is the dissociation pressure for pure species $i$. For $\mathrm{N}_{2}$ and $\mathrm{CH}_{4}, P_{i}$ from equation (60) agrees with that from the full expression to $20 \%$. The greater propensity of $\mathrm{CH}_{4}$ to incorporate in clathrate results in enrichment of the atmosphere in $\mathrm{N}_{2}$ with decreasing temperature. Although results do depend on initial gas abundances, for comparable initial values of $\mathrm{N}_{2}$ and $\mathrm{CH}_{4}$ and total pressure of order $\sim 10$ bars, the final abundances of $\mathrm{N}_{2}$ and $\mathrm{CH}_{4}$ at $172 \mathrm{~K}$ are similar to those in the present atmosphere of Titan, i.e., several bars of $\mathrm{N}_{2}$ and less than 0.5 bars of $\mathrm{CH}_{4}$. Figure 26 plots total pressure and methane mole fraction in structure I clathrate and atmosphere as a function of temperature for the model case of equal starting abundances of $\mathrm{N}_{2}$ and $\mathrm{CH}_{4}$ in the atmosphere. The results are qualitatively similar if structure II clathrate were formed. Although the current atmospheric value of $\mathrm{CH}_{4}$ (Lindal et al. 1983) may be only a small fraction of the surface hydrocarbon budget (Lunine, Stevenson, and Yung 1983), it is intriguing that the amount of $\mathrm{N}_{2}$ corresponds so closely to the present-day value. Additional work must be done to better characterize the physical processes involved in the cooling before firm conclusions can be drawn; in particular, the implications of new data on the ammonia-water phase diagram (Johnson, Schwake, and Nicol 1985) must be incorporated in analyzing the effect of the ocean on the atmosphere.

Additional calculations along the above lines have been done including Ar and other noble gases as well as $\mathrm{N}_{2}$ and $\mathrm{CH}_{4}$. The $\mathrm{Ar}$ tends to partition with $\mathrm{N}_{2}$ in the cooling atmosphere, while $\mathrm{Kr}$ and $\mathrm{Xe}$ partition strongly into the "oceanic" clathrate. The differing solubilities for various atmospheric constituents and the temperature dependence of the solubility in the $\mathrm{NH}_{3}-\mathrm{H}_{2} \mathrm{O}$ solution also modify the atmospheric composition and cooling process in such models.

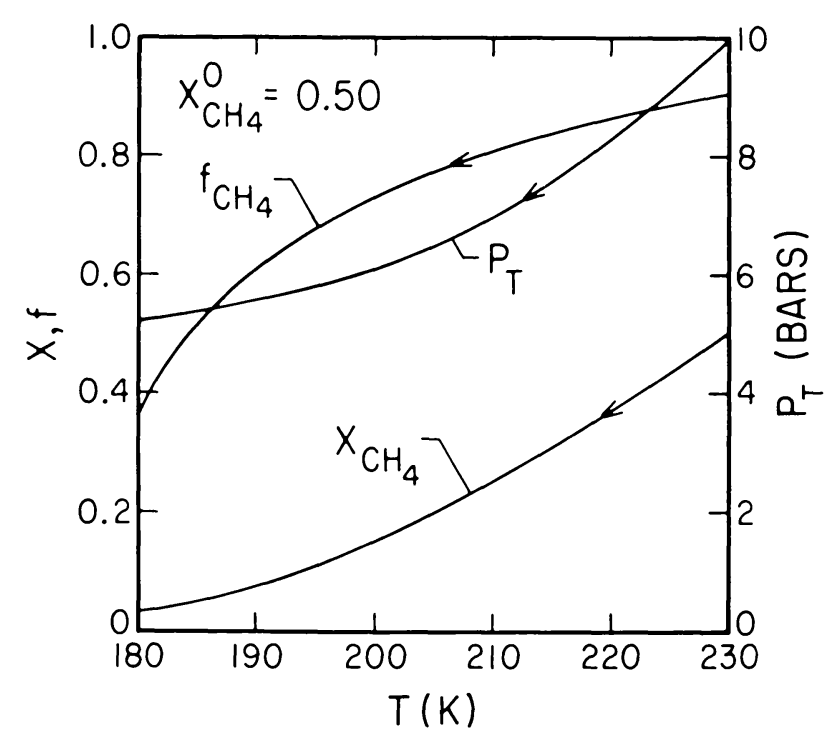

FIG. 26.-Composition of a cooling $\mathrm{N}_{2}-\mathrm{CH}_{4}$ gas in coexistence with clathrate as a function of temperature. The gas in contact with water ice is assumed to start at 10 bars total pressure $\left(P_{T}\right)$ and methane fraction $x_{\mathrm{CH}_{4}}=0.5$; under these conditions at $230 \mathrm{~K}$ clathrate formation ensues, and the resulting composition and pressure changes as a function of temperature are plotted. A well-mixed, isothermal atmosphere is assumed. Here, $f_{\mathrm{CH}_{4}}$ is $\mathrm{CH}_{4}$ composition (relative to $\mathrm{CH}_{4}, \mathrm{~N}_{2}$ ) in clathrate. The calculations are applicable to modeling of a cooling $\mathrm{N}_{2}-\mathrm{CH}_{4}$ Titan atmosphere in contact with a freezing ammonia-water ocean at its base; the clathrate is assumed to sink, allowing further clathrate formation to occur. The temperature decrease is then driven by the loss of atmosphere to clathrate.

Details of ongoing work are beyond the scope of this paper; here we have demonstrated the necessity of understanding clathrate thermodynamics in the presence of ammonia (for methane alone as well as with other guest molecules of cosmochemical interest) in constructing satellite evolution models.

\section{c) Application to Satellite Internal Evolution}

Oh that delightful engine of her thoughts That blabbed them with such pleasing eloquence Is torn from forth that pretty hollow cage, Where like a sweet melodious bird it sung Sweet varied notes, enchanting every ear!

(Titus Andronicus, act 3, sc. 1, line 26)

We briefly outline application of Figure 10 to the stability of clathrate in Titan and other icy satellites.

Accretion scenarios for large satellites (Lunine and Stevenson $1982 b$; Hunten et al. 1984) predict an initially undifferentiated rock-ice core underlying a differentiated mantle, the relative sizes of the regions being dependent on the choice of initial formation conditions, accreting volatiles, and details of the accretional model. Figure 27 shows one possible configuration for Titan at the close of accretion, assuming ammonia hydrate and methane clathrate were present in planetesimals. Based on the results of Figure 10, methane clathrate is unstable at $P \gtrsim 11-14$ kilobars and would dissociate as soon as core pressures exceeded this range during accretion. At the temperatures predicted for the core, methane 
TITAN AT CLOSE OF ACCRETION

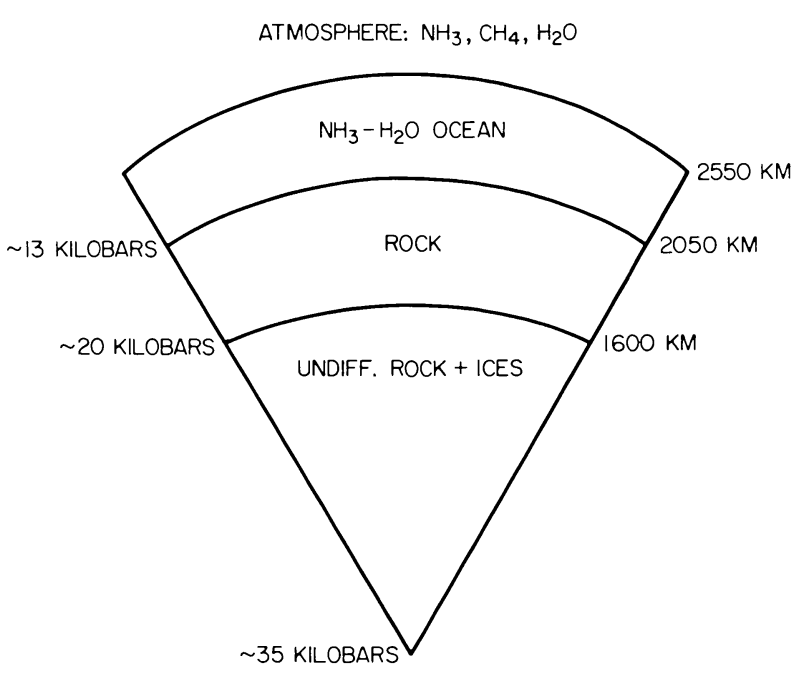

FIG. 27.- Schematic model of the internal structure and atmospheric composition of Titan immediately after accretion. Radii are indicated on the right side of the figure; approximate pressures on the left. Subsequent evolution includes overturn of undifferentiated core, which is replaced by overlying rock layer (see Friedson and Stevenson 1983).

would likely remain a solid, buoyant relative to water ice, and could move upward during a postulated later overturn of core and mantle (the latter process is described in Friedson and Stevenson 1983). The subsequent history of the methane is dependent on the amount of methane already in the mantle, temperatures in that region, and the phase relationships derived above. The issue to be addressed is whether the total methane and methane-derived hydrocarbon mass inferred to be on the surface of Titan today (Yung, Allen, and Pinto 1984; Lunine, Stevenson, and Yung 1983) could be derived from an internal source such as a primordial core. Measurement of the volume of the newly identified high-pressure ammonia-dihydrate phase (Johnson, Schwake, and Nicol 1985) will allow calculation of the clathrate stability field at $x_{\mathrm{H}_{2} \mathrm{O}}<$ 0.85 , which has important implications for the internal evolution outlined above.

Also bearing on this issue is the occupancy factor of clathrate as a function of pressure. At high pressures ( $T>100$ bars) $y$ as defined in equation (2) is $\approx 1$. As pressure decreases, $y$ decreases; at $230 \mathrm{~K}$ and 5.61 bars $y=0.90$ in the structure I small cage. Although this is a small effect, the amount of methane and methane-derived products inferred to be on Titan's surface is also a small fraction $(<1 \%)$ of the maximum which could have been incorporated in Titan as clathrate; in fact, it is only $\sim 10 \%$ of the amount estimated to be in the primordial core of Figure 27. Whether methane-rich clathrate can work its way to the near-surface environment and release methane requires modeling of physical processes in conjunction with the thermodynamic modeling presented here.

Pressures are less than several kilobars and temperatures are less than $300 \mathrm{~K}$ in the icy intermediate-sized Saturnian satellites (Stevenson 1982a); similar conditions may be obtained in the icy Uranian satellites (Stevenson 1984). $\mathrm{CH}_{4}$, $\mathrm{N}_{2}$, and/or CO clathrates are therefore thermodynamically stable in these satellites according to Figure 10. An important point is that the clathrate is stabilized by confining hydrostatic pressures and not by coexistence of a guest molecule (i.e., $\mathrm{CH}_{4}$ ) pure phase (which could slowly diffuse out of the body). Only in a near-surface, porous regolith would diffusion and escape of guest molecules occur, leading to depletion of clathrate in this outermost, kilometer-deep (Hartmann 1973) regime. The diffusion process is sufficiently slow that impactgenerated release of methane from clathrate may be more important. However, both impacts and charged-particle bombardment (Cheng and Lanzerotti 1978) of the methane may then produce dark polymeric material.

\section{SUMMARY AND CONCLUSIONS}

We have undertaken an analysis of the thermodynamics and kinetics of clathrate formation under conditions of interest to outer solar system studies. In particular, we have used a statistical mechanical model of clathrate formation from van der Waals and Platteeuw (1959) along with laboratory data to systematically calculate dissociation pressures over a wide range of temperatures for guest molecules of cosmochemical interest. We have predicted the dissociation pressure-temperature curve for $\mathrm{CO}$ clathrate, which has not been examined experimentally, as well as calculated the degree of double occupancy of cage sites by small molecules such as $\mathrm{H}_{2}$. The above results were then used to determine the abundance pattern of volatiles entrapped in clathrate in solar composition gaseous nebulae postulated to be source regions of planet and satellite accretion. Predictions of nonsolar gas abundances in giant-planet and satellite atmospheres were made, and are potentially testable (Galileo probe measurements of Jupiter's atmosphere, for example). The issue of kinetics of clathrate formation in such nebulae was addressed, and it was concluded that collisional stirring of planetesimals may be required to ensure substantial clathration of the ice.

The phase diagrams of methane clathrate and $\mathrm{N}_{2}$ clathrate over the entire pressure-temperature range of stability were calculated, and the effect of ammonia was considered. Experimental data to verify the predicted diagrams do not exist. The utility of such diagrams in modeling the evolution of large satellites such as Titan was also demonstrated.

We conclude with a list of recommended observations and laboratory investigations which would test our predictions and resolve remaining uncertainties in the work. Such data are of crucial importance in understanding the role of clathrate hydrate in satellite and planetary evolution in the outer solar system.

1. Measure dissociation pressure and structure as a function of temperature of clathrates of $\mathrm{CO}, \mathrm{N}_{2}, \mathrm{CH}_{4}, \mathrm{Ar}, \mathrm{Kr}, \mathrm{Xe}$, $\mathrm{Ne}, \mathrm{H}_{2}$, down to $T \sim 50 \mathrm{~K}$. Examine the effect of the presence of amorphous ice on clathrate formation. In the cases of $\mathrm{H}_{2}$ and $\mathrm{Ne}$, identification of cage double occupancy is useful.

2. Map out phase diagram of clathrate formation at low pressure in presence of ammonia hydrate compounds and ammonia-water solution.

3. Map out stability regions of $\mathrm{CH}_{4}, \mathrm{~N}_{2}$, and $\mathrm{CO}$ clathrate up to $P \gtrsim 15$ kilobars and $T \gtrsim 320 \mathrm{~K}$. Measure the effect of ammonia on the resulting phase diagram. Determination of the solubility of methane in water and ammonia-water solu- 
tions at high $\left(10^{4}\right.$ bars $)$ pressures is also crucial to satellite evolution studies.

4. Study the kinetics of clathrate formation in the laboratory, with emphasis on diffusion time scales of gas through ice to form clathrate. Elucidation of the process whereby agitation of ice and gas facilitates clathrate formation would be helpful; i.e., is the exposure of fresh ice, or vaporization and recondensation of water more important for the formation of clathrate?

5. Measure the density of clathrate containing various proportions of $\mathrm{CH}_{4}$ and $\mathrm{N}_{2}$ at low pressure; also, the compressibility of clathrate at high $\left(10^{4}\right.$ bars $)$ pressure. Measure the density of high-pressure ammonia-water phases and map out their regions of stability. These properties are important to satellite evolution studies.

6. Study rheological and thermal properties of clathrate hydrate under a range of pressures. Indirect evidence for a larger number of defects in clathrate structure than in ice I (Davidson and Wilson 1963) and limited studies of $\mathrm{H}_{2} \mathrm{~S}$ clathrate (Pinder 1964) suggest rheology distinct from ice I. The low thermal conductivity reported by Cook and Leaist (1983) and Stoll and Bryan (1979) should be confirmed. These properties are important to thermal models of satellites which may contain clathrate.

7. Study the spectroscopic detectability of clathrate and its products. Smythe (1975) studied near-IR reflection spectra of clathrate; study of the visible and IR properties of polymeric products of $\mathrm{CH}_{4}^{-}, \mathrm{N}_{2^{-}}$, and CO-rich clathrate produced by particle bombardment of surfaces could be crucial to identifying satellites whose interiors may contain clathrate. (Are the products detectably different from those produced from the pure $\mathrm{CH}_{4}, \mathrm{~N}_{2}$, or $\mathrm{CO}$ frosts?)

We thank Stanley Miller for fruitful and helpful discussions, and D. W. Davidson for a critical review of the manuscript. This work was supported by NASA grant NAGW-185.

\section{APPENDIX}

To determine the high-pressure stability field of clathrate hydrate, quantitative evaluation of $\Delta \mu^{\beta}$ and $f_{c}$ as a function of temperature and pressure is required. We rewrite the pressure dependence of $\Delta \mu^{\beta}$ as

$$
\Delta \mu^{\beta}(T, P)=\Delta \mu^{\beta}\left(T, P_{0}\right)+\sum_{i} \int_{P_{\imath-1}}^{P_{\imath}} \Delta V_{i}^{\beta}(T, P) d P,
$$

where we have broken up the pressure integral into intervals corresponding to phases of water encountered along the isothermal path. Within each phase, however, $\Delta \mu^{\beta}$ is still a function of pressure. Equations for density as a function of temperature and pressure for water ice are given in Lupo and Lewis (1979); these are fits to data in previous sources. Several of these sources were checked independently (Bridgeman 1913, 1937; Dorsey 1940, p. 212) to verify that at high ( $\sim 10$ kilobars) pressure the Lupo and Lewis fits agree with the data to within $\sim 1 \%$. Phase boundaries for the water system were determined from Eisenberg and Kauzmann (1969, p. 93) and Lupo and Lewis (1979).

The compressibility of the clathrate cage, on the other hand, is not well known. The existing data on sound velocity in clathrate give a value within $20 \%$ of that of ice I, and which is only weakly dependent on type of guest molecule (Pearson et al. 1983; Stoll and Bryan 1979). Also, the volume thermal expansivity of clathrates has been assumed to be similar to that of ice I in previous studies (Pearson et al. 1983). We therefore assume

$$
K^{\beta}=\left.\frac{1}{V} \frac{d V}{d P}\right|_{T} ^{\text {clathrate }}=\left.\frac{1}{V} \frac{d V}{d P}\right|_{T} ^{\text {ice I }}
$$

where the ice I value is given in Lupo and Lewis (1979). A calculation by Whalley (1980) is consistent with equation (A2). We use $K^{\beta}=$ constant. We also assume that the clathrate undergoes no structural phase transitions in the pressure range under consideration. Although no experimental data exist to justify these assumptions, and there is some weak evidence supporting the existence of a metastable high-pressure modification of clathrate (Ross and Andersson 1982), we adhere to the simplest approximation here. Varying $K^{\beta}$ for the clathrate (for example, setting it equal to that of the coexisting high-pressure form of ice) below $273 \mathrm{~K}$ changes $\Delta \mu^{\beta}$ in the high-pressure regime by $\sim 1-3 \%$ and the pressure of the clathrate phase boundary by $10-20 \%$, which is not crucial. In the liquid-water region, however, the phase boundary pressure is very sensitive to the choice of $K^{\beta}$. As indicated above, however, data in the "low" ( $<4$ kilobars) region as well as the results in the high-pressure ice field constrain the clathrate stability boundary in the liquid-water field, since the boundary must be continuous across the ice-liquid boundary. Our choice of $K^{\beta}$ seems to produce the most consistent clathrate stability boundary under the given constraints.

Using the above data and models we calculate $\Delta V^{\beta}$ and write it within each water phase region as linear with pressure. Hence, the form of $\Delta \mu^{\beta}$ is

$$
\Delta \mu^{\beta}(T, P)=\Delta \mu^{\beta}\left(T, P_{0}\right)+c+b P+a P^{2},
$$

where the constants $c, b$, and $a$ apply to the particular water-ice phase stable at temperature $T$ and pressure $P$. 
Calculation of the methane fugacity $f_{c}(T, P)$ differed depending on the temperature because of uneven data coverage and complications in integrating pressure-volume data near the methane critical temperature, $190 \mathrm{~K}$. Figure 9 illustrates the techniques used to evaluate equation (4) on a $P-T$ plot of the pure methane phase diagram. The fugacity at $100 \mathrm{~K}$ is calculated by a direct $P-V$ integration up to the region of interest, found by rough calculation to be $\sim 10$ kilobars, using Goodwin's (1974) data at pressures up to 700 bars and joining that to high-pressure methane-ice data fits constructed by Lupo and Lewis (1980), with liquid-solid phase boundary pressure and $\Delta V$ data of Cheng, Daniels, and Crawford (1975). Since the freezing pressure is 377 bars at $100 \mathrm{~K}$, volume data were available continuously throughout the liquid and solid regimes. At other temperatures,

$$
f_{c}(T, P)=f_{c}\left[T, P_{s}(T)\right] \exp \left\{\frac{1}{k T}\left[-\int_{T}^{100 \mathrm{~K}} S\left(T^{\prime}, P_{s}\right) d T^{\prime}+\int_{P_{s}(T)}^{P} V\left(100 \mathrm{~K}, P^{\prime}\right) d P^{\prime}-\int_{100 \mathrm{~K}}^{T} S\left(T^{\prime}, P\right) d T^{\prime}\right]\right\},
$$

where $P_{s}(T)=$ vapor pressure (or extrapolated vapor pressure) at $T$; see Figure 9. Normally the calculation was done to two pressures, 5 and 10 kilobars, and then a linear fit of $\log f_{c}$ to $P$ constructed for convenience, since the Lupo and Lewis equation-of-state fit is good to only $\sim 8$ kilobars. This approximation turns out to be accurate if the resulting dissociation pressure is not too far above 10 kilobars, as is the case.

Low-pressure entropy was evaluated using a linear fit from Goodwin (1974). Entropy at the high-pressure end was calculated at each temperature $T$ by

$$
S(T, V)=\left.\int_{V_{0}}^{V} \frac{d P}{d T}\right|_{V} d V+S\left(T, V_{0}\right) .
$$

The quantity $S\left(T, V_{0}\right)$ was read off from Goodwin (1974) at 700 bars in the liquid-methane field along with the corresponding volume $V_{0} ; d P /\left.d T\right|_{V}$ was then derived from the same source, and in the absence of additional data was assumed constant up to the liquid-solid boundary pressure. Here the entropy change of freezing from Cheng, Daniels, and Crawford (1975) was added, and then the volume integration continued in the solid field using the empirical fit of Lupo and Lewis (1980). The integration was stopped at 10 kilobars at a volume estimated from the Cheng freezing-curve data, assuming a negligible coefficient of thermal expansion. Since the additional entropy contribution in the solid field amounted to only a few percent of the total, the small error introduced by estimating the final volume was acceptable.

Because of the uncertainties inherent in the above technique, it was desirable to calculate the entropy difference between $T$ and $100 \mathrm{~K}$ at high pressure in an independent fashion. Here $S$ was calculated using the specific heat at constant volume of the methane ice:

$$
S(T, P)-S(100 \mathrm{~K}, P)=\int_{100}^{T} C_{v}\left(T^{\prime}, P\right) \frac{d T^{\prime}}{T^{\prime}},
$$

where the Debye model for solids yields (Reif 1965, p. 415)

$$
C_{v}=C_{v}^{\text {lattice }}+C_{v}^{\text {rotational }}=x 3 k+\frac{3}{2} k,
$$

where the rotational but not vibrational states of the methane molecule are excited at the relevant temperatures (Kerley 1980). The parameter $x$ in equation (A7) is less than unity since the estimated Debye temperature for methane is in excess of $100 \mathrm{~K}$; we estimate $x \sim 0.8-0.9$. The Debye model yields $\Delta S$ from equation (A6), for $T$ in the range 100-230 K, that is generally within $10 \%$ of the value derived above from thermodynamic quantities. The agreement is adequate for our purposes. We then apply a linear fit to $S$ between $T$ and $100 \mathrm{~K}$ to calculate $f_{c}(T, P)$ via equation (A4).

As a check, using available volume data at $180 \mathrm{~K}$ from the above sources, we integrated along an isothermal path to compare the resulting fugacity at 10 kilobars with that derived from the above techniques. The two agree to within $35 \%$, which is acceptable considering the uncertainties in the thermodymamic quantities derived above.

At $T \geqslant 300 \mathrm{~K}$ data from Robertson and Babb (1969) on fluid methane volumes were available to find $f_{c}(T, P)$ by isothermal $V(P)$ integration. Their data were presented from 2 to 10 kilobars at 308,373 , and $473 \mathrm{~K}$, and were fitted to an isothermal linear function of pressure at 3 and 10 kilobars. At other temperatures, thermal expansion coefficients derived from the data were used to interpolate or extrapolate the volume data to the new temperature. Data in Goodwin (1974) to 700 bars were likewise linearly fitted, and a linear interpolation was made between 0.7 and 2 kilobars. Numerical integration by discrete Simpson's approximation of the volume data available showed that the linear fits overestimate the $\int V d P$ integral by $11 \%$ from 300 to 700 bars and by $2 \%$ from 2000 to 10,000 bars. The linear fits to volume were thus adjusted slightly to produce a better fit. The fugacities were calculated by equation (4) with $P_{0}=300$ bars, at which $\phi \sim 1$. (In this supercritical regime extrapolations from the vapor pressure curve cannot be made accurately; we pick a convenient pressure for which $\phi \sim 1$ to begin the integration.) Fugacity calculations by Saito, Marshall, and Kobayashi (1964) at moderate pressures indicate $\phi(300 \mathrm{~K}, 300$ bars $) \sim 0.7$, for which our calculation of dissociation pressures at $P<4$ kilobars fits the Marshall, Saito, and Kobayashi (1964) data more closely than for $\phi=1$. At the high-pressure end ( $>10$ kilobars) the two different values of $\phi$ alter the derived dissociation pressure by $\leqslant 20 \%$; we use $\phi \sim 0.7$ for consistency. 
The form of the methane fugacity at $T>273 \mathrm{~K}$ was expressed as

$$
f_{c}=0.7 \times 300 \text { bars } \times \exp \frac{1}{k T}\left(D+E P+F P^{2}\right),
$$

with $D, E$, and $F$ derived from Goodwin (1974) and Robertson and Babb (1969), different in the intervals $P=300-700$ bars, $P=700-2000$ bars, $P>2000$ bars. As a check, the Robertson and Babb volume data were extrapolated down to $220 \mathrm{~K}$, and $f_{c}$ was calculated at 10 and 12 kilobars and compared with the values calculated using equation (A4). The fugacities disagree by $40 \%$ at 10 kilobars, and $20 \%$ at 12 kilobars. Since the two techniques primarily use different data sets, this agreement gives confidence in both data and techniques.

\section{REFERENCES}

Aaldijk, L. 1971, dissertation, Woensdag, Rotterdam.

Anders, E., and Ebihara, M. 1982, Geochim. Cosmochim. Acta, 46, 2363.

Atreya, S. K., Donahue, T. M., and Kuhn, W. R. 1978, Science, 201, 611.

Avnir, D., Farin, D., and Pfeifer, P. 1984, Nature, 308, 261.

Barrer, R. M. 1941, Diffusion in and through Solids (Cambridge: University Press)

1967, in The Solid-Gas Interface, Vol. 2, ed. E. A. Flood (New York: Marcel Dekker), p. 557.

Barrer, R. M., and Edge, A. V. J. 1967, Proc. Roy. Soc. London, A, $300,1$.

Barrer, R. M., and Ruzicka, D. J. 1962, Trans. Faraday Soc., 58, 2262.

Bertie, J. E., and Jacobs, S. M. 1977, Canadian J. Chem., 55, 1777. 1978, J. Chem. Phys., 69, 4105. 1982, J. Chem. Phys., 77, 3230.

Bridgeman, P. 1913, Proc. Am. Acad. Arts and Sciences, 48, 307. 1937, J. Chem. Phys., 5, 964.

Brownlee, D. E. 1978, in IAU Colloquium 52, Protostars and Planets, ed. T. Gehrels (Tucson: University of Arizona Press), p. 134.

Byk, S. Sh., and Fomina, V. I. 1968, Russian Chem. Rev., 37, 469.

Carman, P. C. 1956, Flow of Gases through Porous Media (New York: Academic Press).

Carslaw, H. S., and Jaeger, J. C. 1959, Conduction of Heat in Solids (Oxford: Clarendon Press)

Chan, J. P., and Giauque, W. F. 1964, J. Phys. Chem., 68, 3053.

Cheng, A. F., and Lanzerotti, L. J. 1978, J. Geophys. Res., 83, 2596.

Cheng, V. M., Daniels, W. B., and Crawford, R. K. 1975, Phys. Rev. B, 11, 3972.

Claypool, G. E., and Kvenvolden, K. A. 1983, Ann. Rev. Earth Planet. Sci., 11, 299.

Clifford, I. L., and Hunter, E. 1933, J. Phys. Chem., 37, 101

Cook, J. G., and Leaist, D. G. 1983, Geophys. Res. Letters, 10, 397.

CRC Handbook of Chemistry and Physics 1970, ed. R. C. Weast (Cleve-

land: Chemical Rubber Company)

Culberson, O. L. 1951, Petrol. Trans. AIME, 192, 223.

Davidson, D. W. 1971, Canadian J. Chem., 49, 1224. 1973, in Water: $A$ Comprehensive Treatise, Vol. 2, ed. F. Franks

(New York: Plenum), p. 115.

Davidson, D. W., Handa, Y. P., Ratcliffe, C. I., Tse, J. S., and Powell, B. M. 1984, Nature, 311, 142.

Davidson, D. W., and Wilson, G. J. 1963, Canadian J. Chem., 41, 1424

Delsemme, A. H. 1983, in Comets, ed. L. Wilkening (Tucson: University of Arizona Press), p. 85

Delsemme, A. H., and Miller, D. C. 1970, Planet. Space Sci., 18, 717

Delsemme, A. H., and Swings, P. 1952, Ann. d'Ap., 15, 1.

Delsemme, A. H., and Wenger, A. 1970, Planet. Space Sci., 18, 709.

Dharma-Wardana, M. W. C. 1983, J. Phys. Chem., 87, 4185.

Diepen, G. A. M., and Scheffer, F. E. C. 1950, Rec. Trav. Chim., 69, 593.

Donahue, T. M., and Pollack, J. B. 1983, in Venus, ed. D. M. Hunten, L.

Colin, T. M. Donahue, and V. I. Moroz (Tucson: University of Arizona Press), p. 1003.

Dorsey, N. E. 1940, Properties of Ordinary Water Substance (Scranton, Penn.: Reinhold)

Eisenberg, D., and Kauzmann, W. 1969, The Structure and Properties of Water (New York: Oxford University Press)

Ellsworth, K., and Schubert, G. 1983, Icarus, 54, 490.

Evans, R. B., III, Watson, G. M., and Mason, E. A. 1961, J. Chem. Phys., 35, 2076.

Fanale, F. P., Banerdt, W. B., Saunders, R. S., Johansen, L. A., and Salvail, J. R. 1982, J. Geophys. Res., 87, 10215.

Fink, U., and Sill, G. 1982, in Comets, ed. L. Wilkening (Tucson: University of Arizona Press), p. 164

Fowler, R., and Guggenheim, E. A. 1960, Statistical Thermodynamics (Cambridge: Cambridge University Press).

Friedson, A. J., and Stevenson, D. J. 1983, Icarus, 56, 1.
Gaffney, E. S., and Matson, D. L. 1980, Icarus, 44, 511.

Gautier, D., Bezard, B., Marten, A., Baluteau, J. P., Scott, N., Chedin, A., Kunde, V., and Hanel, R. 1982, Ap. J., 257, 901.

Giauque, W. F., and Stout, J. W. 1936, J. Am. Chem. Soc., 58, 1144.

Goodwin, R. D. 1974, NBS Tech. Note 653, (Washington, D.C.: US Government Printing Office).

Grace, J. D., and Kennedy, G. C. 1967, J. Phys. Chem. Solids, 28, 977.

Greenberg, M. 1983, in Comets, ed. L. Wilkening (Tucson: University of Arizona Press), p. 131

Gurikov, Y. B. 1983, Zh. Fiz. Khim., 57, 439

Hagan, M. M. 1962, Clathrate Inclusion Compounds (New York: Reinhold).

Haltenhorth, H., and Klinger, J. 1969, in Physics of Ice: Proceedings of International Symposium on Physics of Ice, ed. N. Riehl, B. Bullemer, and H. Engelhardt (New York: Plenum Press), p. 579.

Hartmann, W. K. 1973, Icarus, 18, 634.

Haudenschild, C. 1970, JPL Space Programs Sum. III, 4.

Hildenbrand, D. L., and Giauque, W. F. 1953, J. Am. Chem. Soc., 75, 2811.

Hirschfelder, J. O., Curtiss, C. F., and Bird, R. B. 1954, Molecular Theory of Gases and Liquids (New York: John Wiley).

Hobbs, P. V. 1974, Ice Physics (Oxford: Clarendon Press)

Holder, G. D., Corbin, G., and Papadopoulos, K. D. 1980, Industrial Eng. Chem., Fund., 19, 282.

Hunten, D. M. 1978, in The Saturn System, ed. D. M. Hunten and D. Morrison (NASA CP 2068), p. 127.

Hunten, D. M., Flasar, F. M., Samuelson, R. E., Strobel, D. F., and Stevenson, D. J. 1984, in Saturn, ed. T. Gehrels (Tucson: University of Arizona Press), p. 671.

International Critical Tables, Vol. 3. 1982, (New York: McGraw-Hill).

Jacobsen, R. T., and Stewart, R. B. 1973, J. Phys. Chem. Ref. Data, 2, 757.

Jeffrey, G. A., and McMullan, R. K. 1967, Progr. Inorg. Chem., 8, 43.

Johari, G. P., and Chew, H. A. M. 1984, Philosophical Mag., B, 49, 281-294.

Johnson, M. L., Schwake, A., and Nicol, M. 1985, In Proceedings of NATO Conference, Ices in the Solar System, ed. J. Klinger (in press).

Kerley, G. I. 1980, J. Appl. Phys., 51, 5368.

Korvezee, A. E., and Scheffer, F. E. C. 1931, Rec. Trav. Chim., 50, 256.

Kvenvolden, K. A., and McDonald, T. J. 1982, AGU Trans. EOS, 63 , 1015.

Kvenvolden, K. A., and McMenamin, M. A. 1980, US Geol. Surv. Circ. 825.

Landau, L. D., and Lifshitz, E. M. 1969, Statistical Physics (Oxford: Pergamon Press).

Lewis, J. S. 1969, Icarus, 10, 365.

1971, Icarus, 15, 174.

1974, Science, 186, 440

Lewis, J. S., and Prinn, R. G. 1980, Ap. J., 238, 357

Lindal, G. F., Wood, G. E., Hotz, H. B., Sweetnam, D. N., Eshleman, V. R, and Tyler, G. L. 1983, Icarus, 53, 348.

Lunine, J. I., and Stevenson, D. J. $1982 a$, Bull. AAS, 14, 713. 1982 b, Icarus, 52, 14.

Lunine, J. I., Stevenson, D. J., and Yung, Y. L. 1983, Science, 222, 1229.

Lupo, M. J., and Lewis, J. S. 1979, Icarus, 40, 157. 1980, Icarus, 42, 29.

Lutz, B. L., Owen, T., and Cess, R. D. 1976, Ap. J., 203, 541.

Mandelbrot, B. B. 1977, Fractals: Form, Chance, and Dimension (San Francisco: W. H. Freeman).

Marion, J. B. 1970, Classical Dynamics of Particles and Systems (New York: Academic Press).

Marshall, D. R., Saito, S., and Kobayashi, R. 1964, A.I.Ch.E.J., 10, 202.

Mazo, R. M. 1964, Molec. Phys., 8, 515.

McKoy, V., and Sinanoglu, O. 1963, J. Chem. Phys., 38, 2946. 
Mel'nik, Yu. P. 1978, Geochem. Intl., 15 (6), 49

Miller, S. L. 1961, Proc. Nat. Acad. Sci., 47, 1798. 1969, Science, 165, 489.

1973, in Physics and Chemistry of Ice, ed. E. Whalley, S. J. Jones, and L. W. Gold (Ottawa: Royal Society of Canada), p. 42. 1974 , in Natural Gases in Marine Sediments, ed. I. R. Kaplan (New York: Plenum Press), p. 151.

Miller, S. L., and Smythe, W. D. 1970, Science, 170, 531.

Mizuno, H. 1980, Progr. Theor. Phys., 64, 544.

Mukhin, L. M. 1983 , in Venus, ed. D. M. Hunten, L. Colin, T. M. Donahue, and V. I. Moroz (Tucson: University of Arizona Press), p. 1037.

Nagata, I., and Kobayashi, R. 1966, Industrial Eng. Chem., Fund., 5, 344. Omar, M. H., Dokoupil, Z., and Schroten, H. G. M. 1962, Physica, 28, 309 .

Owen, T. 1982, Planet. Space Sci., 30, 833.

Parrish, W. R., and Prausnitz, J. M. 1972, Industrial Eng. Chem., Process. Design Development, 11, 26.

Pauling, L., and Marsh, R. E. 1952, Proc. Nat. Acad. Sci., 38, 112.

Pearson, C. F., Halleck, P. M., McGuire, P. L., Hermes, R., and Mathews, M. 1983, J. Phys. Chem., 87, 4180

Pinder, K. L. 1964, Canadian J. Chem. Eng., 42, 132.

Plummer, P. L. M., and Chen, T. S. 1983, J. Phys. Chem., 87, 4190.

Pollack,'J. B., and Black, D. C. 1982, Icarus, 51, 169.

Pollack, J. B., and Consolmagno, G. 1984, in Saturn, ed. T. Gehrels (Tucson: University of Arizona Press), p. 811.

Prausnitz, J. M. 1969, Molecular Thermodynamics of Fluid-Phase Equilibria (Englewood Cliffs, N.J.: Prentice-Hall)

Prinn, R. G., and Fegley, B., Jr. 1981, Ap. J., 249, 308.

Rebiai, R., Rest, A. J., and Scurlock, R. G. 1983, Nature, 305, 412.

Reif, F. 1965, Fundamentals of Statistical and Thermal Physics (New York: McGraw-Hill).

Robertson, S. L., and Babb, S. E., Jr. 1969, J. Chem. Phys., 51, 1357.

Rollet, A.-P., and Vuillard, G. 1956, Comptes Rendus Acad. Sci., 243, 383.

Ross, R. G., and Andersson, P. 1982, Canadian J. Chem., 60, 881

Saito, S., Marshall, D. R., and Kobayashi, R. 1964, A.I.Ch.E.J., 10, 734.

Samuelson, R. E., Maguire, W. C., Hanel, R. A., Kunde, V. G., Jennings, D. E., Yung, Y. L., and Aikin, A. C. 1983, J. Geophys. Res., 88, 8709.

Selleck, F. T., Carmichael, L. T., and Sage, B. H. 1952, Industrial Eng. Chem., 44, 2219.

Shakespeare, W. 1968, Titus Andronicus, ed. J. D. Wilson (Cambridge: University Press).

JonAThan Lunine: Lunar and Planetary Lab., University of Arizona, Tucson, AZ 85721

David J. Stevenson: 170-25 Caltech, Pasadena, CA 91125
Shipley, T. H., and Didyk, B. M. 1982, Init. Reports Deep Sea Drilling Project, 66, 547.

Shipley, T. H., Houston, M. H., Buffler, R. T., Shaub, F. J., McMillen, K. J., Ladd, J. W., and Worzel, J. L. 1979, Bull. Am. Assoc. Petrol. Geol., 63, 2204.

Shmulovich, K. I., Mazur, V. A., Kalinichev, A. G., and Khodorevskaya, L. I. 1980, Geochem. Internat., 17 (6), 18.

Shoji, H., and Langway, Jr., C. C. 1982, Nature, 298, 548

Sill, G. T., and Wilkening, L. L. 1978, Icarus, 33, 13.

Smythe, W. D. 1975, Icarus, 24, 421

Sortland, L. D., and Robinson, D. B. 1964, Canadian J. Chem. Eng., 42 (1), 38 .

Stevenson, D. J. $1982 a$, Nature, 298, 142

.1982 b, Lunar Planet. Sci. Conf. Abstract XIII, p. 770.

1984, paper presented at Uranus Neptune Workshop, Pasadena, California, February 6-8.

Stoll, R. D., and Bryan, G. M. 1979, J. Geophys. Res., 84, 1629.

Strobel, D. F., and Shemansky, D. E. 1982, J. Geophys. Res., 87, 1361

Tammann, G., and Krige, G. J. R. 1925, Zs. Anorganische und Allegemeine Chemie, 146, 179.

Tester, J. W., Bivins, R. L., and Herrick, C. C. 1972, A.I.Ch.E.J., 18, 1220.

Tse, J. S., Klein, M. L., and McDonald, I. R. 1983, J. Phys. Chem., 87, 4198.

Tsiklis, D. S., Linshits, L. R., and Goryunova, N. P. 1965, Russian J. Phys. Chem., 39, 1590.

van Berkum, J. G., and Diepen, G. A. M. 1979, J. Chem. Thermodyn., 11 317

van Cleef, A., and Diepen, G. 1965, Rec. Trav. Chim., 84, 1085.

van der Waals, J. H. 1956, Trans. Faraday Soc., 52, 184.

van der Waals, J. H., and Platteeuw, J. C. 1959, Adv. Chem. Phys., 2, 1

van Kasteren, P. H. G. 1973, Bull. Inst. Int. Froid Annexe, 4, 81.

von Stackelberg, M., and Meinhold, W. 1954, Zs. Elektrochemie, 58, 40

von Stackelberg, M., and Muller, H. R. 1954, Zs. Elektrochemie, 58, 25.

Watson, A. J., Donahue, T. M., and Walker, J. C. G. 1981, Icarus, 48 , 150.

Weidenschilling, S. J. 1977, M.N.R.A.S., 180, 57.

Whalley, E. 1980, J. Geophys. Res. 85, 2539.

Weibe, R. and Gaddy, V. L. 1937, J. Am. Chem. Soc. 59, 1984.

Yung, Y. L., Allen, M., and Pinto, J. 1984, Ap. J. Suppl., 55, 465. 